\title{
New Concepts for STED Microscopy
}

\section{Dissertation}

\author{
for the award of the degree \\ „Doctor rerum naturalium" \\ of the Georg-August-Universität Göttingen
}

within the doctoral program physics

of the Georg-August University School of Science (GAUSS)

submitted by

\section{Julia Kratz}

from Gießen 


\section{Thesis Committee:}

apl. Prof. Dr. Alexander Egner

Optische Nanoskopie

Institut für Nanophotonik Göttingen e.V.

Prof. Dr. Jörg Enderlein

III. Physikalisches Institut

Georg-August-Universität Göttingen

Prof. Dr. Sarah Köster

Institut für Röntgenphysik

Georg-August-Universität Göttingen

\section{Members of the Examination Board:}

Reviewer:

Second Reviewer: apl. Prof. Dr. Alexander Egner

Prof. Dr. Jörg Enderlein

\section{Further Members of the Examination Board:}

Prof. Dr. Sarah Köster

Institut für Röntgenphysik

Georg-August-Universität Göttingen

Prof. Dr. Andreas Janshoff

Institut für Physikalische Chemie

Georg-August-Universität Göttingen

apl. Prof. Dr. Michael Seibt

IV. Physikalisches Institut

Georg-August-Universität Göttingen

Prof. Dr. Stefan Klumpp

Institut für Dynamik komplexer Systeme

Georg-August-Universität Göttingen

Date of the oral examination: 25 October 2021 


\section{Abstract}

Fluorescence nanoscopy allows to non-invasively resolve three-dimensional cellular structures beyond the diffraction limit [41]. One of these high resolution imaging techniques is stimulated emission depletion (STED) microscopy [44]. However, the practically achievable resolution of a STED microscope is often limited by photobleaching [39]. One method to overcome this limitation is tomographic STED (tomoSTED) microscopy [60]. In tomoSTED microscopy, excited fluorophores in the sample plane are depleted by 1D STED patterns, which lead to an effective narrowing of the fluorescence-allowed area in a single direction. As the effective 1D STED-PSFs exhibit both a higher resolution in the respective direction as well as a higher signal as compared to conventional STED, the STED laser power as well as the exposure time can be reduced, leading to a lower light dose. A highly resolved image in two dimensions is reconstructed from multiple images, each exhibiting a different orientation of the 1D STED-PSF. The number of required pattern orientations depends in this context on the ratio of the resolutions in the depleted and non-depleted direction. Since the resolution per pattern orientation is only increased along a single direction, imaging along the other direction is still diffractionlimited. Therefore, the resolution along this direction can be increased by utilizing the concept of image scanning microscopy (ISM) and the number of required pattern orientations can be accordingly reduced. This leads to a lower overall acquisition time and translates directly into a lower light dose. Within this thesis, the combination of tomoSTED and ISM was investigated for the first time, both in theory and experiment. Furthermore, it was investigated whether the tomoSTED principle can be extended from $2 \mathrm{D}$ to $3 \mathrm{D}$. 



\section{Contents}

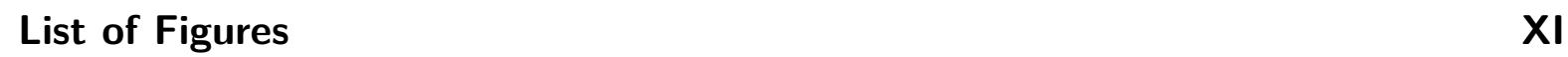

\begin{tabular}{lll}
\hline Abbreviations & XII
\end{tabular}

$\begin{array}{ll}\text { 1. Motivation and Objectives } & 1\end{array}$

2. Theoretical Framework 4

2.1. Confocal scanning fluorescence microscopy . . . . . . . . . . . . . . . . . 4

2.1.1. Basics of fluorescence . . . . . . . . . . . . . . . . . 4

2.1.2. Image formation in confocal scanning fluorescence microscopy . . 6

2.2. STED microscopy . . . . . . . . . . . . . . . . . . . . . 9 9

2.3. Two-dimensional tomographic STED microscopy . . . . . . . . . . . . . . 14

3. Material and Methods 19

3.1. Experimental realization . . . . . . . . . . . . . . . . . . . . . . . . . 19

3.2. Polarisation adjustment using liquid crystal retarders . . . . . . . . . . . 22

3.2.1. Liquid crystal retarders . . . . . . . . . . . . . . . . . . . . . . . . . . . . . . . . 22

3.2.2. Description of the polarisation states . . . . . . . . . . . . . 23

3.2.3. Generation of linear polarised light with liquid crystal retarders . 25

3.2.4. Liquid crystal retarder response time . . . . . . . . . . . . . . 27

3.3. Reconstruction of tomoSTED images . . . . . . . . . . . . . . . 30

3.3.1. Maximum-value reconstruction . . . . . . . . . . . . . . . . 30

3.3.2. Richardson-Lucy deconvolution . . . . . . . . . . . . . . . . 31

3.3.2.1. Estimation of the underlying object . . . . . . . . . . . 32

3.3.2.2. Estimation of the underlying object in tomoSTED . . . 34

3.3.2.3. Limitations of the deconvolution algorithm. . . . . . . . 34

3.3.2.4. Similarity quantification in the case of a known object . 35

4. ISM-assisted tomographic STED microscopy 37

4.1. Image formation in image scanning microscopy . . . . . . . . . . . . . . . . 39

4.2. Appliance of ISM to tomoSTED microscopy . . . . . . . . . . . . . . . . 43

4.2.1. Realization of ISM-assisted tomoSTED microscopy . . . . . . . . 43

4.2.2. Theoretical consideration for reassigning ISM-tomoSTED images. 44

4.2.3. Practical realization of the reassignment of ISM-tomoSTED images 45

4.2.4. Validation of the pixel reassignment of ISM-tomoSTED via simu-

lated data . . . . . . . . . . . . . . . . . . . . . 48 
4.2.5. Experimental validation of the appliance of ISM to tomoSTED. . 51

4.2.6. Calibration of the ISM microscope . . . . . . . . . . . . 53

4.3. Number of pattern orientations for ISM-tomoSTED . . . . . . . . . . . . 55

4.3.1. Determination of the number of pattern orientations for ISM-tomoSTED in Fourier space . . . . . . . . . . . . . . . . . . . 55

4.3.1.1. Theoretical derivation of the number of pattern orientations for ISM-tomoSTED . . . . . . . . . . . . . . 56

4.3.1.2. Validation of the theoretical derivation via simulated and experimental data . . . . . . . . . . . 60 60

4.3.2. Validation of the number of pattern orientations in real space via simulations with a ring-shaped object . . . . . . . . . . . 63

4.4. Validation of ISM-tomoSTED on experimental data . . . . . . . . . . 64

5. Three-dimensional tomographic STED microscopy 67

5.1. Phase masks for the rotation in three dimensions . . . . . . . . . . 67

5.1.1. Intensity distribution within the focal region for high numerical aperture objective lenses . . . . . . . . . . . . . . . . . . . 68

5.1.2. Derivation of phase masks for the rotation in y-z direction . . . . 71

5.1.3. Validation of the phase masks for the rotation in $\mathrm{y}-\mathrm{z}$ direction on simulated data . . . . . . . . . . . . . 76

5.2. Reduction factor for the laser power . . . . . . . . . . . . . . . . . 81

5.3. Reduction factor for the exposure time . . . . . . . . . . . . . . 82

5.4. Selection of 3D tomoSTED orientations . . . . . . . . . . . . . 85

5.5. 3D tomoSTED using the maximum-value reconstruction method . . . . . 89

5.6. 3D tomoSTED using Richardson-Lucy deconvolution . . . . . . . . . . . 93

5.6.1. Characterisation of the performance of classical 3D STED . . . . 94

5.6.2. Characterisation of the performance of 3D tomoSTED . . . . . . 96

$\begin{array}{ll}\text { 6. Conclusion and Outlook } & 99\end{array}$

\begin{tabular}{ll}
\hline References & 102
\end{tabular}

\begin{tabular}{ll}
\hline Appendices & 112
\end{tabular}

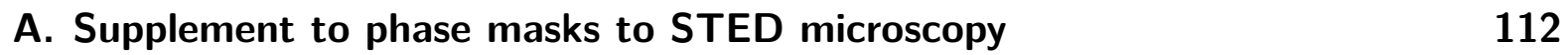

B. Supplement to validation of the pixel reassignment of ISM-tomoSTED via simulated data 



\section{List of Figures}

2.1. Jablonski diagram . . . . . . . . . . . . . . . . . . . 4

2.2. Illustration of the imaging of objects through a microscope . . . . . . . 7

2.3. Illustration of the two required distinct optical molecule states for RESOLFT microscopy . . . . . . . . . . . . . . . . . . . . . 9

2.4. Principle of STED microscopy . . . . . . . . . . . . . . . . . . . . . 11

2.5. Comparison of $1 \mathrm{D}$ and $2 \mathrm{D}$ depletion pattern . . . . . . . . . . . . 15

2.6. Illustration of the tomoSTED principle . . . . . . . . . . . . . . 18

3.1. Schematic illustration of the experimental setup . . . . . . . . . . . . . 19

3.2. Illustration of polarisation states . . . . . . . . . . . . . . . . 24

3.3. Adjusting of linear polarisation using a combination of two LCRs . . . . 26

3.4. Schematic illustration of the LCR response time measurement setup . . . 28

3.5. Exemplary LCR's response time measurement . . . . . . . . . . . . . . . 29

4.1. Schematic illustration of the idea using ISM to reduce the number of depletion pattern orientations of tomoSTED . . . . . . . . . . . . 37

4.2. Illustration of scanning with a detector array . . . . . . . . . . . . . 39

4.3. Illustration of the ISM-assisted tomoSTED microscopy operation principle 44

4.4. Validation of the phase correlation method for the pixel reassignment of

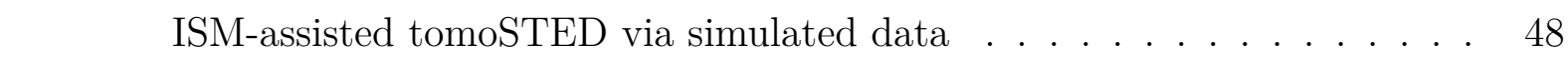

4.5. Investigation of the dependence of the scan pixel size and the pixel size of the detector array on the resolution of the ISM PSF . . . . . . . . . . . . 50

4.6. Examples of experimentally recorded microscope PSFs (measured on $48 \mathrm{~nm}$

\begin{tabular}{|c|}
\hline fluorescent (crimson) microspheres) for different orientations of the high \\
\hline
\end{tabular}

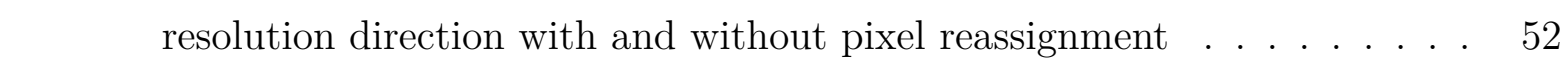

4.7. Calibration of the pixel size and the width of the detection PSF . . . . . 54

4.8. Schematic illustration of the theoretical derivation of the number of pattern orientations for ISM-tomoSTED . . . . . . . . . . . . . . . 56

4.9. Theoretically derived number of pattern orientations for ISM-tomoSTED in dependence on the number of pattern orientations of tomoSTED . . . 59

4.10. Analysis of tomoSTED as well as ISM-tomoSTED OTFs. . . . . . . . . . 62

4.11. Validation of the number of pattern orientation for ISM-tomoSTED in

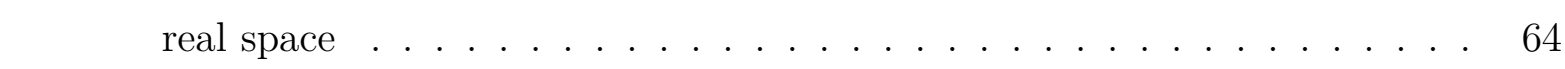

4.12. Experimental validation of ISM-tomoSTED with a reduced number of pattern orientations . . . . . . . . . . . . . . 6 65 
5.1. Schematic illustration of the focussing process of a microscopes objective lens with high numerical aperture . . . . . . . . . . . . . . . . 69

5.2. Decomposition of the electric field's amplitude for the case of incoming x-polarised light . . . . . . . . . . . . . . . . . . . . . . . . . . . 73

5.3. Illustration of the factor $d(\beta, \theta, \phi)$ in dependence on the angle $\beta$. . . . 74

5.4. Maximum intensity of the effective PSF as a function of the quantile employed for the $\beta=70^{\circ}$ 3D tomoSTED phase mask . . . . . . . . . 76

5.5. Phase masks for 3D tomoSTED and simulated 3D tomoSTED STED intensity distributions . . . . . . . . . . . . . . . . . . 77

5.6. Angle of the maxima's position in relation to the rotation angle $\beta$. . . . 78

5.7. Experimentally measured STED intensity distributions using the 3D to-

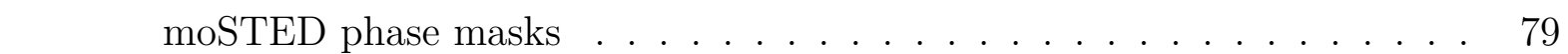

5.8. Simulated 3D tomoSTED effective PSFs in relation to the rotation angle $\beta 80$

5.9. Maximum reduction factor of the laser power in relation to the individual 3D tomoSTED PSFs . . . . . . . . . . . . . . . . 82

5.10. Exemplary illustration of single 3D tomoSTED effective PSFs' background

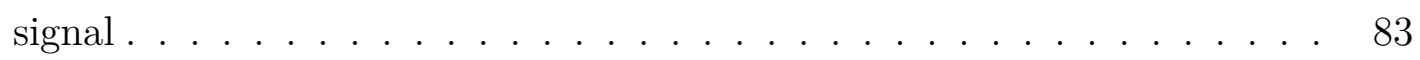

5.11. Exposure time scaling factor in relation to the rotation angle $\beta$. . . . . . 84

5.12. Illustration of the rotation of the individual 3D tomoSTED PSFs in three

- dimensions . . . . . . . . . . . . . . . . . . . 86

5.13. Illustration of the selection of 3D tomoSTED orientations for reconstruction 88

5.14. Comparison of final 3D tomoSTED PSFs reconstructed using the maximum-

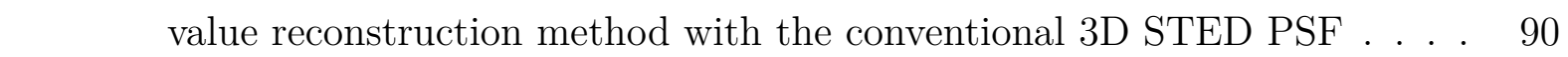

5.15. Resolution of 3D tomoSTED arrangement with 19 orientations as a func-

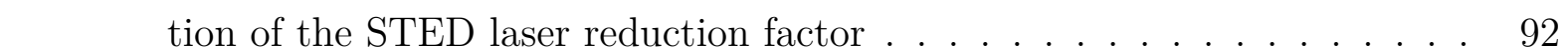

5.16. Investigation of the classical 3D STED via the Richardson-Lucy deconvo-

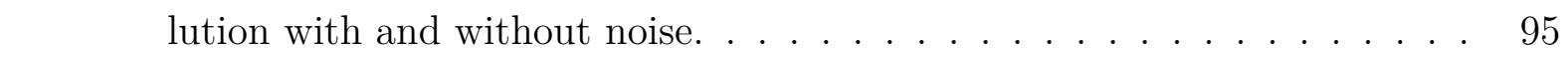

5.17. Illustration of the object identified as correctly recoverable for 3D STED

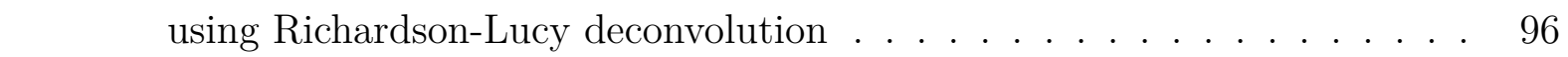

5.18. Estimation of the object identified by 3D STED for 3D tomoSTED imag-

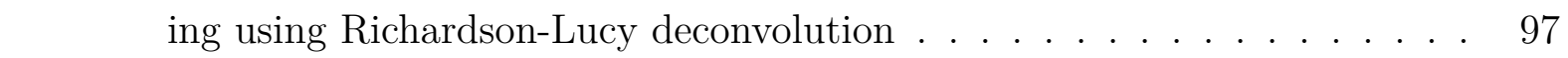

A.1. Comparison of the steepness for the phase masks in $\mathrm{x}-\mathrm{y}$ and z-direction . 113

B.1. Comparison of $45^{\circ}$ tomoSTED and ISM-tomoSTED PSF . . . . . . . . . 114

C.1. Comparison of the resolution in $\mathrm{x}$-and z-direction of final 3D tomoSTED

PSFs reconstructed via the maximum-value reconstruction with the reso-

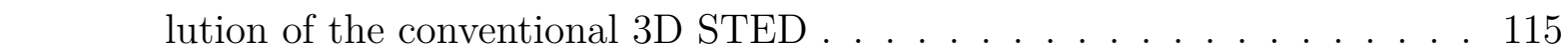


C.2. Resolution of 3D tomoSTED arrangement with 9 orientations as a function of the STED laser reduction factor . . . . . . . . . . . . . . . . . . . . . 115 


\section{Abbreviations}

1D, 2D, 3D

APD

$\mathrm{BF}$

BS

conf

det

DM

DyMIN

exc

$\mathrm{f}$

FWHM

FRET

GT

ISM

HWP

LCR

$\max$

MSE

MOST

OTF

$\mathrm{PSF}$

QWP

RESCUE

RESOLFT

SCMOS

STED

SNR

SLM

tomoSTED

T-Rex one-,two-,three-dimensional

avalanched photodiode

emission bandpass filter

pellicle beam splitter

confocal

detection

dichroic mirror

dynamic intensity minimum

excitation

focal length

full width at half maximum

Förster resonance energy transfer

Glan Thompson prism

image scanning microscopy

half waveplate

liquid crystal retarder

maximum

mean square error

multiple off-state transitions

optical transfer function

point spread function

quarter wave plate

reduction of state transition cycles

reversible saturable optical fluorescence transitions

scientific complementary metal-oxide-semiconductor

stimulated emission depletion

signal to noise ratio

spatial light modulator

tomographic STED microscopy

triplet relaxation 


\section{Motivation and Objectives}

Fluorescence microscopy has become the most widely used imaging technique in biological science [31, 32, 64]. It enables to image the three-dimensional internal structure of cells or organisms [30]. In addition, biological samples cannot only be studied, but also observed non-invasively, as the method is based on light [41, 78. This allows to investigate cells or organisms in vivo as well as in their native environments [30]. A further advantage of fluorescence microscopy is the visualization of cellular components with high specificity [31, 78]. Through molecular labelling, cell components can be made visible in a targeted manner [31, 78]. By using multiple colours, different components within cells or organisms can be identified and imaged simultaneously [64, 78].

However, the ability of a fluorescence microscope to distinguish individual objects with same colour is fundamentally limited by the wavelength of the used light [1, 30]. If two objects are too close, their images cannot be discerned and thus they can no longer be identified as individual objects. Hence, they cannot be resolved [39, 42, 78] and detailed information about their spatial organization is not obtainable. For visible light, the minimum distance at which two objects can be resolved is approximately $200 \mathrm{~nm}$ in the lateral and about $450 \mathrm{~nm}$ in the axial direction [41]. To be able to investigate cellular components such as proteins, nucleic acids or lipids at the subcellular level [41, 79], highresolution fluorescence microscopy techniques have been developed which circumvent this diffraction limit [42]. One of these fluorescence nanoscopy techniques is stimulated emission depletion (STED) microscopy [44, 53].

In STED microscopy [33, the fluorescent area in the sample plane is narrowed by a targeted deactivation of excited molecules. To this end, the sample's fluorophores are typically illuminated with a doughnut-shaped STED focus shortly after their excitation by a focused excitation beam. For sufficiently high focal STED intensities, the STED light efficiently deactivates the excited fluorophores via the process of stimulated emission. Due to the doughnut-shape of the STED focus, only the molecules located in its centre remain in the excited state and are able to emit fluorescence. In this way, the fluorescent area is spatially confined in two dimensions, which is synonymous with a resolution improvement. The confinement is inversely proportional to the applied STED-intensity $\left(\propto 1 / \sqrt{I_{\text {STED }}}\right)$ [33, 39] and can therefore theoretically become infinitely small. In practice, however, the achievable resolution is mainly limited by the quality of the doughnut's central minimum on the one hand, and by the photostability of the 
fluorophores on the other hand [22, 39]. Thus, too high STED intensities can lead to photobleaching of the sample. So far multiple strategies have been developed to overcome this drawback. For example, new photostable dyes were designed [108]. The use of longer STED-pulses ranging from $150 \mathrm{ps}$ to half the applied fluorophore's lifetime [22] as well as the employment of low repetition-rate lasers (T-Rex) or alternatively fast scanners [20] have been suggested. Furthermore, more sample-protecting illumination schemes have been proposed like the concept of MOST nanoscopy [17] in which the fluorophores, not wanted to be observed, are targeted deactivated before illuminating the sample with the excitation and STED- beam or RESCue [95], in which the sample illumination is switched off if no fluorophores are present at the scan position or if a sufficiently high signal-to-noise ratio has been achieved. In addition, MINFIELD [27], which limits the scan field to the size of the doughnut valley, and DyMIN [35] as a combination of RESCue and MINFIELD have been presented.

Another sample-preserving microscopy method, that was developed for STED imaging with a reduced light dose, is tomoSTED microscopy [59, 60]. Unlike the other methods mentioned above, tomoSTED microscopy [59, 60] uses a modification of the depletion pattern. Contrary to the conventional doughnut shape, it employs a one dimensional depletion pattern. With this, the fluorescent area in the sample plane is only narrowed in one dimension and not in two dimensions as in the conventional variant. The use of the one dimensional depletion pattern leads to a higher resolution in the respective direction as well as to a higher recorded signal as compared to the conventional variant. Therefore, the STED laser power as well as the exposure time can be reduced resulting in a lower light dose. The full high-resolution spatial information of the object in two dimensions is obtained by rotating the depletion pattern. The final image is reconstructed from multiple images, each recorded with a different orientation of the depletion pattern. The number of required depletion pattern orientations depends on the ratio of the resolutions in the depleted and not-depleted direction [59, 60]. Consequently, the number of depletion pattern orientations could be reduced if it is possible to increase the resolution in the not-depleted, i.e. the diffraction-limited, direction. A reduced number of pattern orientations leads to a shorter overall acquisition time which directly translates into a lower light dose applied to the sample. Therefore, by reducing the number of pattern orientations, a more sample-gentle imaging technique can be obtained. Image scanning microscopy is known to improve the resolution of a confocal microscope (with open pinhole) by a factor of $\sqrt{2}$ while maintaining the signal [13, 70]. Therefore, the idea was to combine tomoSTED microscopy with image scanning microscopy to increase the 
resolution in the diffraction-limited direction. Thus, one objective of this thesis was the combination of image scanning microscopy with tomoSTED microscopy (see section 4).

Until now, tomoSTED microscopy has only been developed for high resolution imaging in two dimensions. In this implementation, the resolution along the optical axis remains unaffected and is more than twice as low as compared to the resolution in the lateral plane without the employment of a STED beam. However, most biological samples, such as cells, are three-dimensional. Thus, the two-dimensional tomoSTED microscopy has to be extended into a three-dimensional imaging modality. A further aim of this thesis was therefore the development of three-dimensional tomoSTED microscopy (see section 5). 


\section{Theoretical Framework}

\subsection{Confocal scanning fluorescence microscopy}

The microscopy techniques, used within this thesis, are based on confocal scanning fluorescence microscopy. Therefore, the phenomenon of fluorescence is described in section 2.1.1, as well as processes competing to fluorescence. Additionally, the image formation in confocal fluorescence microscopy is considered in section 2.1 .2 .

\subsubsection{Basics of fluorescence}

Fluorescence is the spontaneous emission of light occurring within nanoseconds after the absorption of light [64]. Schematically, the process of fluorescence and other potential de-excitation processes which might happen after excitation of a molecule are presented in the Jablonski diagram in figure 2.1.

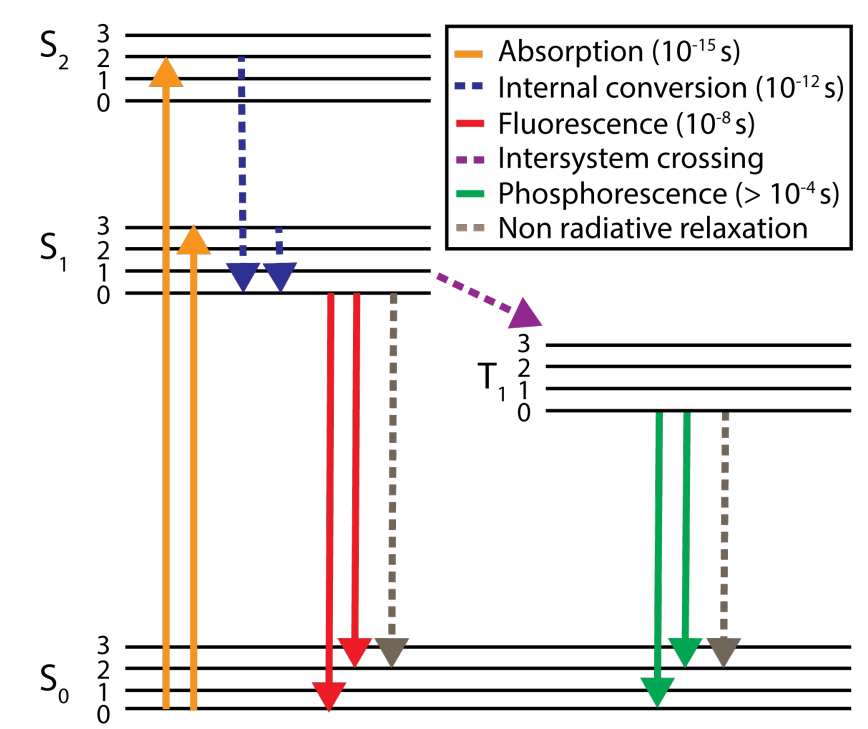

Figure 2.1: Jablonski diagram, modified from 64]

Usually, the potential excitation states of molecules are distinguished between electronically excited states, vibrational excited states and rotational excited states. In figure 2.1. the potential electronic states are marked with $\mathrm{S}_{0}, \mathrm{~S}_{1}, \mathrm{~S}_{2}$ and $\mathrm{T}_{1}$ and the vibrational states with numbered horizontal lines. Several rotational states are related to each vibrational state, but are skipped in figure 2.1. The letters $\mathrm{S}$ and $\mathrm{T}$ indicate the distinction of the states into singlet states $\mathrm{S}$ and triplet states $\mathrm{T}$. The ground state of common fluorescent molecules is a singlet state. That means that the electrons are all paired with 
opposite spins in the molecule orbitals of the ground state. Since the excitation of an electron happens under spin conservation, the molecule passes over in an excited singlet state after absorption of a photon with a suitable wavelength. This transition occurs on a fast time scale $\left(10^{-15} \mathrm{~s}\right)$ and is marked in figure 2.1 with a orange arrow. Most commonly, the excited electron then relaxes, e.g. due to collisions with other molecules, to the lowest vibrational level of the first excited singlet state $S_{1}$. These relaxation processes are called internal conversion and vibrational relaxation and occur on a time scale of $10^{-14}-10^{-11} \mathrm{~s}$ (marked in blue). From the lowest vibrational level of $S_{1}$, there are several possibilities for returning to the ground state. The electron can be de-excited radiationless (e.g. due to quenching, Förster-Resonance-Energy-Transfer, etc.) which is shown in figure 2.1 by grey arrows. Alternatively, it can return to the ground state by emitting a fluorescence photon (red arrow). Due to the previous energy loss (caused by internal conversion and vibrational relaxation), the fluorescence photons originating from transitions of electrons from the lowest vibrational state of the first excited singlet state to the ground state, have less energy and therefore longer wavelengths than the absorbed photons. This red-shift of the fluorescence photons is called Stokes shift. Typically, fluorescence lifetimes are between $10^{-9} \mathrm{~s}$ and $10^{-7} \mathrm{~s}$. In addition to these processes, electrons being in an excited state can undergo a spin conversion which is called intersystem crossing (purple arrow), resulting in a molecule being in an excited triplet state. From the lowest vibrational level of the first excited triplet state, the electron can return to the ground state under spin conversion. This phenomenon is called phosphorescence. Since the transition from the excited triplet state to the ground state is quantum mechanical forbidden, the lifetime of the triplet state is much longer compared to fluorescence lifetimes, reaching from milliseconds to hundreds of seconds [63, 82].

Fluorescence can be disturbed by multiple processes resulting in a reduction of fluorescence intensity. A distinction can be made between processes in which the fluorophores are not damaged such as quenching and Förster resonance energy transfer (FRET) and processes in which the fluorophores are irreversibly destroyed. This phenomenon is called photobleaching. Due to interactions with molecular oxygen or other surrounding molecules, the molecule can be chemically modified whilst in the excited state and therefore loses its ability to fluoresce. The disposition of a fluorophore to photobleach depends on its molecular structure and its local environment. Therefore, some fluorphores can undergo many numbers of excitation and emission cycles before they are photo-destroyed, others do not show such a photo-stability [19] (compare [57]). 


\subsubsection{Image formation in confocal scanning fluorescence microscopy}

In confocal fluorescence microscopy, fluorescent-labelled specimens are illuminated by a point source and afterwards the incoherent emitted fluorescence is imaged on a point detector [8, 104]. The advantage of this configuration is that light originating from out of focus regions of the specimen is rejected by the point detector and it therefore allows to distinguish between different focal planes. Thus, it exhibits optical sectioning. However, only one point of the specimen is imaged at a respective time. Hence, the excitation light has to be scanned over the specimen in order to construct a two- or three-dimensional image, by measuring at each scan position the fluorescence intensity reaching the detector [104].

Ideally, the sample point to be illuminated and detected, would be infinitesimally small. However, in classical optical microscopy, the point has a finite size [102 (compare table 2.1). Due to diffraction, its size is limited by the wavelength and the aperture angle of the illumination and collection lens [1]. Usually, the same lens is used for illumination and collection, which is called epi-fluorescence microscopy.

In a good approximation, the imaging of an epi-fluorescence microscope can be considered to be linear and shift invariant. This means that the image of the object is the same as the sum of images from parts of the object (linearity) and the imaging properties remain unchanged over the whole field of view (shift invariance). Mathematically, this can be described via a convolution (see figure 2.2 (b)). For this, the object is considered as consisting of many points, where each point has a size far beyond the diffraction limit. Then, the total image is the sum of the images of each such points and can be calculated according to [11, 85]:

$$
I(x, y, z)=\iiint_{-\infty}^{+\infty} O\left(x-x^{\prime}, y-y^{\prime}, z-z^{\prime}\right) \operatorname{PSF}\left(x^{\prime}, y^{\prime}, z^{\prime}\right) d x^{\prime} d y^{\prime} d z^{\prime}
$$

Here, $I$ represents the final image, $O$ the object and PSF the point spread function. The PSF is the image of a point-like object and therefore describes the transformation of object points through the microscope as illustrated in figure 2.2 (a) [11, 85]. 
point-object

(a)

object

(b)

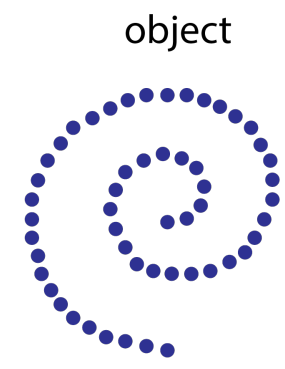

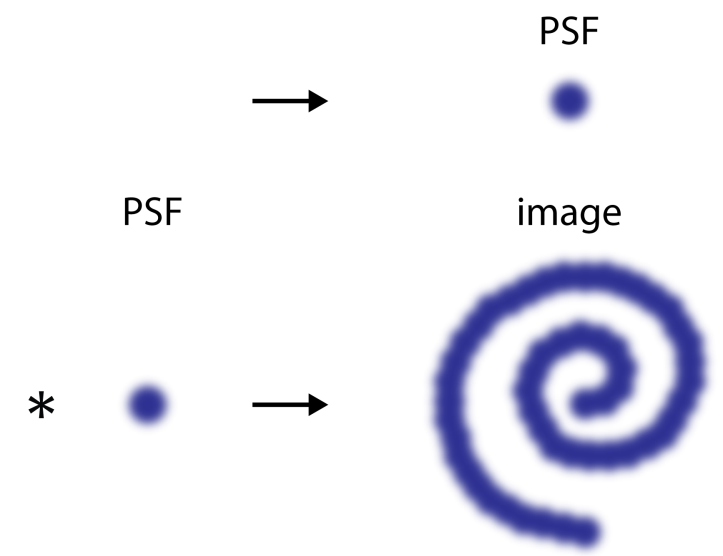

Figure 2.2: Illustration of the imaging of objects through a microscope, adapted from [85] (a) The image of a point-object is called point spread function (PSF) and characterises the transformation of object points through the microscope. Thus, any other object can be described by a convolution of the object with the PSF (b).

Equation (1) can also be expressed via the convolution operator [11]:

$$
I(x, y, z)=O(x, y, z) * \operatorname{PSF}(x, y, z) .
$$

The PSF of a confocal fluorescence microscope can be described by the product of the illumination PSF and the detection PSF [106]:

$$
\operatorname{PSF}_{\text {total }}(x, y, z)=\operatorname{PSF}_{\text {exc }}(x, y, z) \cdot \operatorname{PSF}_{\text {det }}(x, y, z)
$$

The fluorophores have to be excited and afterwards the emitted fluorescence has to be detected in order to create an image of the specimen. Thus, the effective PSF is proportional to the probability of exciting a fluorophore and detecting its photons [37].

Furthermore, since the PSF presents the image of a point-like object, it also gives a direct measure about the resolution of the optical system. Resolution can be defined at the optical system's ability to distinguish objects. If two objects are spaced too closely, they can no longer be identified as individual objects in the image and thus cannot be resolved. The resolution can either be quantified in real space or in Fourier space. In real space, the full width at half maximum (FWHM) of the PSF is usually used to characterise the resolution. Alternatively, the object can be mathematically described by 
a superposition of spatial frequencies with different strengths. During imaging, these spatial frequencies are transferred via the optical system into the image. The efficiency of this transfer is described by the Fourier transform of the PSF, the optical transfer function (OTF). The highest frequency of the object which can be transferred to the image represents the resolution of the optical system in Fourier space [39, 105].

So far only the ideal case of a point-like detection was considered which can be practically realized for example by placing a pinhole in front of a detector. For the ideal case of an infinitely small pinhole and the same excitation and emission wavelength, the resolution in terms of FWHM in lateral and in axial direction can be approximately improved by a factor of $\sqrt{2}$ compared to a wide-field fluorescence microscope with uniform illumination and detection [105]. The resolution of the confocal microscope with open pinhole corresponds to the resolution of a wide-field fluorescence microscope. Therefore, by increasing the size of the pinhole, the resolution of the confocal microscope converges from the resolution of the ideal case to the resolution of a confocal microscope with open pinhole [105].

The effect of the confocal pinhole can be included in the detection PSF in equation (3), to describe the PSF of a confocal microscope with a finite-sized pinhole. For this purpose, the detection PSF ( $\mathrm{PSF}_{\text {det }}$ ) can be expressed by the convolution of the detection $\mathrm{PSF}$ for the case of an infinitely small pinhole $\left(\mathrm{PSF}_{\text {det }}\right)$ with the aperture function of the pinhole [105]:

$$
\operatorname{PSF}_{\operatorname{det}}(x, y, z)=\operatorname{PSF}_{\operatorname{det}}(x, y, z) * D(x, y)
$$

For the case of the same excitation and emission wavelength, the lateral and axial FWHM of a confocal microscope with infinitesimally small as well as with open pinhole can be described by the formulas presented in table 2.1] [103, 105].

\begin{tabular}{l|ll}
\hline \hline FWHM & confocal (open pinhole) & confocal (closed pinhole) \\
\hline lateral & $0.51 \frac{\lambda}{\mathrm{NA}}$ & $0.37 \frac{\lambda}{\mathrm{NA}}$ \\
axia $]^{1}$ & $0.89 \frac{\lambda}{n-\sqrt{n^{2}-\mathrm{NA}^{2}}}$ & $0.64 \frac{\lambda}{n-\sqrt{n^{2}-\mathrm{NA}^{2}}}$ \\
\hline
\end{tabular}

Table 2.1: Overview over the FWHMs of a confocal microscope with open and closed pinhole [105.

\footnotetext{
${ }^{1}$ For small aperture angles [103, the axial FWHM for a open pinhole (o.p.) and for a closed pinhole (c.p.) can be additionally approximated by

$$
\mathrm{FWHM}_{\text {axial, o.p. }} \approx 1.77 \frac{n \lambda}{\mathrm{NA}^{2}} ; \quad \mathrm{FWHM}_{\text {axial, c.p. }} \approx 1.28 \frac{n \lambda}{\mathrm{NA}^{2}} .
$$




\subsection{STED microscopy}

Within this thesis, STED microscopy was employed to enhance the resolution of a confocal fluorescence scanning microscope (see section 2.1). Therefore, a brief overview of STED microscopy is given below.

STED microscopy can be viewed as a special case of the reversible saturable optical fluorescence transitions (RESOLFT) microscopy family. The basic idea of these techniques is to saturate a reversible molecular transition with a spatially shaped intensity distribution featuring a zero intensity point, line or plane to obtain a sub-diffraction sized image spot [45, 51].

RESOLFT microscopy works with molecules having two distinct states A and B, as for example ground and excited states or conformational and isomeric states [39] (see figure 2.3. Referring state A as bright state and state B as dark state, the microscope image is obtained by collecting the signals from the molecules in state A [41].

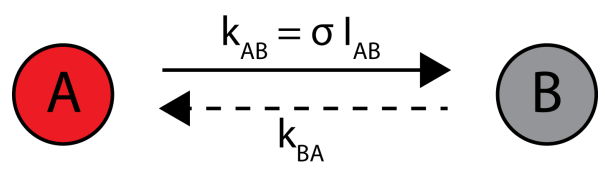

Figure 2.3: RESOLFT microscopy requires molecules exhibiting two distinct state, a bright state A and a dark state B. The transition from sate A to state B has to be optically inducible at a transition rate $\mathrm{k}_{\mathrm{AB}}$, whereas the rate depends on the transition cross section $\sigma$ and the light intensity $\mathrm{I}_{\mathrm{AB}}$. The rate of the transverse transition $\left(\mathrm{k}_{\mathrm{BA}}\right)$ should be negligible, adapted from [46].

Considering a scanning microscope as displayed in section 2.1, the image is formed by scanning the sample with a focused excitation beam. The focused excitation beam transfers samples molecules in state A and the emitted signal by these molecules can be measured. In conventional microscopy, focusing leads to a spot whose size is limited by diffraction. This excitation spot excites all molecules that are located within the spatial extent of this spot (see figure 2.4 for the special case of STED microscopy). Furthermore, all molecules excited to state A emit a signal almost simultaneously and are therefore indistinguishable [41]. Therefore, the spatial separation of molecules (the resolving capability) within the microscope image depends on the spatial extent of molecules being at the same time in state A [41]. If it is possible to confine the area of molecules in state A, the spatial separability of them would be increased [41]. RESOLFT microscopy 
enhances the distinguishability of sample molecules and therefore the resolution by optically transferring all molecules in state A to the state B except for a sub-diffraction sized area [41].

To optically shift the population of state A into state B, the molecular transition between state $\mathrm{A}$ and state $\mathrm{B}$ has to be optically inducible at a wavelength $\lambda_{\mathrm{AB}}$. The wavelength $\lambda_{\mathrm{AB}}$ is usually chosen in such a way that the molecular cross section for the reversed process (transition of state $\mathrm{B}$ into state $\mathrm{A})$ is as small as possible $\left(\sigma_{\mathrm{BA}} \ll \sigma_{\mathrm{AB}}\right)$. The rate of this transition can be described by $\sigma_{\mathrm{AB}} \mathrm{I}_{\mathrm{AB}}$, where $\sigma_{\mathrm{AB}}$ presents the molecular cross section and $\mathrm{I}_{\mathrm{AB}}$ the focal intensity. Assuming a pulsed irradiation with a rectangular pulse of pulse duration $\tau$ as well as a negligible re-excitation by the beam pulse at $\lambda_{\mathrm{AB}}$, the population of state $A$ can be quantified by equation (6) [21, 54, 102]. Here, $\mathrm{N}_{\mathrm{A}}$ and $\mathrm{N}_{0}$ describe the number of molecules in state $\mathrm{A}$ after and before the beam pulse at $\lambda_{\mathrm{AB}}$.

$$
\mathrm{N}_{\mathrm{A}}=\mathrm{N}_{0} \cdot \exp \left(-\sigma_{\mathrm{AB}} \tau \frac{\mathrm{I}_{\mathrm{AB}}}{\hbar \omega}\right)
$$

If the intensity $\mathrm{I}_{\mathrm{AB}}$ is chosen large enough, the population saturates into state B. Usually, a saturation intensity is defined, describing the intensity at which half of the molecules are in state B:

$$
\mathrm{I}_{\mathrm{AB}, \mathrm{sat}}=\frac{\ln 2 \cdot \hbar \omega}{\sigma_{\mathrm{AB}} \cdot \tau}
$$

To confine the area of molecules in state $\mathrm{A}$, the intensity $\mathrm{I}_{\mathrm{AB}}$ is additionally spatially shaped, so that it features at least a single local zero intensity point [40]. Most commonly, a local intensity of zero is generated at the centre of the focal point, referred to as $\vec{r}=\overrightarrow{0}$ [33, 40]:

$$
\mathrm{I}_{\mathrm{AB}}(\vec{r})=\mathrm{I}_{\mathrm{AB}}^{\max } \cdot \mathrm{f}(\vec{r}) \quad \text {, with } \quad f(\vec{r}=\overrightarrow{0})=0
$$

For $\mathrm{I}_{\mathrm{AB}}^{\max } \gg \mathrm{I}_{\mathrm{AB}}$, sat, only molecules in close proximity to the centre of the focal point can be found in state A which depends on the steepness and the spatial shaping of the intensity distribution $f(\vec{r})$. Therefore, the area of molecules in state A can become theoretically infinitely small.

In STED microscopy, fluorescent molecules are used and states A and B represent the first excited singlet state $\left(\mathrm{S}_{1}\right)$ and the ground sate $\left(\mathrm{S}_{0}\right)$ (see figure 2.1). Via stimulated emission, the fluorescence is inhibited and molecules in the excited state only remain in close proximity to the zero intensity point, line or plane of the spatially shaped STED (depletion) beam (see figure 2.4] [42, 46, 50]. 

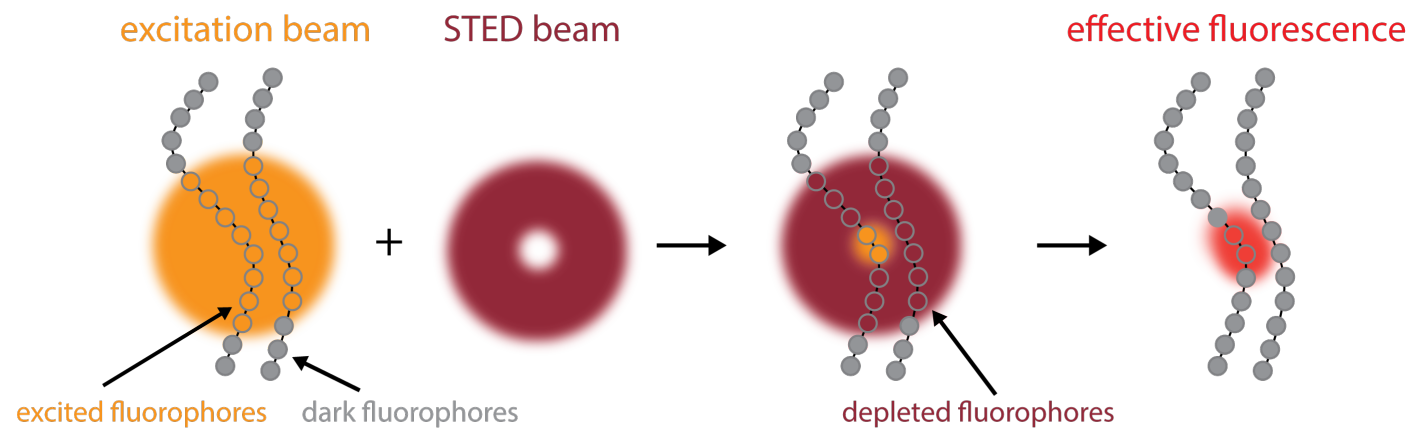

Figure 2.4: Principle of STED microscopy using the example of two filaments marked with fluorophores (grey filled circles), adapted from [43, 67]. An excitation beam excites all fluorophores that are located within its spatial extent (orange filled circles). A spatially shaped depletion beam is then focused on the sample to confine the area of excited molecules. This typically doughnut-shaped STED beam leads to a depletion of excited fluorophores, indicated by circles filled in dark red. In this way, only fluorophores located in close proximity to the centre of the doughnut-shaped beam remain excited and can subsequently fluoresce.

To obtain a spatially shaped STED beam leading to an isotropic (lateral) resolution enhancement in two dimensions, usually, a helical phase mask is applied [24, 51]. The respective phase distribution $\tilde{p}$ of the helical phase mask is illustrated in equation (9) and graphically in figure 2.5 (a) in section 2.3 .

$$
\tilde{p}(r, \phi)=\phi
$$

Employing this phase mask to the STED beam leads to a phase shift of $\pi$ between two mirror symmetrical points. Therefore, when focusing the light, the x-components and the y-components of the electric field cancel each other out on the optical axis. Contributions from z-components of the electric field can be avoided by using circularly polarised light matching the rotation direction of the phase mask. Hence, the combination of a helical phase mask in conjunction with the respective circular polarised light leads to destructive interference on the optical axis. Overall, this results in a ring-shaped- or doughnut-shaped intensity distribution with zero intensity in the centre [24].

To efficiently enhance the resolution in three dimensions, an incoherent combination of several phase masks is needed [50]. The steepness of the intensity distribution along the spatial directions ( $\mathrm{x}, \mathrm{y}$, and $\mathrm{z}$ ) behaves complementary. Therefore, it is not possible to find a phase mask leading to a spatially uniform, narrow intensity distribution. A more detailed discussion on the steepness is provided in the appendix in section $\mathrm{A}$. Therefore, 
the helical phase mask is typically combined with an axial phase mask [34, 150, 78, 83]. The axial phase mask leads to an optimum resolution enhancement in z-direction and is presented in equation (10) [50, 51].

$$
\tilde{p}(r, \phi)= \begin{cases}\pi & \text { for } r \leq r_{0} \\ 0 & \text { elsewhere }\end{cases}
$$

In equation (10), $r_{0}$ describes the radius of an inner circle which is slightly smaller than $\frac{\sqrt{2}}{2}$ for an objective aperture with radius one. The minimum volume in three-dimensions can be obtained by distributing $30 \%$ of the STED light into the axial pattern and $70 \%$ into the helical phase mask [50].

To quantify the resolution of a STED microscope, its PSF can be regarded. The PSF of a STED microscope $\left(\mathrm{PSF}_{\text {eff }}\right)$ can be described as a multiplication of an effective excitation PSF ( $\mathrm{PSF}_{\text {eff,exc }}$ ) with the detection PSF of the confocal microscope (see section 2.1] [33, 36]:

$$
\operatorname{PSF}_{\text {eff }}(x, y, z)=\underbrace{\operatorname{PSF}_{\text {exc }}(x, y, z) \cdot \eta(x, y, z)}_{\operatorname{PSF}_{\text {eff exc }}} \cdot \operatorname{PSF}_{\text {det }}(x, y, z) .
$$

In equation (11), $\mathrm{PSF}_{\text {exc }}$ represents the excitation PSF of the underlying confocal microscope and $\eta$ the suppression factor. The suppression factor reflects the fraction of fluorescence remaining after having employed the STED beam to the sample which can be defined by means of equation (6) and equation (8). Thus, for temporally separated excitation and STED light, a uniform fluorophores' distribution as well as in proximity to the focal point, the suppression factor can be described by [33]:

$$
\eta(\vec{r})=\frac{\mathrm{N}_{\mathrm{f} \mid \mathrm{STED}}(\vec{r})}{\mathrm{N}_{0}}=\exp (-\ln 2 \zeta f(\vec{r})) .
$$

Here, a saturation factor $\zeta$ is defined as the ratio of the maximum STED intensity to the saturation STED intensity [60]:

$$
\zeta=\frac{\mathrm{I}_{\mathrm{STED}}^{\max }}{\mathrm{I}_{\mathrm{STED}}^{\mathrm{sat}}}
$$

For typically applied phase masks, the spatial shaping of the STED beam intensity distribution close to the focal point can be approximated by a parabola [33, 60]. Thus, 
the function $f(\vec{r})$ in equation (8) can be expressed by

$$
f(\vec{r})=4 a r^{2} .
$$

In equation (14), a denotes the pattern steepness which depends on the applied phase mask [33]. In the following, $a_{2 \mathrm{D}}$ is employed for the doughnut-shaped intensity distribution, $a_{\mathrm{z}}$ for the axial depletion pattern and $a_{1 \mathrm{D}}$ for a one-dimensional depletion pattern. By means of this parabolic approximation as well as assuming Gaussian functions for the excitation and detection PSF in equation (11) an effective PSF is obtained which is as well gaussian-shaped [33, 60]. The FWHM of this effective PSF (FWHM $\left.{ }_{\text {STED }}\right)$ depends on the saturation factor $\zeta$ and therefore on the STED laser intensity used as well as on the pattern steepness $a$, the spatial shaping of the STED intensity distribution along a certain direction [33, 60]:

$$
\mathrm{FWHM}_{\text {STED }}=\frac{\mathrm{FWHM}_{\mathrm{conf}}}{\sqrt{1+\mathrm{FWHM}_{\mathrm{conf}}^{2} a \zeta}} .
$$

For high enough STED laser intensities, the width of the effective PSF converges to the expression [33]:

$$
\mathrm{FWHM}_{\mathrm{STED}}=\frac{1}{\sqrt{a \zeta}} .
$$

As illustrated in equation (16), the width of the effective PSF becomes independent of the confocal PSF's width. It only depends on the STED laser intensity as well as on the pattern steepness. Therefore, the width in STED microscopy can theoretically become unlimited small corresponding to an unlimited good resolution.

\footnotetext{
${ }^{2}$ As illustrated in section 2.1 the detection PSF can be described by the detection PSF for an infinitesimally small pinhole convolved with the confocal pinhole.
} 


\subsection{Two-dimensional tomographic STED microscopy}

The aims of this thesis were to improve the performance of two-dimensional tomographic STED (2D tomoSTED) by combining it with image scanning microscopy and to develop it into a three-dimensional imaging technique. Therefore, the concept of $2 \mathrm{D}$ tomoSTED is described below.

2D tomoSTED has been demonstrated in [59, 60] as sample-gentle imaging technique enabling imaging with a light dose reduced by a factor of four compared to the conventional two-dimensional (2D) STED microscopy while maintaining the same image quality. Same image quality means the same resolution in all spatial directions as well as an equal signal strength of the high frequencies.

TomoSTED is based on the idea to use a one dimensional depletion pattern (1D depletion pattern) instead of the doughnut-shaped depletion pattern (2D depletion pattern) used in conventional 2D STED to confine the fluorescent area within the focal plane [60]. The employment of the 1D depletion pattern leads to an effective PSF whose resolution is enhanced only along one direction [60]. 1D depletion patterns can be generated by spatially shaping the phase of the STED beam with a $0-\pi$ phase step [50, 51, 54, 59, 60, 97]. For a resolution increase in $\mathrm{x}$-direction, the 1D phase mask is displayed in equation (17) and illustrated in figure 2.5 (a) ${ }^{3}$.

$$
\tilde{p}(r, \phi)= \begin{cases}\pi & \text { for } \phi \leq \frac{\pi}{2} \vee \phi \geq \frac{3 \pi}{2} \\ 0 & \text { elsewhere }\end{cases}
$$

In addition to the 1D phase mask, light linearly polarised perpendicular to the direction of the phase step has to be employed [97]. With this, the focused STED beam interferes destructively along a line within the focal region [54, 59, 97] leading to a 1D depletion pattern. The 1D depletion pattern for a resolution enhancement in x-direction is illustrated in figure 2.5 (a).

\footnotetext{
${ }^{3}$ The 1D phase mask for the resolution increase along the $\mathrm{y}$-direction is additionally illustrated in figure 5.5 in section 5.1 .3
} 
(a)

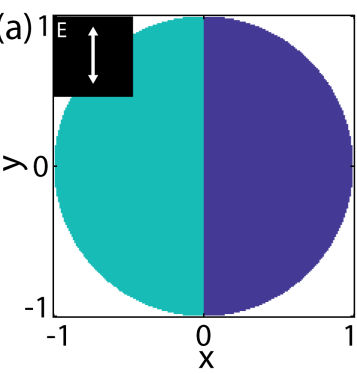

0

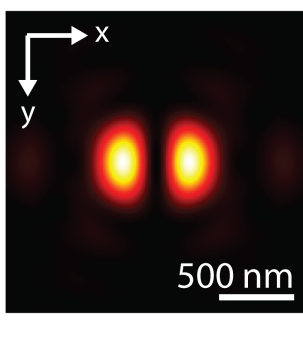

0 (b)

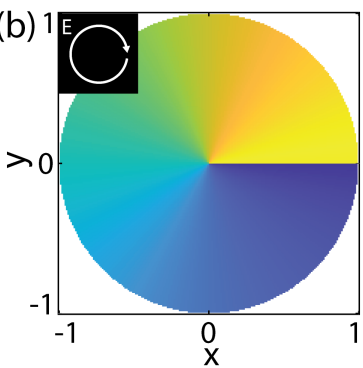

0

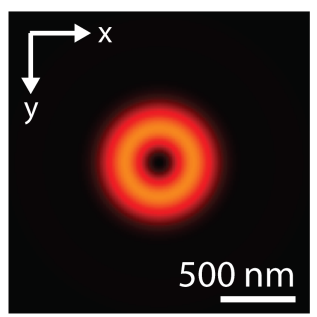

0

Figure 2.5: Comparison of (a) the 1D phase mask and the resulting 1D depletion pattern with (b) the helical phase mask and the corresponding 2D depletion pattern.

Compared to the 2D depletion pattern used in 2D STED (see figure 2.5 (b)), the 1D depletion pattern exhibits higher intensity maximums for the same overall STED intensity. Furthermore, in the high resolution direction of the 1D depletion pattern, the maxima for both methods are located at the same distance from the focal point. A higher maximum intensity located at the same distance indicates a more efficient fluorescence depletion as illustrated in equation (8) in section 2.2. A more efficient fluorescence inhibition corresponds to a higher resolution enhancement. Analogously, the STED laser intensity could be reduced for the 1D case to achieve the same resolution as in 2D STED.

To investigate whether the same resolution as 2D STED can be achieved by using the 1D depletion pattern with a lower STED laser intensity, the effective PSF is considered. As described in section 2.2, the width along the high resolution axis depends on the applied laser intensity as well as on the steepness of the spatial shaping along this direction (see section 2.2] [33]. For a sufficiently high laser power and the approximation of the depletion pattern by a parabola in the considered direction, the FWHM of the effective PSF scales inverse with the depletion pattern steepness $a$ as well as inverse with the employed STED laser power (see equation (16) [33]. Thus, by means of equation (16), a ratio $r$ of the effective PSFs FWHM for an individual tomoSTED PSF to the width of the 2D STED can be defined as

$$
r=\frac{\mathrm{FWHM}_{\mathrm{STED}, 1 \mathrm{D}}}{\mathrm{FWHM}_{\mathrm{STED}, 2 \mathrm{D}}}=\frac{\sqrt{a_{2 \mathrm{D}} \zeta_{2 \mathrm{D}}}}{\sqrt{a_{1 \mathrm{D}} \zeta_{1 \mathrm{D}}}} .
$$

As shown in equation (18), the square of $r$ equals to the ratio of the depletion pattern steepnesses of both variants at identical STED laser power. Therefore, the same FWHM is obtained when the laser power of tomoSTED is divided by the square of $r$ determined 
at an equal laser power (see equation (19)). Thus, $r^{2}$ represents the factor by which the laser power of tomoSTED can be reduced compared to the conventional 2D STED:

$$
\zeta_{1 \mathrm{D}}=\frac{1}{r^{2}\left(\zeta_{1 \mathrm{D}}=\zeta_{2 \mathrm{D}}\right)} \zeta_{2 \mathrm{D}}
$$

A factor $r^{2}$ larger than one would therefore enable to measure with a lower laser power and still achieve the same resolution. To determine the factor $r^{2}$, effective PSFs corresponding to the $1 \mathrm{D}$ and $2 \mathrm{D}$ case were simulated with a saturation factor of 120.4 The ratio of the FWHMs, determined by Gaussian fits to line profiles drawn through the high-resolution axis, yielded a factor $r^{2}$ of about 1.85. To achieve the same resolution improvement in an individual tomoSTED PSF as in 2D-STED along the high-resolution axis, the laser power can therefore be reduced by a factor of about 1.855 .

The individual tomoSTED PSFs are mainly reduced along a single spatial direction. Thus, it is to be expected that the signal of the individual tomoSTED PSFs is higher than that of the conventional 2D-STED when comparing the same resolution enhancement $\left(\mathrm{FWHM}_{\mathrm{STED}, 1 \mathrm{D}}=\mathrm{FWHM}_{\mathrm{STED}}, 2 \mathrm{D}=\mathrm{FWHM}_{\mathrm{STED}}\right)$. Therefore, not only the laser power but also the exposure time could be lowered to achieve the same signal strength in the individual tomoSTED PSFs as in the 2D-STED PSF. Hence, the signal of the individual tomoSTED PSFs is compared with that of 2D-STED in the following.

The PSFs for both methods can be roughly approximated by Gaussian functions [33, 60].

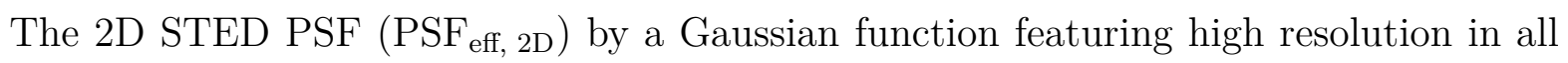
directions [33]:

$$
\mathrm{PSF}_{\text {eff, } 2 \mathrm{D}}=A e^{-4 \ln 2\left(\frac{x^{2}+y^{2}}{\mathrm{FWHM}_{\mathrm{STED}}^{2}}\right)} .
$$

Here, $A$ denotes the amplitude of the Gaussian function which corresponds to the amplitude of the underlying confocal PSF (see equation (11) in section 2.2). The individual tomoSTED PSF exhibits a high-resolution (STED) direction and a diffraction-limited

\footnotetext{
${ }^{4}$ The effective PSFs were simulated using the 'Optical Microscopy Matlab Toolbox' of the Department for NanoBioPhotonics, of the Max Planck Institute for biophysical Chemistry in Göttingen. The 'Optical Microscopy Matlab Toolbox' calculates the effective PSF based on vectorial diffraction theory as presented in section 5.1. For the simulations, an excitation wavelength of $640 \mathrm{~nm}$, an emission wavelength of $680 \mathrm{~nm}$ and a STED wavelength of $775 \mathrm{~nm}$ were used.

${ }^{5}$ Another possibility to determine the scaling factor of the laser power is to compare the different steepness of the two depletion patterns as shown in [60]. The steepness of the pattern can be determined by calculating the second derivative and evaluating its value at the focal point [60]. Both methods lead to the same result in the framework of simulations.
} 
(confocal) direction [59, 60] and can be described by

$$
\mathrm{PSF}_{\text {eff, } 1 \mathrm{D}}=A e^{-4 \ln 2\left(\frac{x^{2}}{\mathrm{FWHM}_{\mathrm{STED}}^{2}}+\frac{y^{2}}{\mathrm{FWHM}_{\text {conf }}^{2}}\right)}
$$

The signal of a two dimensional Gaussian function can be evaluated by integration:

$$
\int_{-\infty}^{\infty} \int_{-\infty}^{\infty} e^{-\frac{1}{2}\left(\frac{x^{2}}{\sigma_{x}^{2}}+\frac{y^{2}}{\sigma_{y}^{2}}\right)} d x d y=2 \pi \sigma_{x} \sigma_{y}=\frac{\pi}{4 \ln 2} \cdot \mathrm{FWHM}_{x} \mathrm{FWHM}_{y}
$$

As illustrated in equation (22), the integral is proportional to the FWHMs of the considered Gaussian functions. Therefore, the signal of the 2D effective PSF is approximately proportional to $\mathrm{FWHM}_{\mathrm{STED}}^{2}$, whereas the signal of an individual tomoSTED PSF is approximately proportional to $\mathrm{FWHM}_{\mathrm{STED}} \cdot \mathrm{FWHM}_{\text {conf }}$. Thus, each individual tomoSTED PSF exhibits a signal which is higher by the factor of $\mathrm{FWHM}_{\text {conf }} / \mathrm{FWHM}_{\text {STED }}$ compared to the 2D effective PSF. This factor corresponds to the factor of resolution enhancement due to STED. Thus, in order to obtain at least the same signal strength in all spatial directions as with the conventional 2D-STED and thus the same image quality, the exposure time of the individual tomoSTED PSFs can be reduced by the signal ratio as compared to 2D-STED ${ }^{6}$ Hence, the exposure time for each individual tomoSTED image can be reduced by the factor of the resolution enhancement due to STED. Both aspects of the individual tomoSTED PSFs, the reduction of STED laser intensity and the reduction of exposure time, have the potential to result in a lower light dose for tomoSTED microscopy [60].

However, so far only one individual tomoSTED PSF was considered. To obtain the same spatial high resolution information as the 2D STED, the high resolution direction of the 1D PSF has to be rotated. The rotation of the high resolution direction is achieved by rotating the depletion pattern. The individual tomoSTED images obtained in this way are then reconstructed to a final highly-resolved image in two dimensions. This principle of tomoSTED is illustrated in figure 2.6.

\footnotetext{
${ }^{6}$ The potential reduction of the exposure time of the individual tomoSTED PSFs presented here differs from the reduction factor used for tomoSTED in [60]. In [60, the exposure time was reduced by the number of pattern orientations used for the tomoSTED measurement. However, together with the optimal number of orientations presented in [60] of $\pi k_{1 D} / 2\left(k_{1 D}\right.$ denoting the resolution increase due to STED), this does not result in at least the same signal strength as in 2D-STED. If the exposure time is reduced by the optimal number of pattern orientations, the signal in the individual tomoSTED PSFs is by $\pi / 2$ lower than that of 2D-STED. At least the same signal strength is only obtained when measuring with a number of pattern orientations equal to the signal ratio, thus the factor of the resolution increase due to STED.
} 

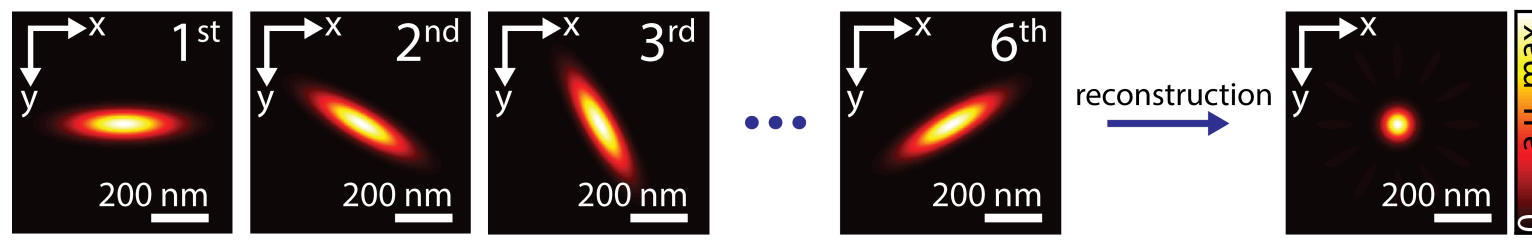

Figure 2.6: Illustration of the principle of tomoSTED. TomoSTED employs 1D depletion patterns leading to individual tomoSTED PSFs exhibiting a high resolution axis in one direction. To obtain high resolution in all spatial direction the high-resolution direction in terms of the 1D depletion pattern is rotated. A final highly-resolved image in two dimensions is afterwards reconstructed from the individual images.

Considering the light dose as the total amount of irradiation applied onto the sample by the STED beam, it can be characterised by the laser power employed to the sample and the total exposure time. If each individual tomoSTED image is recorded with a single exposure time reduced by the signal ratio as compared to that of 2D-STED, the light dose of tomoSTED compared to 2D-STED depends drastically on the number of employed pattern orientations. A much higher number of pattern orientations as the signal ratio will not results in a higher light dose compared to 2D-STED. However, the number of pattern orientations has to be chosen high enough in order to achieve the same resolution in all spatial directions as in 2D-STED and thus the same image quality. 


\section{Material and Methods}

\subsection{Experimental realization}

To be able to experimentally realize the objectives of this thesis, a suitable microscope setup was built. The setup had to meet the requirements that image scanning microscopy measurements, tomoSTED measurements as well as the combination of both are possible. Additionally, to be able to compare tomoSTED measurements with the conventional STED variants in two- and three dimensions, conventional STED measurements should also be realizable with the setup. Furthermore, the setup was designed in such a way that the oil objective lens (100x, NA $=1.4$, UPLSAPO 100XO, Olympus, Japan), used in this thesis, could be replaced by a water objective lens $(60 \mathrm{x}, \mathrm{NA}=1.2)$. The experimental setup is shown schematically in figure 3.1 and is in the following explained in more detail.

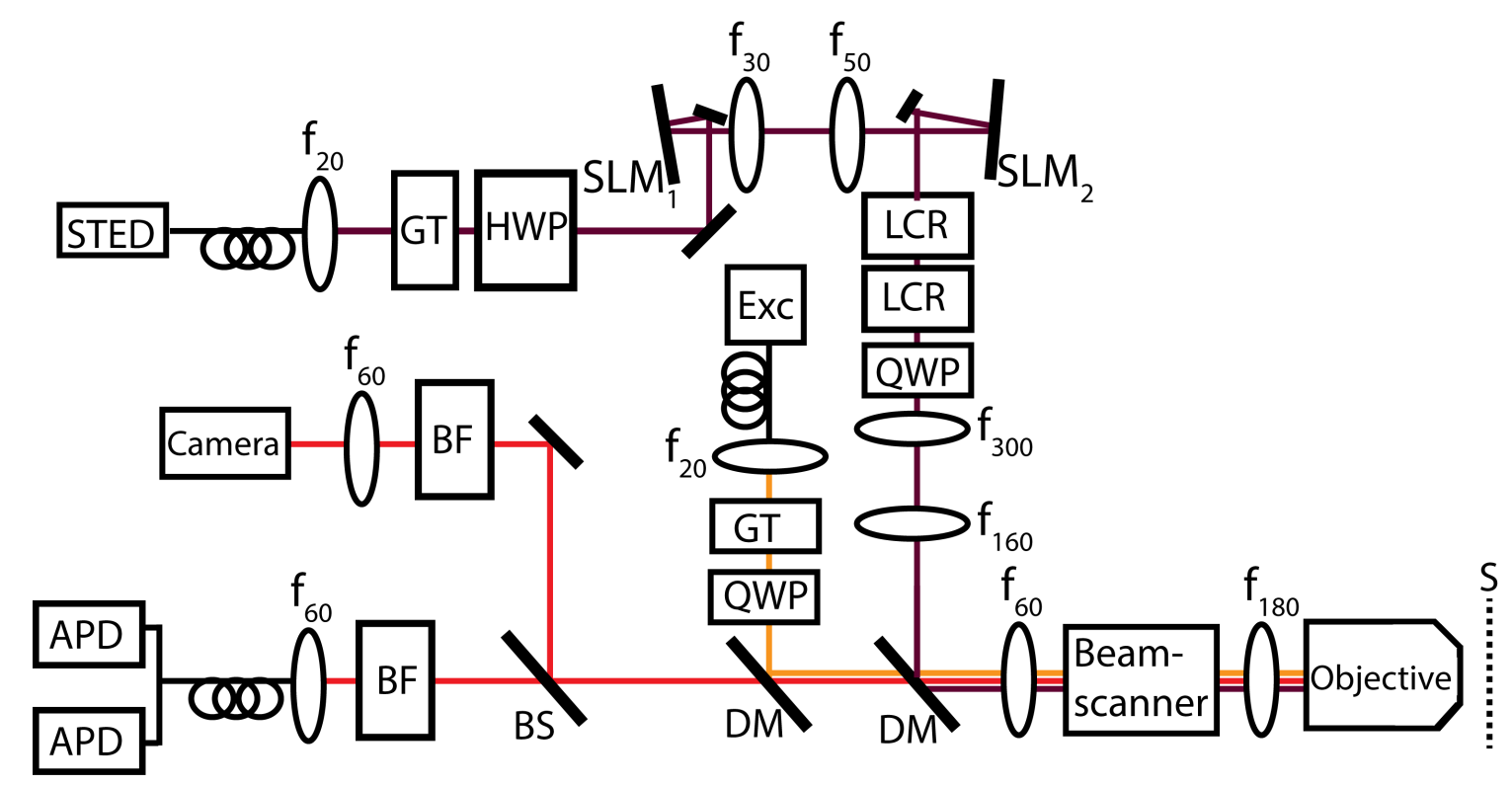

Figure 3.1: Schematic illustration of the used experimental setup (STED: STED laser unit, Exc: excitation laser unit, SLM: spatial light modulator, LCR: liquid crystal retarder, APD: avalanche photodiode, GT: Glan-Thompson-prism, HWP: half waveplate, QWP: quarter waveplate, DM: dichroic mirror, BS: pellicle beam splitter, BF: emission bandpass filter f: focal length)

The microscope setup can be divided into three main path ways of wavelengths of different colours, an excitation light path to excite a fluorescent sample (orange), a STED depletion path (dark red) to suppress the fluorescence within a selected region and a detection path (red) to detect the remaining fluorescence. Via two dicroic mirrors (F43- 
643 and F73-750T, AHF analysentechnik AG, Germany), the excitation beam and the STED beam are overlapped and focused via the objective lens into the sample plane. An additional beam scanner (Abberior Instruments GmbH, Germany) installed in front of the objective lens was used to scan both beams over the sample. After the excitation and depletion process, the fluorescent light was separated from the excitation and STED beam by the two dicroic mirrors and then entered the detection path.

To get laser light into the excitation path as well as into the depletion path, a $640 \mathrm{~nm}$ excitation laser diode (LDH-P-C-640B, PicoQuant, Germany) with a pulse length of $100 \mathrm{ps}$ at a repetition rate of $80 \mathrm{MHz}$ and a STED laser (Katana $08 \mathrm{HP}$, Onefive GmbH, Switzerland) with a wavelength of $775 \mathrm{~nm}$, a pulse length of $600 \mathrm{ps}$, and a repetition rate of $80 \mathrm{MHz}$ are coupled via polarization maintaining single-mode fibers (PMC-630-4.1NA012-3-APC-560-P and PMC-E-780-5.1-NA012-3-APC.EC-500-P, Schäfter + Kirchhoff $\mathrm{GmbH}$, Germany) into the setup. To be able to modulate the intensity of the lasers beam, an acousto-optic modulator (AA.MT110-1.5-VIS and AA.MT110-A1.5-IR, AA Opto Electronic, France) is installed in front of each fiber coupling. To ensure that the sample is first excited by the excitation beam and after a short time delay depleted by the STED beam, the excitation laser diode is triggered by the STED laser and the time delay between excitation and STED pulse is adjusted electronically via the software Imspector (Abberior Instruments GmbH, Germany).

After being coupled into the main part of the setup, the excitation light is collimated by a collimator (Schäfter + Kirchhoff GmbH, Germany) possessing a focal length of $20 \mathrm{~mm}$. Hereby, the focal length were chosen in such a way that the excitation beam over-illuminates the backfocal plane of both objective lenses. To purify the polarisation after the fiber, a Glan-Thompson prism was installed afterwards. Additionally, a quarter wave plate was placed to make the polarisation circular. Circular polarisation provides the most efficient excitation of fluorophores [59].

In the STED path, not only a collimator lens with a focal length of $20 \mathrm{~mm}$ (Schäfter + Kirchhoff GmbH, Germany) was installed, but additional telescopes. The focal length of the lenses were chosen such that the SLMs lie in conjugate planes to the backfocal plane of the objective lens, that the backfocal plane is over-illuminated for either choice of the objective lens and that the projection of the back focal plane onto the two SLMs exhibits a sufficiently high pixelation. The pixelation affects the shape of the depletion pattern, in particular the depletion pattern steepness as investigated in [59]. Here, the 
smallest pixelation of the backfocal plane's diameter projected onto the SLMs was chosen to be 131 pixels, resulting in an undistorted depletion pattern according to [59].

To modulate the phase of the STED-beam, two spatial light modulators were incorporated into the setup SLM $_{1}$ (X13268-02, Hamamatsu Photonics Deutschland GmbH, Germany) and SLM 2 (XY P256 HS, Boulder Nonlinear Systems, USA). The 1D depletion pattern for tomoSTED (2D- and 3D tomoSTED as well as ISM-tomoSTED) were generated using the $\mathrm{SLM}_{2}$ which allows to switch the single depletion pattern orientations at a rate of $200 \mathrm{~Hz}$. The $\mathrm{SLM}_{1}$ was mainly built into the setup to perform conventional 3D STED microscopy. In conventional 3D STED microscopy, two phase masks are combined to achieve a minimum volume in $3 \mathrm{D}$ (see section 2.2).

To create the depletion pattern, respective voltages were applied to the pixels of the SLMs leading to a spatially phase-shaped STED beam. To use only phase modulated light in the following, the voltage-pixel mask of the STED phase mask was combined with a voltage mask of a diffraction grating. Subsequently, only the STED light was used, which was diffracted to the first order. The generation of depletion patterns for STED microscopy requires not only the spatial modulation of the STED beam, but also a corresponding adjustment of its polarisation (see section 2.2). The polarisation was adapted using a combination of two liquid crystal retarders (LRC-200-IR1-TSC, Meadowlark Optics, USA) with a quarter-wave plate. This combination enables the generation of any linear polarisation state required for tomoSTED as well as circularly polarised light necessary for the conventional STED variants. During a tomoSTED as well as an ISM-tomoSTED measurement, the 1D phase mask and the respective linear polarisation was rotated after each line scan. The rotation of the phase mask as well as of the polarisation after each line scan was controlled via Python. In this way, the same image with different oriented depletion patterns could be obtained.

To detect the fluorescence, two options were built into the microscope, a camera (Zyla 4.2 Plus, Andor Technology, Belfast) and two Avalanched Photo Diodes (APDs, SPCMAQRD-13-FC, Excelitas Technologies Corp., USA). For this, the detection path was split via a pellicle and about $60 \%$ of the fluorescence emission was led to the two APDs and about $40 \%$ of the detection light reached the camera. In order to obtain the greatest possible comparability between tomoSTED and ISM-tomoSTED, the measurements for the comparison of the two methods were carried out with the camera only, although no camera is necessary for tomoSTED. Furthermore, an additional emission band pass filter 
(F49-691, AHF analysentechnik AG, Germany) was installed in front of each detection option to prevent remaining excitation and STED light from reaching the detectors.

During an image scanning microscopy measurement, the fluorescence emitted by the sample at each scan position has to be collected by the camera. To guarantee that the camera takes an image at every scan position, the camera was electronically triggered by the pixel trigger provided by the software Imspector (Abberior Instruments GmbH, Germany). In addition, the acousto-optic modulators were triggered by the camera. In this way, the sample was only illuminated and therefore only emitted light when the camera was ready to detect the fluorescence.

\subsection{Polarisation adjustment using liquid crystal retarders}

To create the 1D depletion pattern, not only the phase of the STED beam has to be spatially shaped, but the polarisation has to be adjusted as well. To obtain zero intensity within the focal point, the polarisation has to be linear aligned perpendicular to the $1 \mathrm{D}$ phase step. Therefore, in order to realize all possible 1D depletion patterns, all linear polarisation states have to be generatable. Previous experimental realizations of tomoSTED [59, 97] used a pair of Pockels cells to adjust the polarisation of the 1D depletion pattern. Liquid crystal retarder (LCR) have the advantage that they act even under low external fields applied ([52], p.100). Thus, in contrast to Pockels cells which need voltages in the $\mathrm{kV}$ range [97], a few volts are sufficient for LCRs to re-orient and thus enable a change in the polarisation of light. Therefore, LCRs (see section 3.2.1) are probed for tomoSTED within this thesis and are examined concerning their capability to realize all linear polarisation states (see section 3.2.3). Moreover, the polarisation adjustment should be as fast as possible. Thus, the reorientation time of the LCRs is investigated (see section 3.2.4). In the ideal case, they should possess a comparable speed as the SLM which is $200 \mathrm{~Hz}$ ).

\subsubsection{Liquid crystal retarders}

LCRs consists of a liquid crystals which are birefringent ([52], p.3). Thus, dependent on the orientation of the liquid crystals' optical axis an incoming light beam can experience a phase modulation without changing the polarization state, a phase retardation or a rotation of the polarisation ([52], p.3). Moreover, their orientation can be re-orientated 
by means of an external field, such as an electric field, a magnetic field or an optical field [52], p.3). Thus, the polarisation of incoming light can be changed by applying a different external field to the liquid crystals. When the external field is removed, the liquid crystals relax back to their initial orientation[52], p.100).

The LCRs employed within this thesis consists of a parallel aligned nematic liquid crystal cell. This alignment is achieved by confining the cell between two glass substrates coated by a polymide layer [4]. The glass substrates are additionally coated by indium-tin-oxide acting as electrodes and thus allow to apply an electric field onto the liquid crystal cell.

\subsubsection{Description of the polarisation states}

The polarisation of light describes the temporal course of the electric field's direction ([80], p.194). Electromagnetic waves can be considered as transversal waves whose electric an magnetic field vectors lie normal to the direction of propagation ([7], p.24). Thus, assuming an electromagnetic wave propagating in z-direction, the electric field vector of the wave can be expressed by a component in x-direction and one in y-direction ([80], p.195):

$$
\vec{E}(z, t)=E_{x}(z, t) \overrightarrow{e_{x}}+E_{y}(z, t) \overrightarrow{e_{y}}
$$

For a monochromatic plane wave with an angular frequency $\omega$ and a wave vector $\mathrm{k}$, the $\mathrm{x}$ - and y-component of the electric field vector can be described by ([80], p.195)

$$
E_{x}(z, t)=E_{0, x} \cos \left(\omega t-k z+\delta_{x}\right)
$$

and

$$
E_{y}(z, t)=E_{0, y} \cos \left(\omega t-k z+\delta_{y}\right) .
$$

As illustrated by equations (23) to (25), the direction of the electric field vector and thus the polarisation of the electromagnetic wave depends on its amplitudes in $\mathrm{x}$ - and y-direction $\left(E_{0, x}, E_{0, y}\right)$ as well as on the phase difference between these two components $\delta=\delta_{y}-\delta_{x}$. Re-writing equations (24) and (25) leads to the position and time independent parametric equation of the ellipse (([80], p.195-196),([7], p.25)):

$$
\left(\frac{E_{x}}{E_{0, x}}\right)^{2}+\left(\frac{E_{y}}{E_{0, y}}\right)^{2}-2 \frac{E_{x} E_{y}}{E_{0, x} E_{0, y}} \cos (\delta)=\sin ^{2}(\delta) .
$$


Hence, at a fixed position $\mathrm{z}$, the electric field vector rotates periodically in the $\mathrm{x}-\mathrm{y}$ plane following an ellipse (([80], p.196). Therefore, the polarisation of the electromagnetic wave can be characterised by the orientation and the shape of this ellipse (see figure 3.2 (a)). The orientation of the ellipse can be quantified by the direction of the ellipse's major axis. The major axis's direction can be described by the angle $\phi$ lying between the major axis and the $\mathrm{x}$-axis ([7],p.26). The angle $\phi$ is related to the amplitudes of the electric field components and to their phase difference according to ([7],p.28)

$$
\tan (2 \phi)=\frac{2 E_{0, x} E_{0, y} \cos (\delta)}{E_{0, x}^{2}-E_{0, y}^{2}} .
$$

The shape of the ellipse is determined by the ratio of the minor axis to the major axis which can be quantified by the ellipticity angle $\chi(([80], p$ 196),([7], p. 32)):

$$
\sin (2 \chi)=\frac{2 E_{0, x} E_{0, y} \sin (\delta)}{E_{0, x}^{2}+E_{0, y}^{2}} .
$$

With these two angles, each polarisation state of a plane monochromatic wave can be characterised.

(a)

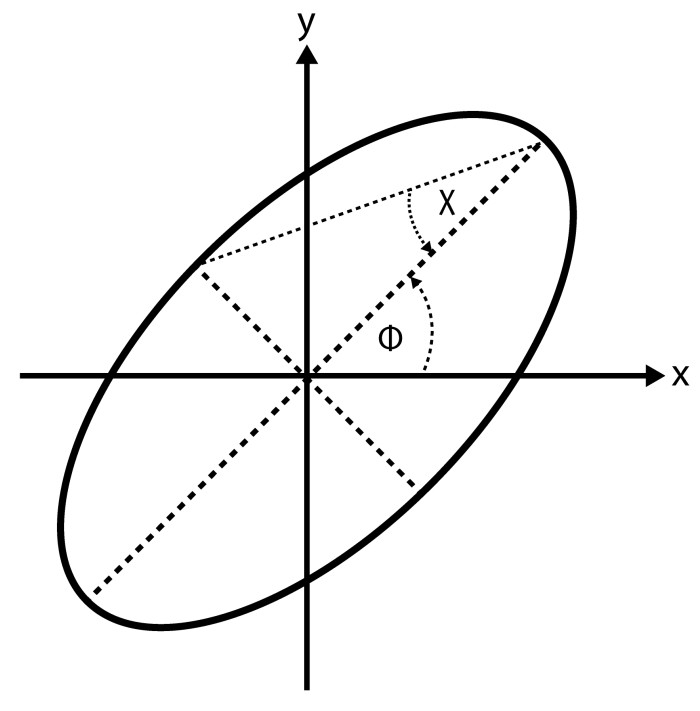

(b)

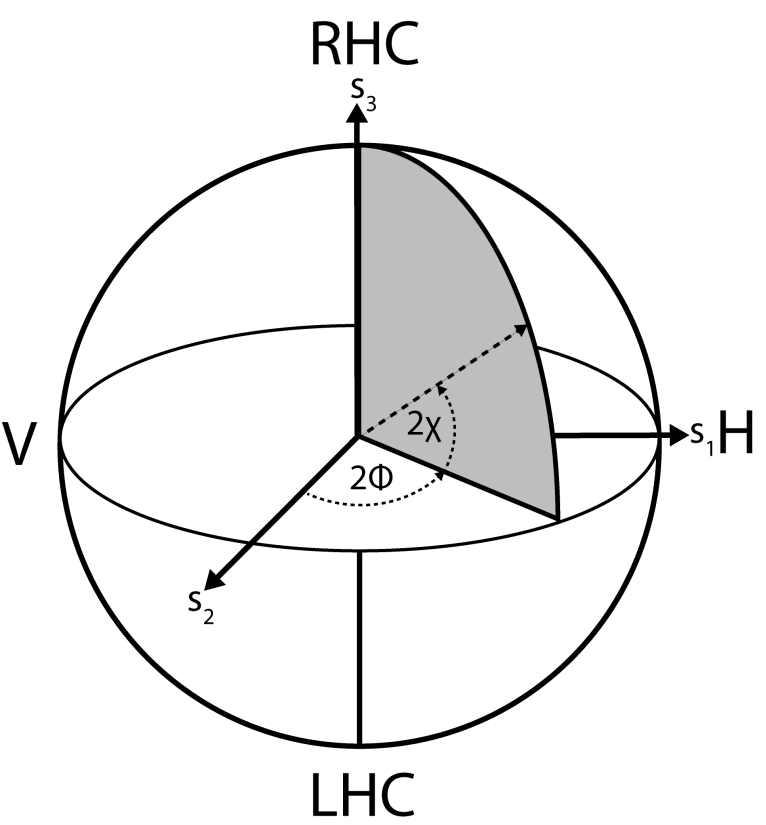

Figure 3.2: Illustration of the polarisation state by means of (a) the polarisation ellipse adapted from [55] and (b) by the Poincaré sphere adapted from ([55],([7], p.32))

Another representation of the polarisation state is the so-called Poincaré's sphere ([7], p.31) as illustrated in figure 3.2 (b). The Poincaré's sphere uses the characterisation of the 
polarisation sate of a monochromatic wave induced by G.G.Stokes which is given by the Stokes parameters ([7], p.31). The Stokes parameters are displayed in equation 29 together with their relations to the angle $\phi$ and the ellipticity angle $\chi$ ([7], p.31-32).

$$
\begin{aligned}
& s_{0}=\sqrt{s_{1}^{2}+s_{2}^{2}+s_{3}^{2}}=E_{0, x}^{2}+E_{0, y}^{2} \\
& s_{1}=s_{0} \cos (2 \chi) \cos (2 \phi)=E_{0, x}^{2}-E_{0, y}^{2} \\
& s_{2}=s_{0} \cos (2 \chi) \sin (2 \phi)=2 E_{0, x} E_{0, y} \cos (\delta) \\
& s_{3}=s_{0} \sin (2 \chi)=2 E_{0, x} E_{0, y} \sin (\delta)
\end{aligned}
$$

Here, the angle $\phi$ lies within the range of $0 \leq \phi \leq \pi$ and $\chi$ in the range of $-\frac{\pi}{4} \leq \chi \leq \frac{\pi}{4}$. The representation of the Stokes parameters $s_{1}$ to $s_{3}$ using the angles $\phi$ and $\chi$ resemble the assignment of spherical coordinates to Cartesian coordinates. Thus, each polarisation state, characterised by the coordinates $s_{1}, s_{2}, s_{3}$, can be represented by a point on a sphere with radius $s_{0}$. Here, $s_{0}$ is proportional to the intensity of the wave ([7], p.32). Linear polarisation exhibits a phase difference of $\delta=0, n \pi$ for $n \in \mathbb{Z}$. Therefore, the third Stokes parameter $s_{3}$ becomes zero. Thus, all linear polarisation states lie on the equator of the Poincaré sphere. Furthermore, the first two Stokes parameters $\left(s_{1}, s_{2}\right)$ vanish for circular polarised light $\left(\delta= \pm \frac{\pi}{2}\right)$. Hence, right handed and left handed circular polarised light can be found on the north pole and on the south pole of the sphere, respectively. All other elliptical polarisation states lie in between the poles and the equator and are separated in right-handed polarisation states, located above the equator plane, and left-handed polarisation states positioned below the equator plane ([7], p. 32-33).

\subsubsection{Generation of linear polarised light with liquid crystal retarders}

As described in [59], any linear polarisation sate can be adjusted by a combination of two Pockels cells. Thus, the adjustability of every conceivable linear polarisation state with a combination of two LCRs was examined similar to [59] and is described in the following.

To test the generation of linear polarisation states with the combination of two LCRs, a laser diode with a wavelength of $670 \mathrm{~nm}$ was used. The polarisation of this laser diode light was set vertically by means of a Glan Thompson prism. The optical axis of the first LCR was rotated by $45^{\circ}$ compared to the vertically polarised light entering the LCR. The optical axis of the LCRs employed within this thesis lie along the slow axis of the 
liquid crystal molecules [4. Decreasing the voltage applied to the LCR starting with the voltage corresponding to a phase retardance of 0 leads to changes of the polarisation of light after the LCR. If the polarisation is measured and visualised on the Poincaré sphere, a rotation of the polarisation around the y-axis can be observed, as illustrated in figure 3.3 (a) with red dots. If the second LCR's optical axis is aligned parallel to the vertically polarised input light, the polarisation state rotates around the $\mathrm{x}$-axis on the Poincaré sphere, as displayed with blue dots in figure 3.3 (a). Furthermore, if the retardance of the second LCR is set to the that of a quarter wave plate, the polarisation state after the second LCR lies on the equator and thus linear polarisation is reached. The position on the equator depends on the retardance adjusted to the first LCR. By changing the retardance of the first LCR and using the retardance of a quarter wave plate for the second LCR, it is therefore possible to obtain theoretically any linear polarisation states.

(a)

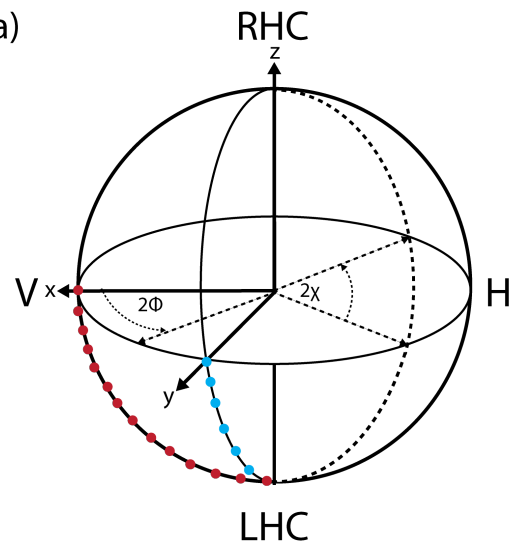

(c)

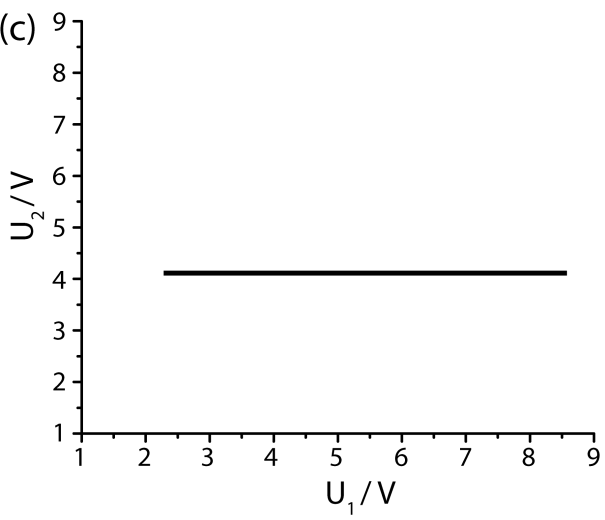

(b)

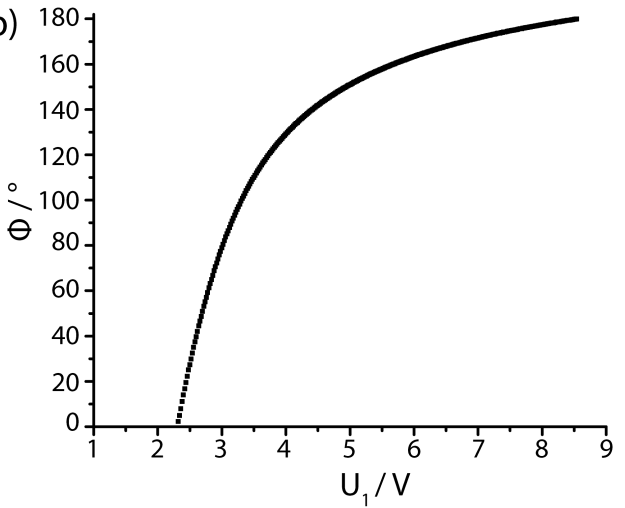

(d)

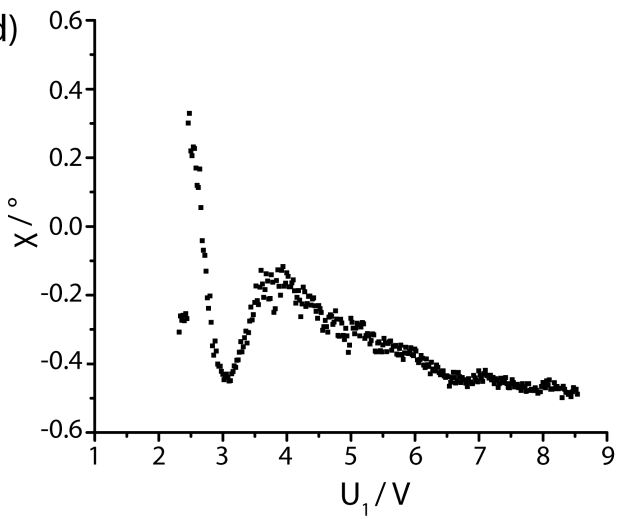

Figure 3.3: Adjusting of linear polarisation using a combination of two LCRs. (a) Working principle of the generation of linear polarised light, (b) measured polarisation orientation via the angle $\phi$ in relation to the voltage applied onto the first LCR, (c) voltage of the second LCR required to reach the linear polarisation equator depending on the adjusted voltage onto the first LCR and (d) the ellipticity angle $\chi$ measured for the generated polarisation states in relation to the voltage applied onto the first LCR. 
To validate the generation of linear polarisation states, the polarisation of the light after the second LCR was measured by means of a polarisation analyser (Schäfter+Kirchhoff GmbH, Germany). This polarisation analyser measures the polarisation according to the Stokes parameters (see section 3.2.2] [55]. Thus, the polarisation of the light measured in relation to the angle $\phi$ and $\chi$. Within the measurement software, the $\mathrm{x}$-axis of the Poincaré sphere is chosen as the axis of vertically polarised light [55] (see figure 3.3 (a)). Thus, here the polarisation orientation angle $\phi$ corresponds to zero for vertical polarised light. The procedure of generating the linear polarisation states using Pockells cells was automated by B. Vinçon by means of a LabVIEW routine [97]. This routine was applied to find for each voltage set on the first LCR the respective linear polarisation state. For each voltage applied to the first LCR, the voltage of the second LCR is varied until linear polarisation of the light is reached. The goodness of the linear polarisation is mapped via the ellipticity angle $\chi$ measured for each voltage applied to the second LCR. According to [59], an ellipticity angle of $0.6^{\circ}$ leads to an undistorted $1 \mathrm{D}$ depletion pattern. Thus, the altering of the second LCR's voltage is stopped when $\chi$ is lower than this threshold value. The in this way found polarisation states in relation to the voltage applied to the first LCR are presented in figure 3.3 (b). As shown in figure 3.3 (b), the total range of linear polarisation ranging from $0^{\circ}$ to $180^{\circ}$ is adjustable with the combination of two LCRs. Moreover, the polarisation sates exhibiting an ellipticity value smaller than $0.6^{\circ}$ (see figure 3.3 (d)) could be generated with a constant voltage value applied to the second LCR (see figure 3.3 (c)) as predicted from theory. Thus, LCRs are suitable for the generation of linear polarisation states for the 1D depletion patterns of tomoSTED.7

\subsubsection{Liquid crystal retarder response time}

The time required by the LCRs to change the phase of light can be measured optically. For this, the LCR can be placed in between a polariser and an analyser ([52], p.104)) and the change of the intensity transmittance in relation to the time can be measured [4]. For a parallel aligned nematic crystal cell which is sandwiched between two crossed polarisers,

\footnotetext{
${ }^{7}$ In [97], another arrangement for the generation of linear polarisation was presented. Two Pockels cells were combined with an additional quarter wave plate leading the same range of adjustable linear polarisations. However, when integrating them into a microscope setup with multiple polarisation sensitive components, the generation of the linear polarisation states could be improved by the placing of the additional quarter wave plate. Therefore, an additional quarter wave plate was placed behind the two LCRs in the final experimental setup (see section 3.1).
} 
the time dependent intensity transmittance can be described by [4],([52], p.164))

$$
I(t)=\sin ^{2}\left(\frac{\delta(t)}{2}\right)
$$

Here, $\delta(t)$ denotes the phase change of the light which is induced by applying an electric field onto the LCR.

When the electric field is switched off by removing the voltage applied to the LCR, the phase change caused by the LCR can be approximated by an exponential decay [99],([52], p.164)):

$$
\delta(t) \approx \delta_{0} \exp \left(-\frac{t}{\tau}\right)
$$

In equation (31), $\delta_{0}$ represents the initial phase retardation and $\tau$ represents the optical phase decay time which can be determined from the measured intensity transmittance.

When an electric field is applied to the LCR, a simply exponential approximation of the phase retardation is only valid in a very short time window [99]. An approximation of the phase retardation's behaviour in the case of an electric field applied onto the LCR was derived by 99

$$
\delta(t) \approx \frac{\delta_{0}}{1+\left(\frac{\phi_{\infty}^{2}}{\phi_{0}^{2}}-1\right) \exp \left(-\frac{t}{\tau}\right)} .
$$

Here, $\phi_{\infty}$ denotes the angle of the LCR's optical axis at the time $t=\infty$ and $\phi_{0}$ the respective angle at $t=0$, thus the initial optical axis orientation. Electrical forces acting on the molecule are stronger than intermolecular restoring forces, thus the re-orientations of the liquid crystals is faster when applying an electric field and is slower when the electric field is removed[4].

The experimental implementation of this optical measurement idea used in this thesis is illustrated in figure 3.4 .

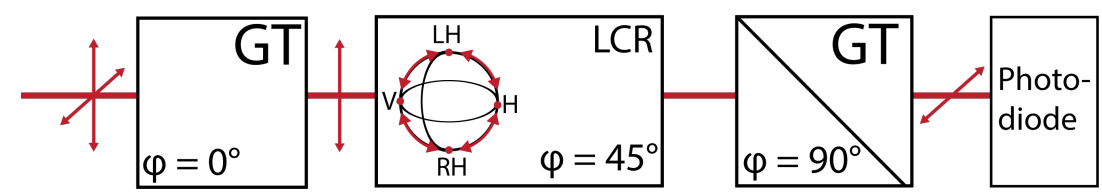

Figure 3.4: Experimental setup for measuring the optical response time of the liquid crystal retarders, (GT: Glan Thompson, LCR: Liquid Crystal Retarder, V: vertically polarised, LH: left-handed circularly polarised, H: horizontally polarised, RH: right-handed circularly polarised) 
The response time of the LCRs were measured with the configuration of the first LCR described in the previous section (see section 3.2.3). The optical axis of the LCR was rotated by $45^{\circ}$ with respect to the vertically polarised incident light, so that left- and right handed circularly polarised light, horizontally polarised light and all other polarisation states that lie between the above could be generated (see figure 3.4). After having passed the LCR, the light reached another Glan Thompson prism whose optical axis was aligned perpendicular to that of the first one. Thus, only horizontally polarised light was let through the second Glan Thompson prism. Therefore, the second Glan Thomspon prism enabled to measure the change in the polarisation of light generated by the LCR. The light intensity passing through the second Glan Thompson prism as measured with a photodiode placed behind the prism. The signal of the photodiode was then read out with an oscilloscope.

To examine the intensity change over time, a voltage step with a duration of $80 \mathrm{~ms}$ was applied to the LCR. As the response time decreases for increasing temperature [4], it was also heated up to the maximum possible temperature of $50^{\circ}$. Several polarisation transitions were observed and the slowest transition was found at a retardance change of the LCR from $\lambda$ to $\lambda / 2^{8}$ which is shown in figure 3.5 .

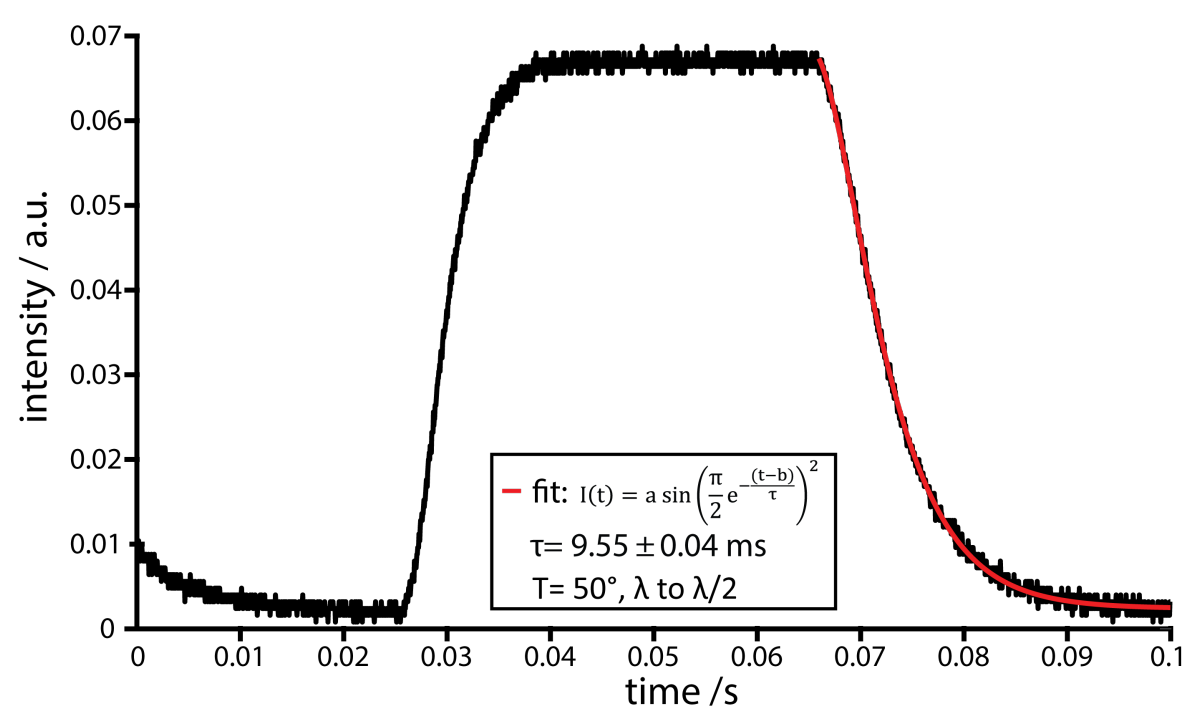

Figure 3.5: Measured change in the intensity transmittance over time as well as at $50^{\circ} \mathrm{C}$ when applying a voltage step with a duration of $80 \mathrm{~ms}$ to the LCR with voltages corresponding to a LCR retardance change from $\lambda$ to $\lambda / 2$.

As shown in figure 3.5, transmittance increases when the retardance of the LCR is

\footnotetext{
${ }^{8}$ In addition, the following transitions were examined as well: a retardance change from zero to $\lambda / 4$,
} from zero to $\lambda / 2$, from $\lambda / 4$ to $\lambda / 2$, from $3 \lambda / 4$ to $\lambda / 2$. 
changed from $\lambda$ to $\lambda / 2$ and decreases at the end of the applied voltage step. It can be observed in figure 3.5 that the transition back to the LCR's initial state exhibits a longer response time than the transition to the retardance of $\lambda / 2$. The slowest transition should posses a comparable speed as the SLM. Therefore, only the time required for the transition back to the initial state is examined further in the following. This transition time was analysed in more detail by fitting equation (30) as well as equation (31) to the measured data. Via the fit presented with the red line in figure 3.5, an optical phase decay time of $(9.55 \pm 0.04) \mathrm{ms}$ was obtained for the slowest observed polarisation adjustment. Thus, the response time is two times slower than the switching rate of the SLM. However, with the help of an additional quarter-wavelength plate, all linear polarisation states can be generated by merely switching the voltage between a retardance of zero and of $\lambda / 2$. A phase change between zero and $\lambda / 2$ is approximately twice as fast as the change between $\lambda$ and $\lambda / 2$. This would make the polarisation adjustment for the $1 \mathrm{D}$ depletion pattern as fast as rotating the phase mask using the SLM. Therefore, LCRs can be used for polarisation adjustment in tomoSTED.

\subsection{Reconstruction of tomoSTED images}

TomoSTED microscopy is based on one-dimensional depletion patterns. In order to get high resolution information in all spatial directions, the depletion pattern has to be rotated. Thus, a final highly-resolved image has to be reconstructed from all images recorded at different orientations. In [59, 60], the maximum-value reconstruction method as well as the Richardson-Lucy deconvolution method have been identified as suitable reconstruction methods for tomoSTED. Thus, they are as well applied within this thesis and described in the following.

\subsubsection{Maximum-value reconstruction}

The maximum-value reconstruction [58, 59, 60, 81] considers the image formation process of a microscope in a Fourier optical way [81]. Regarding the image as composed of spatial frequencies with different amplitudes, the final image can be reconstructed by comparing the Fourier transforms of the individual images and keeping only the largest contribution from all individual orientations at each spatial frequency for the final image [59, 81]:

$$
\mathcal{F}(I)=\max _{i}\left(\mathcal{F}\left(I_{i}\right)\right)
$$


For this, as indicated in equation (33), the images of the individual tomoSTED images $I_{i}$ are Fourier transformed. The final image $I$ is then obtained pointwise in Fourier space [59, 81]. For each spatial frequency, the magnitudes of the individual Fourier transforms are compared and the largest magnitude is transferred to the final imag [81]:

$$
\max _{i}\left(\mathcal{F}\left(I_{i}\right)\right)=\left|\mathcal{F}\left(I_{k}\right)\right| \geq\left|\mathcal{F}\left(I_{i}\right)\right| \quad \forall i \in\{1, . ., n\}, i \neq k
$$

The reconstructed image in real space is afterwards obtained by an inverse Fourier transform.

In addition, the reconstructed image can be described by a convolution of the object with an effective PSF (see equation (35)) [58]. Therefore, the maximum-value reconstruction represents a linear fusion process. This linearity becomes evident when the individual images $I_{i}$ are expressed as the convolution of the object $O$ underlying the images with the individual PSFs:

$$
\begin{aligned}
\mathcal{F}(I) & =\max _{i}\left(\mathcal{F}(O) \cdot \mathcal{F}\left(\mathrm{PSF}_{i}\right)\right) \\
& =\mathcal{F}(O) \cdot \max _{i}\left(\mathcal{F}\left(\mathrm{PSF}_{i}\right)\right) \\
& =\mathcal{F}(O) \cdot \mathcal{F}\left(\mathrm{PSF}_{\text {eff }}\right)
\end{aligned}
$$

As shown in equation (35), the effective PSF results from the maximum amplitude projection of the individual PSFs in Fourier space:

$$
\operatorname{PSF}_{\text {eff }}=\mathcal{F}^{-1}\left(\max _{i}\left(\mathcal{F}\left(\mathrm{PSF}_{i}\right)\right)\right)
$$

\subsubsection{Richardson-Lucy deconvolution}

The Richardson-Lucy deconvolution method, suggested by W.H. Richardson [74] and L.B. Lucy [65], is a nonlinear restoration methods [68] that estimates the most likely object underlying an image degraded by a known function and affected by Poisson distributed noise. In the following, section 3.3.2.1 describes in more detail the procedure for estimating the object. Section 3.3.2.2 illustrates the application of the Richardson-Lucy deconvolution method to estimate the underlying object from multiple subimages as in 
tomoSTED. Furthermore, the limitations of the method are discussed in section 3.3.2.3. Due to this limitations, possibilities to quantify the quality of the estimates in the case of simulated data are described in section 3.3.2.4.

\subsubsection{Estimation of the underlying object}

The Richardson-Lucy deconvolution method is based on Bayes' theorem and treats the object, the image as well as the degradation function or point-spread function as probability density functions. By iteratively generating estimates of the object, the likelihood of the estimates being the underlying object is increased and therefore the object is approximated.

Given an object $\mathrm{O}$ whose image $\mathrm{I}$ is degraded by a point-spread function PSF, then the image can be mathematically described by the convolution of the object with the point-spread function (see equation (37) or equation (2) in section 2.1] [65, 74]. For simplification, only one dimension is considered here. However, the considerations also apply to higher dimensions [65].

$$
\mathrm{I}(x)=\mathcal{P}(\mathrm{O}(x) * \operatorname{PSF}(x))
$$

In equation (37), $\mathcal{P}$ represents the Poisson distribution and considers the possible influence of noise on the image. Equation (37) raises the question whether the non-negative object can be recovered from the observed image if the point spread function is known and the noise is Poisson-like distributed. The probability $(\mathrm{P}(\mathrm{O}(\xi) \mid \mathrm{I}(x)))$, that the object exhibits a certain number of molecules at the position $\xi$ due to the observation of a specific intensity value in the image at position $\mathrm{x}$, can be described by means of Bayes's theorem [65, 74]:

$$
\mathrm{P}(\mathrm{O}(\xi) \mid \mathrm{I}(x))=\frac{\mathrm{P}(\mathrm{I}(x) \mid \mathrm{O}(\xi)) \mathrm{P}(\mathrm{O}(\xi))}{\left.\int \mathrm{P}(\mathrm{I}(x) \mid \mathrm{O}(\xi)) \mathrm{P}(\mathrm{O}(\xi))\right) d \xi} .
$$

In equation (38), $\mathrm{P}(\mathrm{I}(x) \mid \mathrm{O}(\xi)$ ) describes the probability that an object at the location $\xi$ contributes to the image at the position $\mathrm{x}$ and $\mathrm{P}(\mathrm{O}(\xi))$ represents the probability that there is an object point at the location $\xi$. Furthermore, $\mathrm{P}(\mathrm{O}(\xi))$ can be expressed via the law of total probability by means of the conditional probabilities $\mathrm{P}(\mathrm{O}(\xi) \mid \mathrm{I}(x))$ [65, 74]:

$$
\mathrm{P}(\mathrm{O}(\xi))=\int \mathrm{P}(\mathrm{I}(x)) \mathrm{P}(\mathrm{O}(\xi) \mid \mathrm{I}(x)) d x .
$$


Equation (39) can be further reformulated by substituting $\mathrm{P}(\mathrm{O}(\xi) \mid \mathrm{I}(x))$ with equation (38) leading to

$$
\mathrm{P}(\mathrm{O}(\xi))=\int \mathrm{P}(\mathrm{I}(x)) \frac{\mathrm{P}(\mathrm{I}(x) \mid \mathrm{O}(\xi)) \mathrm{P}(\mathrm{O}(\xi))}{\left.\int \mathrm{P}(\mathrm{I}(x) \mid \mathrm{O}(\xi)) \mathrm{P}(\mathrm{O}(\xi))\right) d \xi} d x
$$

The transformation of an object point into the image is given by the point spread function PSF which is assumed to be known. Therefore, the probability $\mathrm{P}(\mathrm{I}(x) \mid \mathrm{O}(\xi))$ equals the probability that the PSF exhibits an intensity value at the position $(x-\xi)$ $(\mathrm{P}(\mathrm{PSF}(x-\xi)))^{9}$ With this, the denominator of equation 40 represents the convolution of the object with the PSF. The probability of an object-, an image- or a PSF-point present at a certain position can be expressed by its intensity value at this position divided by the total intensity. Hence, the probabilities can written as: $\mathrm{P}(\mathrm{O}(\xi))=\mathrm{O}(\xi) / \sum \mathrm{O}, \mathrm{P}(\mathrm{I}(x))=\mathrm{I}(x) / \sum \mathrm{I}$ and $\mathrm{P}(\mathrm{PSF}(x-\xi))=\mathrm{PSF}(x-\xi) / \sum \mathrm{PSF}$. In addition, the intensity is conserved between the image and the object. Thus, their total intensities are the same $\left(\sum \mathrm{I}=\sum \mathrm{O}\right)$ [74]. This leads to

$$
\mathrm{O}(\xi)=\mathrm{O}(\xi) \int \frac{\mathrm{I}(x)}{\mathrm{O}(x) * \operatorname{PSF}(x)} \operatorname{PSF}(x-\xi) d x .
$$

The integral term in equation (41) represents a correlation of the fraction with the PSF. Therefore, equation (41) can be additionally notated via the correlation operator $\star \mathbb{1}^{10}$

$$
\mathrm{O}(\xi)=\mathrm{O}(\xi) \cdot \frac{\mathrm{I}(\xi)}{\mathrm{O}(\xi) * \operatorname{PSF}(\xi)} \star \operatorname{PSF}(\xi)
$$

W.H. Richardson and L.B. Lucy suggested an iterative procedure to approach the object [65, 74]:

$$
\mathrm{O}_{j}(\xi)=\mathrm{O}_{j-1}(\xi) \cdot \frac{\mathrm{I}(\xi)}{\mathrm{O}_{j-1}(\xi) * \operatorname{PSF}(\xi)} \star \operatorname{PSF}(\xi)
$$

The iterative procedure is indicated with the index $\mathrm{j}$. Starting with an initial guess for the object $\hat{\mathrm{O}}_{0}$, an estimated image $\hat{\mathrm{I}}=\hat{\mathrm{O}}_{0} * \mathrm{PSF}$ is calculated. Afterwards this estimated image is compared to the real measured image leading to a correction term for the estimated object which is denominated by $\mathrm{F}$ :

$$
\hat{\mathrm{O}}_{j}(\xi)=\hat{\mathrm{O}}_{j-1}(\xi) \cdot \mathrm{F}_{j-1} \text {. }
$$

\footnotetext{
${ }^{9}$ This results from the shift invariance of the PSF (see section 2.1.2.

${ }^{10}$ Most common microscope PSF can be approximated by a symmetric function $(\operatorname{PSF}(-\xi)=\operatorname{PSF}(\xi)$ [58]. For symmetric functions, the correlation is equivalent to a convolution. Therefore, the correlation operator in equation 42 could also be replaced by a convolution operator.
} 
By iterative repeating this procedure, the likelihood of the estimated object being the underlying object is increased at each iteration step. Under noise free conditions, the estimation of the object is continuously improved and converges to the true object [15]. In the presence of noise, the performance is limited as described in section 3.3.2.3. Moreover, equation 42 preserves the non-negativity constraint of the object and conserves the total intensity [65].

\subsubsection{Estimation of the underlying object in tomoSTED}

In tomoSTED microscopy, the object is not only observed with one PSF exhibiting one high resolution direction, but with several PSFs with different high resolution directions:

$$
\mathrm{I}_{i}=\mathrm{O} * \mathrm{PSF}_{i}
$$

Thus, here, one needs to find the common object underlying all observed images. As in the previously presented procedure (see section 3.3.2.1), estimates are made for the common object and an estimated image is calculated for each individual orientation [59, 60, 66, 86]. Afterwards, the estimated images are compared with the real individual images $\left(I_{i}\right)$ leading to a correction term for each individual orientation. The final correction term is obtained by averaging the individual correction terms obtained for each orientation [59, 60, 66, 86]. The overall correction term obtained in this way is given by

$$
\mathrm{F}_{j}=\sum_{i=1}^{\mathrm{N}} \frac{\mathrm{I}_{i}}{\hat{\mathrm{O}}_{j-1} * \mathrm{PSF}_{i}} \star \mathrm{PSF}_{i}
$$

Here, the individual PSFs are normalised such that the sum over all PSFs equals to one (see equation (47)). In this way, the overall correction term represents a weighted average of all individual correction terms.

$$
\sum_{i=1}^{\mathrm{N}} \mathrm{PSF}_{i}=1
$$

The choice of the initial estimate is not significant as long as this is a smooth, nonnegative function [65].

\subsubsection{Limitations of the deconvolution algorithm}

In the noise-free case, the deconvolution algorithm converges monotonically towards the object[15]. Hence, under ideal conditions the similarity between the estimates and the 
object increases with each iteration step and just depends on the number of iterations. However, one drawback is that the convergence rate decreases per iteration step [15] Thus, the performance is limited by the number of feasible iteration steps.

Another limitation is noise. The major source of noise in confocal microscopy is shot noise [89]. Due to the quantum nature of light, the measured signal intensity varies statistically which can be described by a Poisson distribution [62, 89]. Noise can drive the estimates away from the 'true' object [15, 18]. Very similar images can be created by many different objects. Thus, noise can lead to estimate deviating from the 'true' object [15]. Furthermore, noise is enhanced within each iteration [15, 18]. Thus, in the presence of noise and depending on the signal to noise ratio, the similarity between the estimates and the object increases only initially [62]. Therefore, in the case of a known object (for example in simulations), the deconvolution procedure can be mapped by measuring the similarity between the estimates and the object and can be stopped at the point of maximum similarity [62].

\subsubsection{Similarity quantification in the case of a known object}

Several ways exist to quantify the similarity between two images. One possibility is to measure the distance between two images in terms of their intensity value differences per pixel which is commonly called the mean squared error as illustrated in equation 50 [18, 58, 62, 72, 75],12] Due to its frequent use [18, 58, 62, 72, 94], the mean squared error is employed within this thesis to quantify the similarity between the object and the

${ }^{11}$ There exists several ways to speed up the convergence. One possibility, which has the advantage to preserve the non-negativity constraint, is to include an exponent to the correction term [2, 94]:

$$
\hat{\mathrm{O}}_{j}(\xi)=\hat{\mathrm{O}}_{j-1}(\xi) \cdot \mathrm{F}_{j-1}^{q} .
$$

During the iteration process, the exponent is adapted via

$$
\mathrm{q}(j)=\exp \left(\frac{\left\|\nabla \hat{\mathrm{O}}_{j-1}\right\|}{\left\|\nabla \hat{\mathrm{O}}_{j-2}\right\|}\right)-\left(\frac{\left\|\nabla \hat{\mathrm{O}}_{2}\right\|}{\left\|\nabla \hat{\mathrm{O}}_{1}\right\|}\right) .
$$

Due to the uncertainty how the amplification of the noise behaves when using different adapted exponents and therefore to ensure the comparability between the method of classical STED and tomoSTED, only the standard Richardson-Lucy deconvolution was employed within this thesis.

${ }^{12}$ Other possibilities to quantify the estimation procedure is to use the Kullback-Leibler divergence [109] or the signal to noise ratio 6, 94]. A minimal Kullback-Leibler divergence corresponds to a good match between object and estimate [109. In the case of considering the signal to noise ratio, the algorithm would be stopped when the estimate exhibits the maximum signal to noise ratio [6, 94]. 
estimates within simulations.

$$
\operatorname{MSE}\left(I_{1}, I_{2}\right)=\sum\left(I_{1}-I_{2}\right)^{2}
$$

Two identical images lead to a mean squared error of zero. Furthermore, equation (50) can be transformed into [58, 75]:

$$
\operatorname{MSE}\left(I_{1}, I_{2}\right)=\sum I_{1}^{2}+\sum I_{2}^{2}-2 \underbrace{\sum I_{1} I_{2}}_{:=c c}
$$

The first two terms in equation (51) only depend on the single images and thus do not contain information about the similarity between the two images [58, 75]. Thus, it is sufficient to only consider the third term to quantify the similarity [58, 75] which represents the cross-correlation (cc) of the two images. However, different local intensities between the two images strongly influences the cross-correlation [9]. Thus, most commonly the normalised variant of the cross-correlation is used to measure the similarity between two images [9, 98]. The normalised cross-correlation is shown in equation (52) and becomes one for identical images.

$$
n c c\left(I_{1}, I_{2}\right)=\frac{\sum\left(\left(I_{1}-\overline{I_{1}}\right)\left(I_{2}-\overline{I_{2}}\right)\right)}{\sqrt{\sum\left(I_{1}-\overline{I_{1}}\right)^{2} \sum\left(I_{2}-\overline{I_{2}}\right)^{2}}}
$$

When looking at the normalised cross-correlation within the Richardson-Lucy deconvolution, it should increase continually under ideal (no noise-free) measurement conditions and should approach one. In the case of noise, the normalised cross-correlation should increase until it reaches a maximum value and then start to decrease again as the estimates becomes dissimilar to the object again due to the influence of noise. 


\section{ISM-assisted tomographic STED microscopy}

The objective of combining tomoSTED microscopy with image scanning microscopy (ISM) was to improve the performance of tomoSTED in terms of sample-gentleness. Hereby, it was aimed for reducing the light dose applied onto the sample while still achieving the same image quality as tomoSTED. As presented in section 2.3, the light dose acting on the sample in tomoSTED microscopy depends on the applied STED laser power and the total exposure time. Higher laser powers and longer total exposure times therefore lead to a higher light dose. However, a reduction of the STED laser power leads to a lower resolution for the same depletion pattern shape. Hence, reducing the laser power is not an option to achieve a lower light dose and otherwise identical resolution. Therefore, it was sought for a possibility to reduce the total exposure time. TomoSTED achieves a highly-resolved image in two dimensions by imaging the sample with several differently oriented one dimensional depletion patterns. The total exposure time in tomoSTED is therefore the product of the exposure time for imaging with each individual orientation and the number of orientations used. Reducing the exposure time of each individual orientation would lead to a decrease of signal and therefore to a lower image quality. Hence, the most direct way to decrease the light dose is to image with a lower number of pattern orientations. However, only reducing the number of pattern orientations would lead to a poorer isotropic resolution in two dimensions as illustrated in figure 4.1 and explained in the following.

\section{tomoSTED}

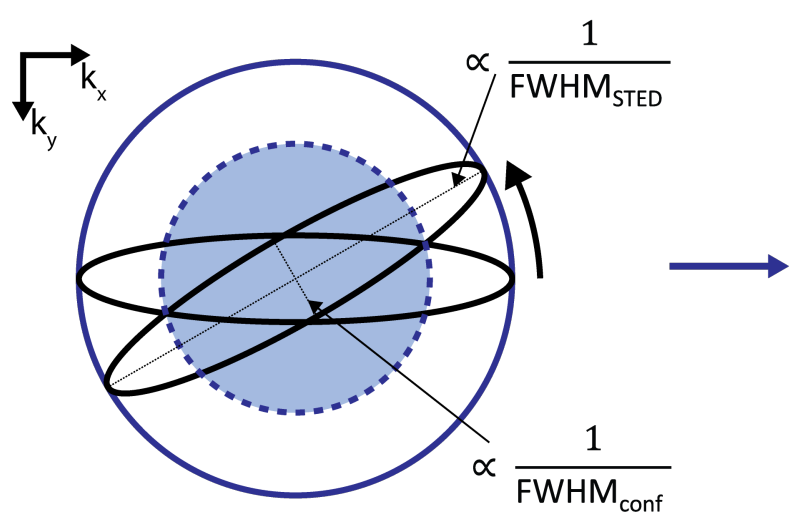

ISM-assisted tomoSTED

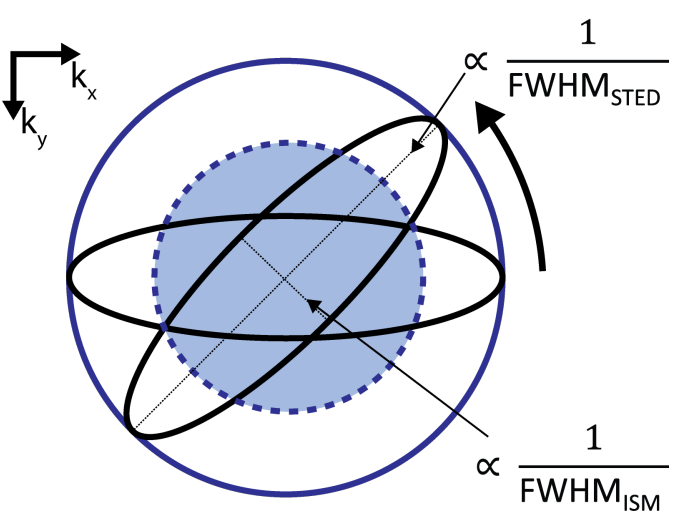

Figure 4.1: Schematically illustration of the coverage of spatial frequencies in two dimensions for tomoSTED and ISM-assisted tomoSTED. Due to the higher resolution in the confocal axis in the case of ISM-tomoSTED, the same area of spatial frequencies is covered with a lower number of pattern orientations

In figure 4.1, OTFs for individual orientations are schematically illustrated with black 
ellipses, which represent iso-lines at $50 \%$ of the maximum magnitude. When choosing an equal spacing between the individual orientations, the area of spatial frequencies, which is covered by all individual orientations, is exemplarily marked by the area of the blue dashed circle. If the number of orientations is decreased, which corresponds to a choice of a higher rotation angle (due to the equal spacing), the circular area of covered frequencies gets smaller. Hence, a lower isotropic resolution would be obtained. As observed in figure 4.1. the area of covered frequencies depends on the resolution in both the high-resolution direction (STED direction) as well as the diffraction-limited (confocal) direction. Thus, if the resolution in the confocal direction of tomoSTED could be increased, a larger area of covered spatial frequencies would be obtained when considering the same number of pattern orientations. Analogously, the number of pattern orientations could be reduced to obtain the same area of covered spatial frequencies and thus the same isotropic resolution in two dimensions (see figure 4.1). If otherwise the same measurement parameters (as for example exposure time, excitation and STED laser powers, scan pixel size, etc.) for the individual orientations are applied, a lower light dose could be achieved.

As illustrated in section 2.1, the resolution in a conventional confocal microscope can be improved by a factor of $\sqrt{2}$ by closing the confocal pinhole [105]. Unfortunately, reducing the pinhole size leads to a decrease of the detected signal and therefore a worse signal to noise ratio [13, 48]. This problem is solved in ISM, which was theoretically proposed in [87] and [5] and for the first time experimentally validated in [70]. ISM allows to improve the spatial resolution of a confocal microscope with an open pinhole by the factor of $\sqrt{2}$ while maintaining the signal [13, 70]. Therefore, ISM was chosen here to increase the resolution in the diffraction limited direction of the individual 1D STED PSFs and was investigated with respect to a reduction of the number of pattern orientations.

In the following, the idea and the method of ISM is described in section 4.1. Based on this, the subsequent section 4.2 discusses the appliance of the ISM principle to tomoSTED, including a validation on simulated and experimental data. In addition, the number of pattern orientations for ISM-tomoSTED, which leads to at least the same image quality as tomoSTED, is examined in section 4.3. Furthermore, the performance of ISM-tomoSTED in comparison to tomoSTED is experimentally validated in section 4.4 . 


\subsection{Image formation in image scanning microscopy}

Image formation in ISM can be considered as a combination of two image formation modes, namely confocal microscopy and wide-field microscopy [84, 88, 101]. For the sake of simplicity, the imaging process is described here solely in one dimension, but can be extended without loss of generality to more dimensions. In a confocal microscope [88, 101], an illumination/ excitation spot is scanned over the sample. The response of the sample is measured for each scan position with a point detector. In the case of a confocal microscope with a completely opened pinhole, the resolution is therefore exclusively defined by the excitation spot. Thus, the PSF of a confocal microscope with open pinhole is given by the excitation $\mathrm{PSF}, \mathrm{PSF}_{\text {exc }}(x)$. In a wide-field microscope, the whole field of view is homogeneously illuminated and the light emitted from the sample is imaged onto a detector array. Thus, the resolution in this case is solely defined by the detection PSF, $\operatorname{PSF}_{\text {det }}(s)$ [84, 88, 101]. Combining these two image formation modes, the point detector of a conventional confocal microscope can be replaced by a detector array [70, 84, 87]. This principle of scanning and detection with a detector array is illustrated in figure 4.2 .
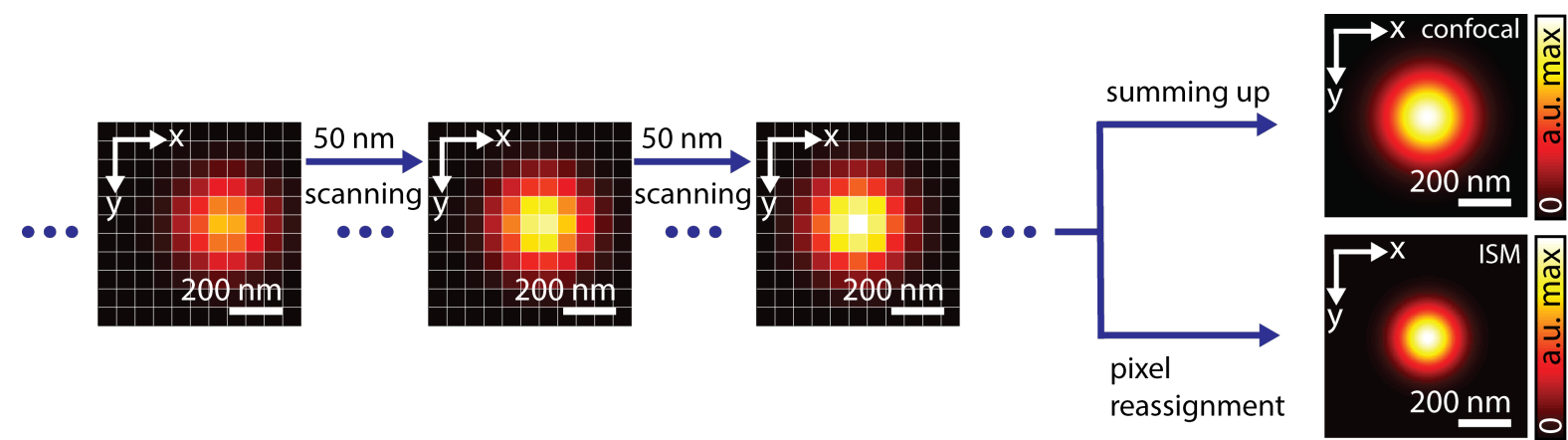

Figure 4.2: Illustration of a scanning microscope using a detector array to record the signal from the sample. By summing up the signal detected by all elements of the detector array for each scan position, a conventional confocal image is obtained. Considering each detector array element individually, a scanned image of the object is obtained for each detector array pixel. Reuniting these single scanned images during the pixel-reassignment procedure leads to an ISM image whose resolution is improved by a factor of $\sqrt{2}$. Assuming the same detector array size, the same signal contributes to the ISM image as to the conventional confocal microscope image. Thus, the signal of the ISM image is equal to the signal of the conventional confocal microscope image.

For each scan position, an image of the area around the excitation PSF is detected and the signal recorded by an individual pixel of the detector array at position $s$ is given by 84

$$
\operatorname{PSF}(x)=\operatorname{PSF}_{\mathrm{exc}}(x) \cdot \operatorname{PSF}_{\text {det }}(x-s) .
$$


Here, it is assumed that the sample is imaged with a magnification of one into the image space [84]. According to [84], this simplification does not restrict the generality. By summing up the signal collected by all detector array pixels for each individual scan position, a conventional confocal image is obtained which can be mathematically described by equation (54) [84, 87]. It represents the integral notation of the equations presented in section 2.1 for the PSF of a confocal microscope (see equation (3) and equation (4).

$$
\operatorname{PSF}_{\text {conf }}(x)=\int \operatorname{PSF}_{\text {exc }}(x) \cdot \operatorname{PSF}_{\text {det }}(x-s) D(s) \mathrm{d} s
$$

In equation (54), the confocal pinhole is included via $D(s)$, 84]

$$
D(s)= \begin{cases}1 & \text { for } s \leq a \\ 0 & \text { for } s>a\end{cases}
$$

where $a$ defines the size of the pinhole in relation to the sample space. The size of the confocal pinhole affects the volume within the sample which contributes to the final image. Therefore, it determines the microscope's optical sectioning capability and its resolution. Closing the pinhole leads to a resolution improvement of $\sqrt{2}$ as compared to a confocal microscope with completely opened pinhole (infinite large pinhole) [105]. Due to the resulting worse signal-to-noise ratio, a pinhole size of 1 Airy unit (AU) is usually used in conventional confocal microscopy [48].

In ISM, each detector array pixel is considered individually. If the size of the pixel is chosen small enough, it acts as a very small pinhole. When scanning an object, a scanned image is recorded by each pixel [84]. However, these scanned images are shifted relative to each other due to the relative shift of the individual pixels [88]. Therefore, a final image of the object can be obtained by a fusion of these images. This re-combination procedure is called pixel reassignment [13, 90]. During the pixel reassignment procedure, the images are shifted onto each other by considering the most probable shift due to the imaging process with an offset pinhole [76, 84]. This principle of image scanning microscopy is illustrated in figure 4.2 .

Theoretically, different approaches exist to determine the optimum magnitude by which the individual images have to be shifted. One possibility is to equalize the cut-offs of the OTFs of the excitation and detection PSF [90, 91]. Another option is to define the shift according to the widths of the excitation and detection PSF [76, 91]. In the following, 
the theoretical shift is analysed in real space, similar to [76]. For this purpose, the image of a point emitter is investigated, which is recorded by a pixel of the detector array at position $s$ (see equation (53)). As the point spread functions can be approximated by Gaussian functions [24, 110], an approximation for the optimum theoretical shift can be derived by inserting Gaussian functions into equation (53):

$$
\operatorname{PSF}(x)=A \cdot \exp \left(-\frac{x^{2}}{2 \sigma_{\mathrm{exc}}^{2}}\right) \exp \left(-\frac{(x-s)^{2}}{2 \sigma_{\mathrm{det}}^{2}}\right) .
$$

To determine the shift of $\operatorname{PSF}(x)$ in relation to $s$, the position of its maximum $x_{\max }$ is determined [92]. It can be obtained by setting the first derivative of equation (56) to zero. This leads to

$$
x_{\max }=\underbrace{\frac{\sigma_{\mathrm{exc}}^{2}}{\sigma_{\mathrm{exc}}^{2}+\sigma_{\mathrm{det}}^{2}}}_{:=m} \cdot s .
$$

According to equation (57), the position of the maximum is proportional to the pixel offset $s$ and the proportional factor $m$ solely depends on the standard deviation of the excitation and the detection PSF. Therefore, the factor $m$ can also be expressed via the PSF' FWHM:

$$
m:=\frac{\mathrm{FWHM}_{e x c}^{2}}{\mathrm{FWHM}_{\mathrm{exc}}^{2}+\mathrm{FWHM}_{\mathrm{det}}^{2}} .
$$

Thus, in order to recombine the scanned images obtained with the individual detector array pixels, each image has to be shifted by $-m s$ [88, 92], where $m$ is also called pixel reassignment factor. For excitation and detection PSFs of equal width, the reassignment factor $m$ equals 0.5 . This value is typically used in literature [70, 84]. However, if the optical conditions change $\left(\mathrm{FWHM}_{\mathrm{exc}} \neq \mathrm{FWHM}_{\mathrm{det}}\right)$ for example due to Stokes Shift or aberrations, the scaling factor differs [91]. Moreover, for Airy Patterns, the shift factor of equation (58) is only optimum for detector pixel offsets smaller than 1.335 AU [92, 93]. For larger array pixel offsets, the detection PSF becomes distorted and the strength of subsidiary maxima exceeds the strengths of the central maxima [92]. However, for detector array sizes smaller than 1.335 AU, the approximation of Gaussian functions leads to the same results as using Airy Patterns and is therefore within this range a suitable approximation.

In a next step, an analytical description of the final ISM PSF can be derived. For this purpose, $x$ can be substituted by $x=x+m s$ in equation (54) which corresponds to a shift of $-m s$ for each individual image, recorded by a detector array pixel. Afterwards, 
the reassigned images are summed up to obtain the final ISM image. For an infinitely extended detector array and a constant pixel reassignment factor $m$ [93], this results in

$$
\operatorname{PSF}_{\mathrm{ISM}}(x)=\int \mathrm{PSF}_{\mathrm{exc}}(x+m s) \cdot \mathrm{PSF}_{\operatorname{det}}(x-(1-m) s) \mathrm{ds} .
$$

Additionally, equation (59) shows that by choosing a pixel reassignment factor of $m=0$, a conventional confocal image is obtained, whereas $m=1$ leads to a conventional widefield image [88, 90, 92, 93]. An approximation of the ISM PSF can be obtained by assuming Gaussian functions for the excitation and the detection PSF again, leading to

$$
\operatorname{PSF}_{\mathrm{ISM}}(x)=A \cdot \sqrt{2 \pi} \cdot \frac{\sigma_{\mathrm{exc}} \sigma_{\mathrm{det}}}{\sigma_{\mathrm{ISM}}} \cdot \exp \left(-\frac{x^{2}}{2 \sigma_{\mathrm{ISM}}^{2}}\right) .
$$

Thus, for a Gaussian excitation and detection PSF, the final ISM PSF is again a Gaussian function with an effective standard deviation of $\sigma_{\mathrm{ISM}}^{2}=\sigma_{\operatorname{det}}^{2} m^{2}+\sigma_{\mathrm{exc}}^{2}(1-m)^{2}$. Hence, the width of the ISM PSF depends on the width of the excitation PSF, the width of the detection PSF as well as on the pixel reassignment factor chosen for the reconstruction:

$$
\mathrm{FWHM}_{\mathrm{ISM}}^{2}=\mathrm{FWHM}_{d e t}^{2} m^{2}+\mathrm{FWHM}_{\text {exc }}^{2}(1-m)^{2} .
$$

Hereby, the choice of the pixel reassignment factor $m$ according to equation (58) leads to an ISM PSF of minimal width and therefore to the highest resolution. As illustrated in equation (61), for an equal FWHM of excitation and detection PSF, the resolution in terms of FWHM can be laterally improved by a factor of $\sqrt{2}$ due to ISM compared to a confocal microscope with completely opened pinhole [76]. This resolution improvement corresponds to the theoretical resolution improvement of confocal microscopy with an infinitesimal small pinhole [105] (see section 2.1). However, the factor of $\sqrt{2}$ only holds true for an excitation and a detection PSF of equal width 90. If for example Stokes shift or aberrations are present, the improvement factor will be smaller [90].

Using equation (60), the amplitude of the ISM PSF can be compared with that of a conventional confocal microscope. By assuming Gaussian PSFs as well as an infinitely extended detector array, the following expression for the amplitude can be obtained via equation (54): $A_{\text {conf }}=A \cdot \sqrt{2 \pi} \sigma_{\text {det }}$. Thus, the ISM PSF of equation (60) can be expressed by the amplitude of the confocal PSF. This yields

$$
\operatorname{PSF}_{\mathrm{ISM}}(x)=A_{\mathrm{conf}} \cdot \mathrm{k}_{\mathrm{ISM}} \cdot \exp \left(-\frac{x^{2}}{2 \sigma_{\mathrm{ISM}}^{2}}\right) \text {, }
$$


where $\mathrm{k}_{\mathrm{ISM}}$ is defined by $\mathrm{k}_{\mathrm{ISM}}=\sigma_{\mathrm{exc}} / \sigma_{\mathrm{ISM}}$ which corresponds to the ratio of the width of the conventional confocal PSF to that of the ISM PSF. Thus, if the resolution of the ISM PSF is increased as compared to the conventional confocal PSF, the ISM PSF shows an amplitude which is enhanced by the factor of the resolution improvement due to ISM. However, as the same signal contributes to both the conventional confocal as well as the ISM image, the integral over both PSFs is identical. Thus, the increased amplitude of the ISM PSF results from the fact that the same signal is squeezed into a smaller peak leading to a higher amplitude [88]. This effect is called the superconcentration effect of ISM [77].

\subsection{Appliance of ISM to tomoSTED microscopy}

To improve the resolution in the diffraction-limited direction of all the individual 1D STED-PSFs, tomoSTED was combined with ISM. The realization is discussed in the following. For this, an overview of the procedure to obtain the final image in ISM-assisted tomoSTED is presented in section 4.2.1. Sections 4.2.2 - 4.2.5 focus on the pixel reassignment procedure in ISM-tomoSTED. For this, the pixel reassignment for ISM-tomoSTED is theoretically considered in section 4.2 .2 , whereas the practical implementation is discussed in section 4.2.3. Furthermore, the reassignment of ISM-tomoSTED images is validated in section 4.2 .4 via simulations and in section 4.2 .5 via experimental data. In addition, the employed ISM microscope has been calibrated such that the experimental data can be compared with the simulations (see section 4.2.6).

\subsubsection{Realization of ISM-assisted tomoSTED microscopy}

To apply ISM to tomoSTED microscopy, the point detector used in tomoSTED was replaced by a detector array. Thus, an ISM-measurement was performed for each differently oriented depletion pattern as illustrated in figure 4.3. Subsequently, each individual image was pixel reassigned. Finally, a in two dimensions highly-resolved image was generated by employing tomoSTED reconstruction methods (see section 3.3 to the individual ISM-tomoSTED images. As illustrated in section 3.3.1, the maximum-value reconstruction gives direct information about spatial frequencies present in the final images. Thus, only this reconstruction method was employed for validation. 


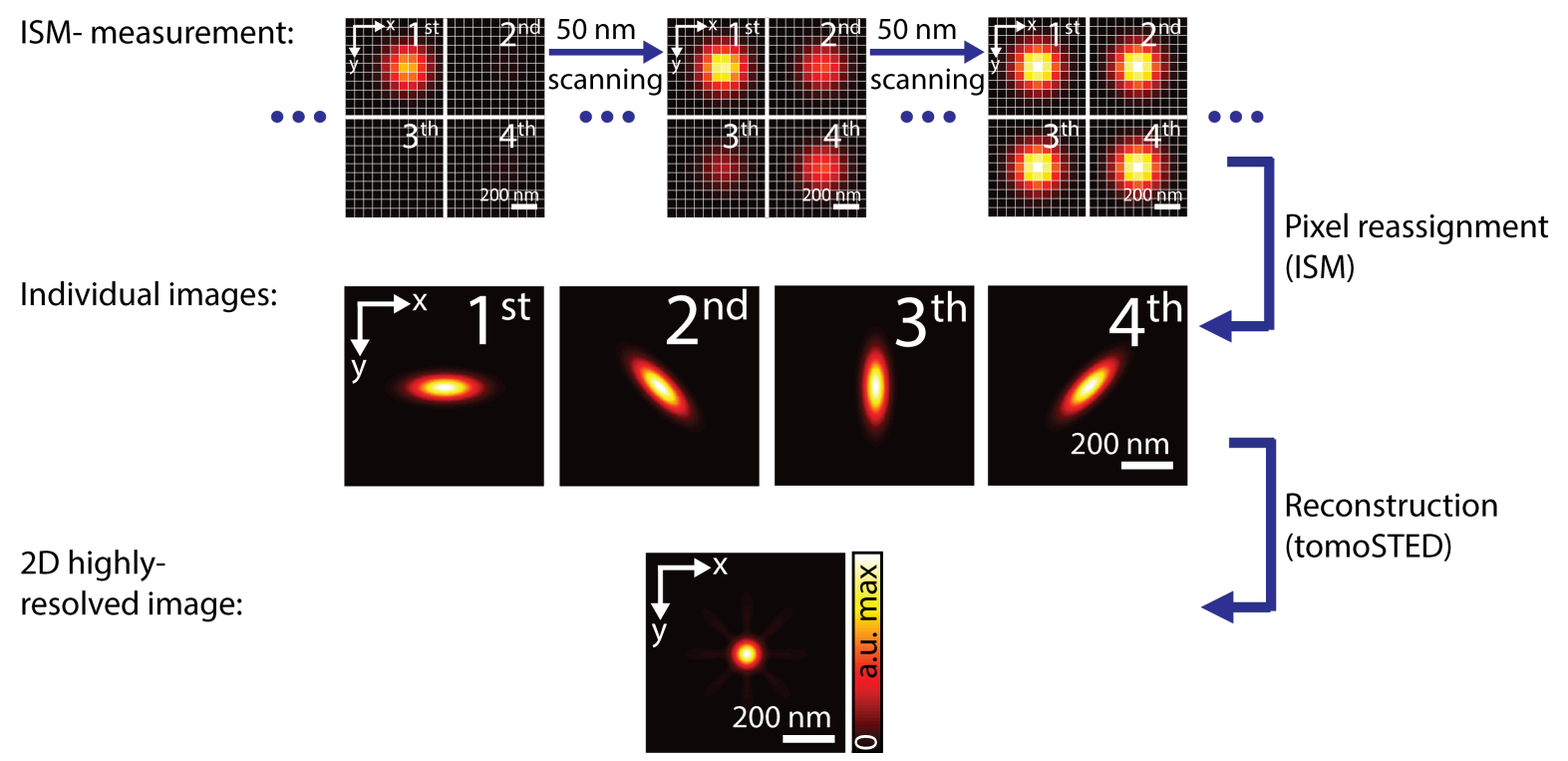

Figure 4.3: Illustration of the ISM-assisted tomoSTED microscopy operation principle: an ISM-measurement was performed for each differently oriented depletion pattern. Subsequently, each individual image was pixel reassigned. Finally, a in two dimensions highly-resolved image was generated by employing tomoSTED reconstruction methods to the individual ISMtomoSTED images.

\subsubsection{Theoretical consideration for reassigning ISM-tomoSTED images}

Based on the image formation process presented in section 4.1, the pixel reassignment factor for tomoSTED is discussed in the following. Individual tomoSTED PSFs exhibit a confocal and a high resolution direction and therefore they are asymmetrical. Hence, the image formation process, described in section 4.1, has to be expanded into two dimensions. Equation (59) accordingly yields

$$
\begin{aligned}
\operatorname{PSF}_{\mathrm{ISM}}(x, y)=\iint \mathrm{PSF}_{\mathrm{exc}}\left(x+m_{x} s_{x}, y+m_{y} s_{y}\right) \\
\cdot \mathrm{PSF}_{\operatorname{det}}\left(x-\left(1-m_{x}\right) s_{x}, y-\left(1-m_{y}\right) s_{y}\right) \mathrm{ds}_{x} \mathrm{ds}_{y} .
\end{aligned}
$$

In a first step, the ISM-PSF is considered for an effective tomoSTED excitation PSF with the confocal and STED direction aligned along either the $\mathrm{x}$ - or y-axis. As this PSF can be described approximately by Gaussian functions (see section 2.3), Gaussian functions are used to approximate the individual ISM-tomoSTED PSF obtained after 
pixel reassignment, leading to

$$
\operatorname{PSF}_{\operatorname{ISM}}(x, y)=A \iint e^{-\left(\frac{\left(x+m_{x} s_{x}\right)^{2}}{\sigma_{\text {exc, } \mathrm{x}}}+\frac{\left(y+m_{y} s_{y}\right)^{2}}{\sigma_{\mathrm{exc}, \mathrm{y}}^{2}}\right)} e^{-\left(\frac{\left(x-\left(1-m_{x}\right) s_{x}\right)^{2}}{\sigma_{\mathrm{det}, \mathrm{x}}^{2}}+\frac{\left(y-\left(1-m_{y}\right) s_{y}\right)^{2}}{\sigma_{\mathrm{det}, \mathrm{y}}^{2}}\right)} \mathrm{ds}_{x} \mathrm{ds}_{y} \text {. }
$$

The two integrations can be performed individually and thus, $\mathrm{PSF}_{\text {ISM }}$ in two dimensions is given by the product of the one-dimensional ISM-PSFs in the individual direction:

$$
\operatorname{PSF}_{\mathrm{ISM}}(x, y)=\operatorname{PSF}_{\mathrm{ISM}}(x) \cdot \operatorname{PSF}_{\mathrm{ISM}}(y)
$$

The optimum pixel reassignment factor for a one-dimensional PSF has already been derived in section 4.1 (see equation (58)). Thus, for each scan direction, a different pixel reassignment factor is obtained according to the width of the excitation PSF and detection PSF in this direction:

$$
m_{i}=\frac{\mathrm{FWHM}_{\mathrm{exc}, \mathrm{i}}^{2}}{\mathrm{FWHM}_{\mathrm{exc}, \mathrm{i}}^{2}+\mathrm{FWHM}_{\mathrm{det}, \mathrm{i}}^{2}}, \text { for } \quad i=x, y .
$$

Hence, if the confocal or the STED direction of the effective tomoSTED excitation PSF is aligned either along the $\mathrm{x}$ - or the $\mathrm{y}$-axis, the ISM-tomoSTED image can be obtained by shifting the individual detector array pixel images by $-m_{i} s_{i}$ according to the widths of the excitation and detection PSF in the $\mathrm{x}$ - and in $\mathrm{y}$-direction. Thus, a shift factor in relation to the confocal direction is obtained and one with respect to the highresolution direction. In this way, the single images recorded by the individual detector array pixels have to be shifted asymmetrically towards the optical axis. Extending these considerations to the case of arbitrary oriented tomoSTED excitation PSFs, the ISM images can be reconstructed in the same way, by applying the derived pixel reassignment factors (see equation (66) ) along the confocal and the high resolution direction.

\subsubsection{Practical realization of the reassignment of ISM-tomoSTED images}

In the previous section, the pixel reassignment for ISM-tomoSTED was investigated theoretically. Therefore, this section deals with its practical implementation.

Several ways exist to reconstruct the final ISM image. One possibility is to shift the signal recorded at each scan position by each detector array pixel by $-m_{i} s_{i}$ towards the optical axis, using the theoretical optimum pixel reassignment factor $m_{i}$. Subsequently, 
all signals registered at a respective scan position are summed up [70, 84]. Similar to this procedure, the individual ISM-tomoSTED image for different orientations of the 1D depletion pattern can be obtained by rotating each detector array image by the angle by which the confocal /STED direction is rotated from the $\mathrm{x}$-/y-axis. Afterwards, the signal from each pixel can be shifted asymmetrically towards the optical axis following equation (66). After having rotated the images back, the images can be registered at their respective scan positions and summed up. However, the disadvantage of this procedure is that it requires some prior calibration steps like the determination of the rotation angle, the widths of the excitation and the detection PSFs as well as the pixel size of the detector array with respect to the sample space. Furthermore, these a-priori calibrations might not be optimal if the widths of the excitation- or the detection PSF vary during a measurement for example due to varying aberrations [13].

Another possibility to recombine ISM-data is to use image registration methods in order to estimate the shift vectors directly from the measured raw data [12, 13, 14]. Most commonly, either the cross-correlation method [26, 29] or the phase correlation method [9, 61, 71] is utilised for the registration of displaced images. Considering mathematically two images which are translated by a vector $\left(x_{0}, y_{0}\right)$, the images can be described by the following relation $f_{2}(x, y)=f_{1}\left(x-x_{0}, y-y_{0}\right)$ [61, 71]. According to the Fourier shift theorem, the Fourier transforms of these translated images are related via a phase shift: $\mathcal{F}_{2}(u, v)=\mathcal{F}_{1}(u, v) e^{-i\left(u x_{0}+v y_{0}\right)}$ [61, 71]. Additionally, the cross power spectrum of these functions equals the phase shift:

$$
\frac{\mathcal{F}_{2}(u, v) \mathcal{F}_{1}(u, v)^{*}}{\left|\mathcal{F}_{2}(u, v) \mathcal{F}_{1}(u, v)^{*}\right|}=e^{-i\left(u x_{0}+v y_{0}\right)} .
$$

Moreover, the inverse Fourier transform represents a Delta-Dirac function at the location of the displacement [61, 71]:

$$
\mathcal{F}^{-1}\left(\frac{\mathcal{F}_{2}(u, v) \mathcal{F}_{1}(u, v)^{*}}{\left|\mathcal{F}_{2}(u, v) \mathcal{F}_{1}(u, v)^{*}\right|}\right)=\delta\left(x-x_{0}, y-y_{0}\right)
$$

Thus, the inverse Fourier transform of a cross-power spectrum of two translated images results in a very sharp peak at the location of the displacement. Therefore, this method is used in the phase correlation method to determine the shift between two images [61]. Furthermore, it is evident from equation (67) that the cross-power spectrum represents a normalised form of the standard cross-correlation. Thus, in theory, the phase correlation method provides a sharper correlation peak at the displacement point than the standard 
cross-correlation and is hence considered to be more accurate [25]. Moreover, the phase correlation method was demonstrated to be more robust against differing conditions between two images like for example varying illumination or a differing detection sensitivity [61]. Hence, the phase correlation method was used within this thesis to estimate the shift factors for the pixel reassignment process.

When the phase correlation method is used for pixel reassignment, the shift of each image recorded by an individual detector array pixel $\left(\mathrm{I}_{i, j}\right)$ to the image captured by the central pixel $\left(I_{s_{c_{1}}, s_{c_{2}}}\right)$ is determined [13, 14]. The central pixel is located on the optical axis and thus exhibits an overlapping excitation and detection PSF. Therefore, for each individual image, the inverse Fourier transform of the cross power spectrum with respect to the central image is calculated [13, 14]:

$$
r_{i, j}=\mathcal{F}^{-1}\left(\frac{\mathcal{F}\left(\mathrm{I}_{i, j}\right) \mathcal{F}\left(\mathrm{I}_{s_{c_{1}}, s_{c_{2}}}\right)^{*}}{\left|\mathcal{F}\left(\mathrm{I}_{i, j}\right) \mathcal{F}\left(\mathrm{I}_{s_{c_{1}}, s_{c_{2}}}\right)^{*}\right|}\right) .
$$

Theoretically, employing the phase correlation method leads to a Delta- Dirac peak at the image displacement point. In practice, however, the peak can be broader than the expected, due to the utilisation of discrete Fourier transform, noise within the images, the finite size of the images as well as subpixel shifts [25, 47]. In this case, the image shift can be estimated by determining the position of the maximum of the phase correlation peak [9, 47]. One way to determine the maximum is to use a Gaussian mask algorithm as presented in [13, 26]. Within this thesis, the Gaussian mask algorithm written by the authors of [23] was used to localize the peak of the phase correlation method.

In order to identify the central image, the detector pixel lying on the optical axis has to be determined first. The central pixel features an overlapping excitation and detection PSF and will therefore collect the most signal compared to all other pixels. In the realization of the image scanning microscope used within this thesis, the beam was descanned before reaching the detector. Thus, the central pixel was the same for all scan positions. It was determined by integrating the signal recorded by each pixel of the detector array and by fitting a two-dimensional Gaussian function to the integrated values. 


\subsubsection{Validation of the pixel reassignment of ISM-tomoSTED via simulated data}

TomoSTED and ISM-tomoSTED data were simulated to validate the pixel reassignment process for ISM-assisted tomoSTED via the phase correlation method. The simulations were performed using Gaussian shaped excitation and detection PSFs. The width of the Gaussian functions was chosen in accordance to the mean value of experimentally measured PSFs (see sections 4.2.6 and 4.2.5). Hence, an FWHM of $268 \mathrm{~nm}$ was assumed for the diffraction-limited and an FWHM of $70 \mathrm{~nm}$ for the high resolution direction of the effective excitation PSF. The detection PSF was considered to be symmetric with an FWHM of $282 \mathrm{~nm}$. Furthermore, the pixel size of the detector array was assumed to be $70 \mathrm{~nm}$ (see section 4.2.6). Exemplarily, simulation results for an orientation of the high resolution direction rotated by $0^{\circ}$ and by $45^{\circ}$ with respect to the $y$-axis are presented in figure 4.4 .

(a)
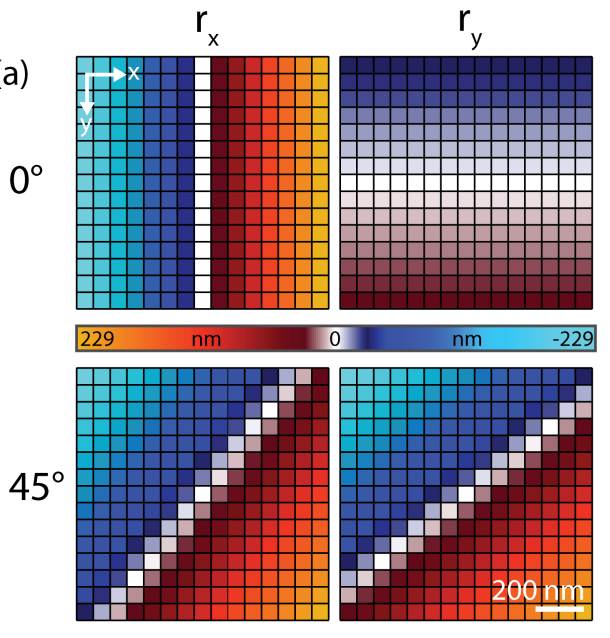
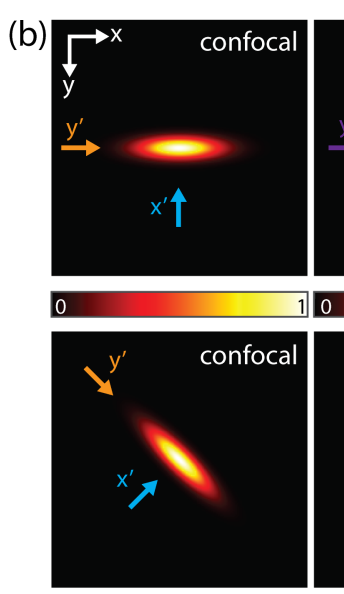
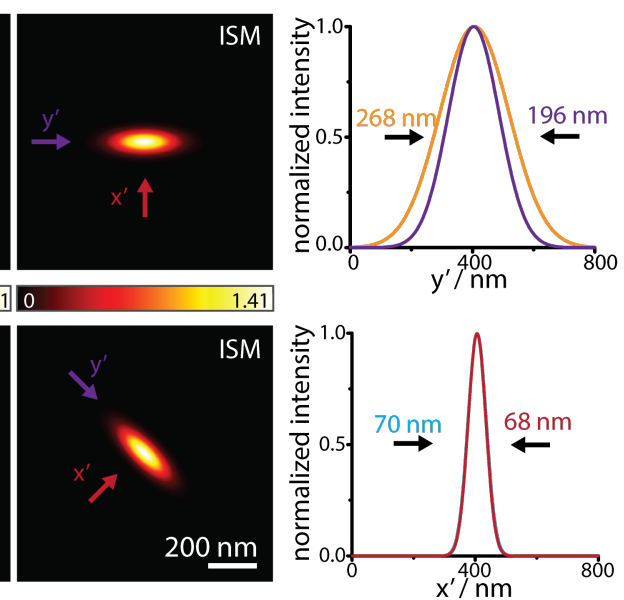

Figure 4.4: Validation of the phase correlation method for the pixel reassignment of ISMassisted tomoSTED via simulated data exemplified of the $0^{\circ}$ and $45^{\circ}$ tomoSTED orientation. (a) Estimated shift factors $\mathrm{r}$ for the $\mathrm{x}$ and $\mathrm{y}$ direction with respect to the respective detector array pixel and (b) comparison of the ISM-tomoSTED PSF with the tomoSTED PSF including line profiles along the high and low resolution axis for the $0^{\circ}$ orientation

Figure 4.4 (a) shows the shifts for the $\mathrm{x}$ and the $\mathrm{y}$ direction determined with the phase correlation method for the individual pixels of the detector array. They behave linearly in both the diffraction-limited as well as the high-resolution direction. Furthermore, the magnitude of the shifts in the diffraction-limited is higher than in the high-resolution direction of the effective excitation PSF as expected due to the dependence of the pixel reassignment factor on the width of the excitation and detection PSF (see equation (66)). 
They thus show the behaviour predicted in the theoretical discussion on the optimal pixel reassignment factor for ISM-tomoSTED (see section 4.2.2).

To validate the suitability of the determined shifts, they were compared to the theoretical expectation. Theoretically, the shift of an image, recorded by an individual detector array pixel, depends on the pixel reassignment factor $m_{i}$ and the pixel's distance from the optical axis $\left(m_{i} s_{i}\right)$ (see sections 4.1 and 4.2.2). Therefore, a pixel reassignment factor for the diffraction-limited direction and a respective factor for the high resolution direction can be calculated. For the $0^{\circ}$ orientation, a mean pixel reassignment factor of 0.468 was obtained along the diffraction limited direction and an average factor of 0.057 for the high resolution direction. Furthermore, a standard deviation of the factors of approximately $3 \cdot 10^{-5}$ was determined for the diffraction limited direction and of $10^{-6}$ for the high resolution direction. Hence, the deviation between the individual determined pixel reassignment factors is negligible. Based on the widths of the employed PSFs, a theoretical pixel reassignment factor of 0.475 is expected along the diffraction limited direction and a factor of 0.058 for the high resolution direction (see equation (66). Hence, the estimated factor for the diffraction limited direction differs relatively by approximately $1 \%$ from the theoretical expected value and the relative difference in the high resolution direction amounts to approximately $2 \%$.

In a next step, the resolution of the individual ISM-tomoSTED PSFs was investigated and compared to that of the corresponding tomoSTED PSFs (see figure 4.4 (b)). For this purpose, line profiles along the lines indicated by the arrows in figure 4.4 (b) were analysed. Therefore, the FWHMs of the profiles were quantified by means of a Gaussian fit. Line profiles drawn for the $0^{\circ}$ orientation are presented in figure 4.4 (b). The respective line profiles of the $45^{\circ}$ orientation show approximately the same FWHMs and are displayed in the appendix section $\mathrm{B}$ in figure B.1. Compared to the corresponding tomoSTED PSF, a clear resolution improvement in the diffraction-limited direction is obtained while the width in the high resolution direction is mainly maintained. Comparing the widths of the ISM-tomoSTED PSF with the theoretical width predicted by equation (61), the theoretical expected width was obtained along the high resolution direction. However, the width in the diffraction limited direction was about $2 \mathrm{~nm}$ wider.

To investigate the slight deviation of the pixel reassignment factor as well as of the resolution, ISM data for the $0^{\circ}$ orientation were simulated with decreasing the size of the detector array pixels. The resulting FWHM of the reassigned ISM images as well as 
the mean pixel reassignment factor in the diffraction-limited direction are presented in figure 4.5 (a).
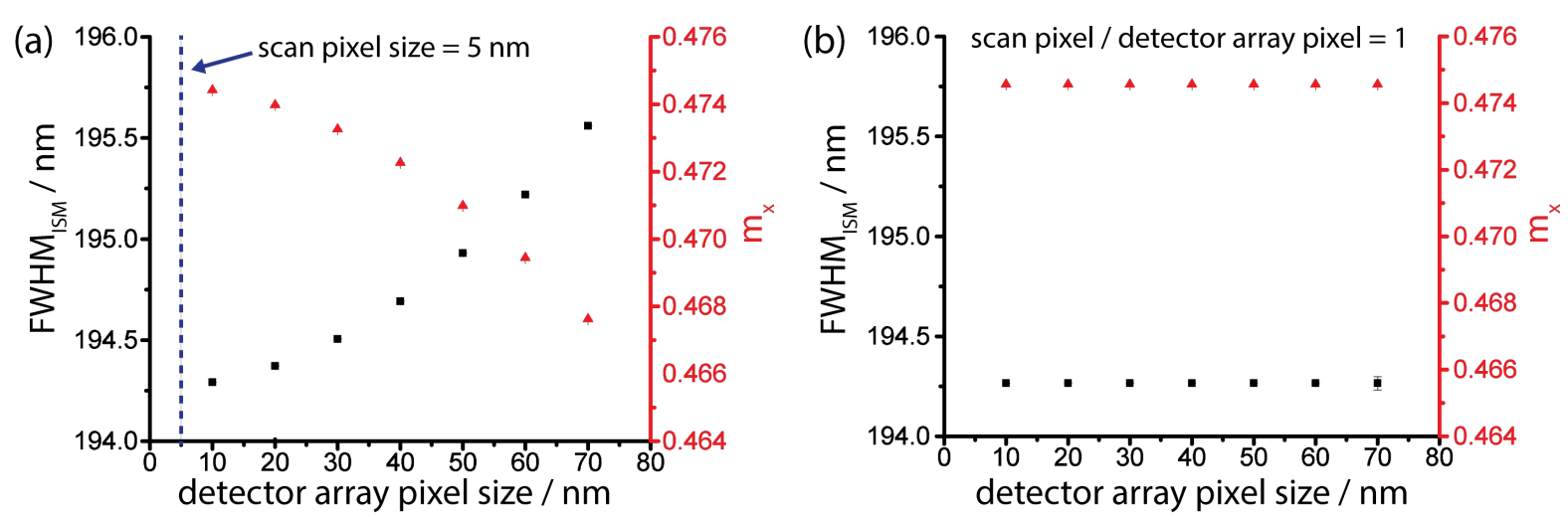

Figure 4.5: FWHM of the ISM PSF as well as the mean pixel reassignment factor as a function of the detector array pixel size for (a) the $0^{\circ}$ orientation along the diffraction limited direction using a scan pixel size of $5 \mathrm{~nm}$ and (b) the confocal excitation PSF employing an identical pixel size for scanning and for the detector array.

As shown in figure 4.5 (a), the FWHM of the ISM-tomoSTED PSFs in the diffraction limited direction decreases by reducing the size of the detector array elements. Approaching the case where the pixel size chosen for scanning equals the pixel size of the detector array, the width approximates the theoretical expected width of $194.3 \mathrm{~nm}$. A similar behaviour can be observed for the estimated pixel reassignment factor, it converges as well to the theoretical factor when coming closer to the case of equal pixel size selected for scanning and for the detector array. Therefore, the slight deviation can be explained by the applied pixelation.

Another reason for the deviations could be a too large chosen pixel size of the detector array. If the pixel size of the detector array is not small enough, the theoretical resolution improvement for the case of an infinitesimally small pinhole cannot be achieved. To exclude this possibility, data with different pixel size values were simulated, using an identical pixel size for scanning and for the detector array. As the influence of ISM on the resolution in the high-resolution direction is small, the confocal excitation PSF (268 nm) was used for these simulations. The results are presented in figure 4.5 (b) and show that all investigated cases lead to the theoretical expected values. Thus, the selected pixel size of $70 \mathrm{~nm}$ is small enough to achieve the theoretical resolution improvement of an infinitely small pinhole. Hence, the slight deviation resulted most likely due to the pixelation used for scanning and for the detector array. However, the deviations are only slight and the results presented in figure 4.5 are close to the theoretical expectations. 
Thus, the phase correlation method appears to work and seems to be suitable to reassign ISM-tomoSTED data.

\subsubsection{Experimental validation of the appliance of ISM to tomoSTED}

To validate ISM-tomoSTED experimentally, measured tomoSTED and ISM-tomoSTED PSFs were compared. To ensure the same measurement conditions for both methods, the measurements were carried out with the camera only. The sample used for this consisted of $48 \mathrm{~nm}$ fluorescent (crimson) microspheres (FluoSpheres carboxylate-modified microspheres, $48 \mathrm{~nm}$ diameter, crimson fluorescent (exc.: 625/ em.: 645); Life Technologies, USA). The fluorescent microspheres were immobilised on a cover slide surface coated with Poly-L-lysine $\left(0.1 \%(\mathrm{w} / \mathrm{v})\right.$ in $\mathrm{H}_{2} \mathrm{O}$, Sigma-Aldrich, USA), and were embedded in mowiol. Moreover, they were excited with an average laser power of $6.35 \mu \mathrm{W}$ and depleted with an average STED laser power of $94.4 \mathrm{~mW}$. The camera images were recorded with an exposure time of $150 \mu$ s and the scan step size was set to $20 \mathrm{~nm}$.

After having carried out the measurement, the individual tomoSTED and ISM-tomoSTED PSFs were reconstructed following the procedure described in section 4.1 (see figure 4.2). The individual tomoSTED images were obtained by registering the overall signal at the respective scan position and the individual ISM-tomoSTED images were reconstructed by pixel reassignment, as described in section 4.2.3. To ensure that the same signal is contributing to the final images of both methods, the same camera pixels were used. These pixels, representing the confocal pinhole, were chosen to cover a circular region around the central pixel exhibiting a diameter of $719 \mathrm{~nm}$. This diameter corresponds to about 1.1 AU with respect to a detection PSF featuring an FWHM of $282 \mathrm{~nm}$ (see section 4.2.6). Furthermore, a background correction was performed on the individual tomoSTED and ISM-tomoSTED images. For this purpose, the background was estimated from a square region of $820 \mathrm{~nm}$ length not containing any object and by determining its mean value. Exemplary tomoSTED and ISM-tomoSTED PSFs are displayed in figure 4.6 . 

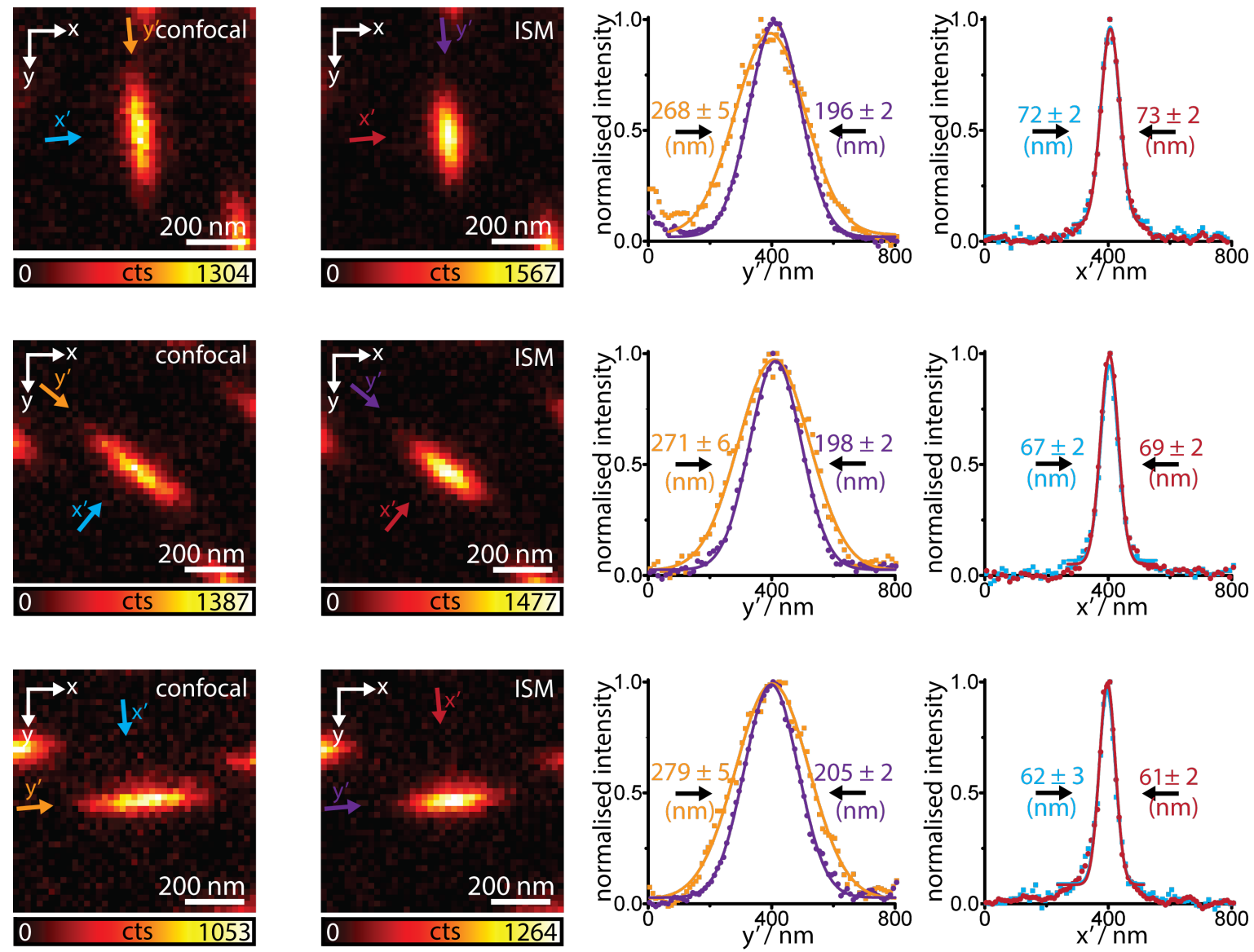

Figure 4.6: Application of the ISM-principle to asymmetric effective excitation PSFs. Examples of experimentally recorded microscope PSFs (measured on $48 \mathrm{~nm}$ fluorescent (crimson) microspheres) for different orientations of the high resolution direction with and without pixel reassignment and line profiles along the high and low resolution direction, marked with respective arrows.

In all images displayed in figure 4.6, a clear improvement of the width in the diffraction limited direction due to ISM is observable whereas the width in the high resolution direction is maintained. To quantify the width of the PSFs along the high and low resolution direction in more detail, profiles along the lines indicated with the arrows (coloured according to the respective direction) were drawn through the PSFs. In addition, the line profiles were averaged over three neighbouring lines. To determine the width of the line profiles a Gaussian fit was performed, presented in figure 4.6 with respective solid lines together with the experimental raw data marked with squares for tomoSTED and with dots for ISM-tomoSTED. By weighted averaging over five beads as well as over two orientations, namely the $0^{\circ}$-and $90^{\circ}$ orientation, per bead, a mean confocal width of FWHM $_{\text {tomoSTED, d.-l. }}=(268 \pm 1) \mathrm{nm}$ and a mean 
width of FWHM ISM-tomoSTED, d.-l. $=(201 \pm 1)$ nm in the ISM image along the diffractionlimited (d.-l.) direction was determined. Thus, the width was reduced on average by a factor of $1.33 \pm 0.01$. In the STED direction, a mean resolution of $(70 \pm 1) \mathrm{nm}$ was obtained for tomoSTED and $(70 \pm 1) \mathrm{nm}$ for ISM-tomoSTED. Thus, the ratio of the width in the STED direction amounts to $1.00 \pm 0.02$. When comparing these results with the simulations (see figure 4.4), it can be stated that the resolution improvement observed in the experiments is lower than expected by the simulations, where a resolution improvement of 1.37 was obtained along the diffraction limited direction, and an enhancement of 1.03 along the STED direction. For the STED direction, the results of the simulations lie within the three $\sigma$ range. Thus, the deviation for the factor in STED direction can be explained by statistical variations. The relative deviation between the experimentally measured resolution improvement for the diffraction limited direction and the simulations amounts to $3 \%$ and is slightly outside the three $\sigma$ range. This might be due to optical aberrations. An indication for existing aberrations was also observed when calibrating the detection PSF of the ISM microscope (see section 4.2.6). However, the deviations from the theoretical values are only slight and a clear improvement of the resolution in the diffraction limited direction can be observed. Thus ISM-tomoSTED has the potential to improve the performance of tomoSTED.

\subsubsection{Calibration of the ISM microscope}

Due to the use of the phase correlation method for the pixel reassignment (see section 4.2.3), a calibration of the microscope is not necessary. However, in order to be able to compare the experimental data with simulations, the magnification on the camera with respect to the sample plane and the width of the detection PSF have to determined. For this purpose, measurements on $48 \mathrm{~nm}$ fluorescent (crimson) microspheres, treated as described in section 4.2 .5 were carried out, using an average excitation laser power of $6.35 \mu \mathrm{W}$, an exposure time of the camera images of $1 \mathrm{~ms}$ and a scan step size of $20 \mathrm{~nm}$.

In order to calibrate the magnification in the detection path and therefore the size of the detector array pixels in sample space, the effect of scanning on the image generated on the camera is analysed in more detail. Due to the de-scanned detection, the excitation PSF stayed fixed with respect to the camera. Thus, while scanning over a point emitter, a movement of the emitter's image in the opposite direction to the scan direction can be observed on the camera. Thus, the pixel size of the camera in sample coordinates can be determined directly from the shift of the maximum intensity between two adjacent scan 
positions. Within this thesis, the position of the maximum intensity was obtained by fitting a two-dimensional Gaussian function to the camera image. To make the estimation more robust, not only two scanning steps were considered, but eleven, namely the scan position with the brightest image on the camera as well as the five preceding and the five following scan positions. In addition, not just one but five microspheres were used for this calibration. Figure 4.7 (a) shows the image of one of these microspheres together with the scan positions used, marked in red for the calibration in x-direction and in black for the one in y-direction. The positions of the maximum determined for the scan in x-direction are presented in figure 4.7 (c). Here, the dots and squares show the average values and the error bars the standard error of the mean value. In addition, the scan position where the position of the maximum coincides with the central pixel is defined as $0 \mathrm{~nm}$.

(a)

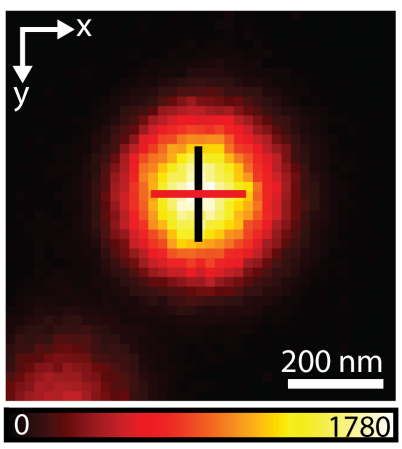

(b)

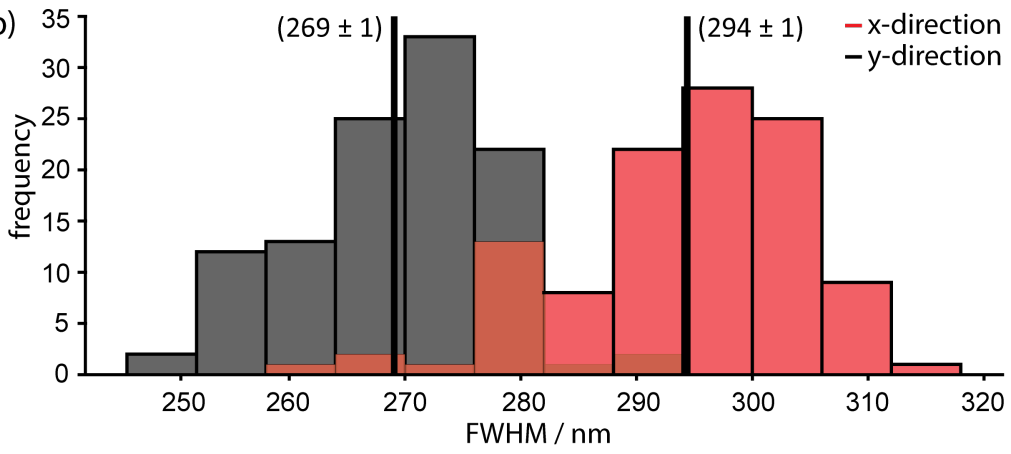

(c)

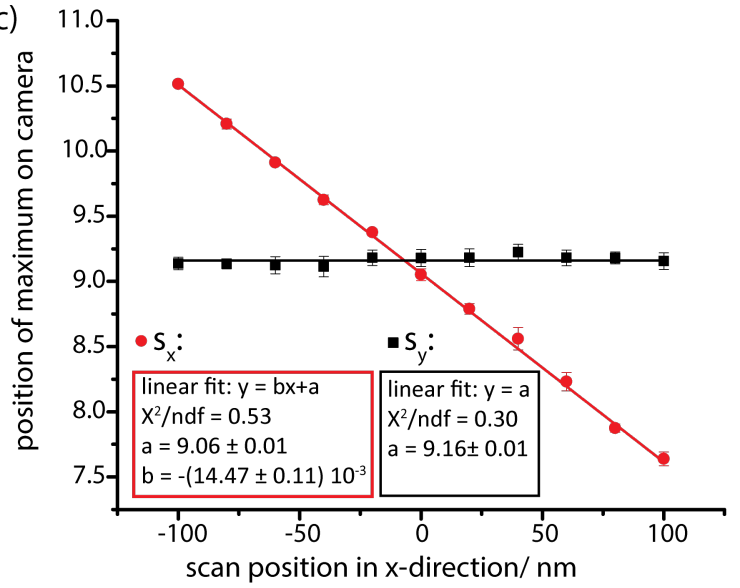

(d)

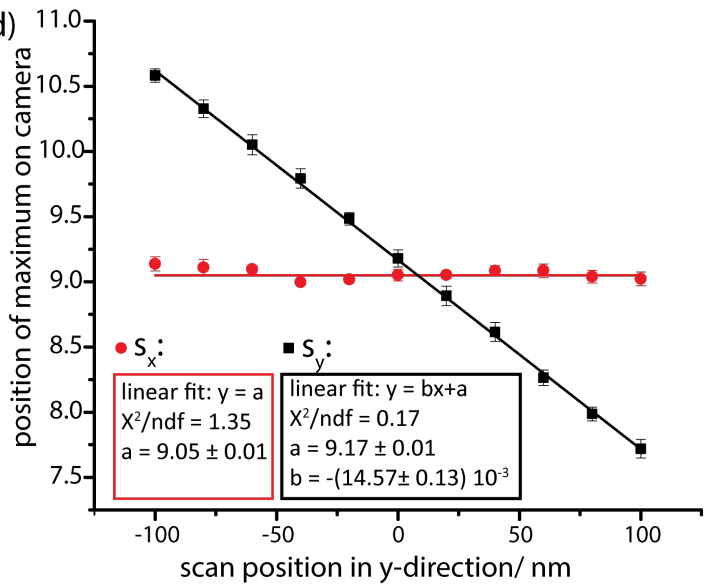

Figure 4.7: Calibration of the pixel size of the detector array in sample coordinates as well as the width of the detection PSF. (a) Exemplary image of a microsphere together with the scan positions used for the calibration, (b) determined FWHMs of the detection PSF, (c) linear regression to calibrate the pixel size in x-direction and (d) linear regression to calibrate the pixel size in y-direction. 
As shown in figure 4.7 (c), a linear shift of the position of the maximum in the scan position can be observed, while no shift can be recognised in the y-direction. The pixel size of the camera in sample can be determined can be determined by a linear regression, which resulted in a pixel size of about $(69 \pm 1) \mathrm{nm}$. The scan in y-direction led to the same qualitative result (see figure $4.7(\mathrm{~d})$ ) and the obtained pixel size agrees within the error range with that of the $\mathrm{x}$-direction. From the pixel size in sample coordinates and the physical camera pixel size of $6.5 \mu \mathrm{m}$ [3], the magnification of the microscope on the camera can be calculated leading to a factor of 94 , which agrees well with the theoretical magnification of 100 according to the used lenses (see section 3.1).

As at each scan position an image of the emitter is recorded, the width of the detection PSF was determined from the Gaussian fits performed at the selected scan positions (see figure 4.7 (a)). The result is presented in figure 4.7 (b). A FWHM of $(294 \pm 1) \mathrm{nm}$ was obtained in x-direction and $(269 \pm 1) \mathrm{nm}$ in $\mathrm{y}$-direction. A reason for this slight asymmetry might be optical aberrations. Since theoretically a symmetric detection PSF is expected, the average value of both directions of $282 \mathrm{~nm}$ was then used for the simulations.

\subsection{Number of pattern orientations for ISM-tomoSTED}

To improve the light dose of tomoSTED, ISM-tomoSTED was investigated with respect to the potential of reducing the number of required pattern orientations in order to achieve a nearly isotropic resolution. The number of required pattern orientations can be reduced if ISM-tomoSTED achieves at least the same isotropic resolution as tomoSTED using a lower number. Thus, final highly-resolved ISM-tomoSTED images were compared with those of tomoSTED, both obtained by the maximum-value reconstruction as described in section 3.3.1. The number of required pattern orientations were first analysed in Fourier space (see section 4.3.1.) and subsequently in real space (see section 4.3.2.

\subsubsection{Determination of the number of pattern orientations for ISM-tomoSTED in Fourier space}

In order to derive the number of required pattern orientations for ISM-tomoSTED in general, the coverage of spatial frequencies for both tomoSTED as well as ISM-tomoSTED microscopy was theoretically analysed (see section 4.3.1.1). Subsequently, the theoret- 
ical expectations were validated with both simulated and experimental data (see section 4.3.1.2.

\subsubsection{Theoretical derivation of the number of pattern orien- tations for ISM-tomoSTED}

To derive a theoretical description of tomoSTED and ISM-tomoSTED microscopy, the orientations of the individual tomographic PSFs were chosen in such a way that the angular spacing between them is equal. This means adjacent individual PSFs are rotated by the angle $\phi=\pi / \mathrm{N}$, where $\mathrm{N}$ describes the number of orientations used for the reconstruction. As both the individual tomoSTED and the individual ISM-tomoSTED PSFs can be approximated by Gaussian functions (see sections 2.3 and 4.2.2, they are considered to be Gaussian-shaped in the following. From this, it follows that the OTF of each individual PSF also has to be a Gaussian function. As an example, two OTFs rotated by the angle $\phi$ are schematically illustrated by black ellipses in figure 4.8. Here, the ellipses represent iso-lines at $50 \%$ of the OTFs' maximum magnitude.

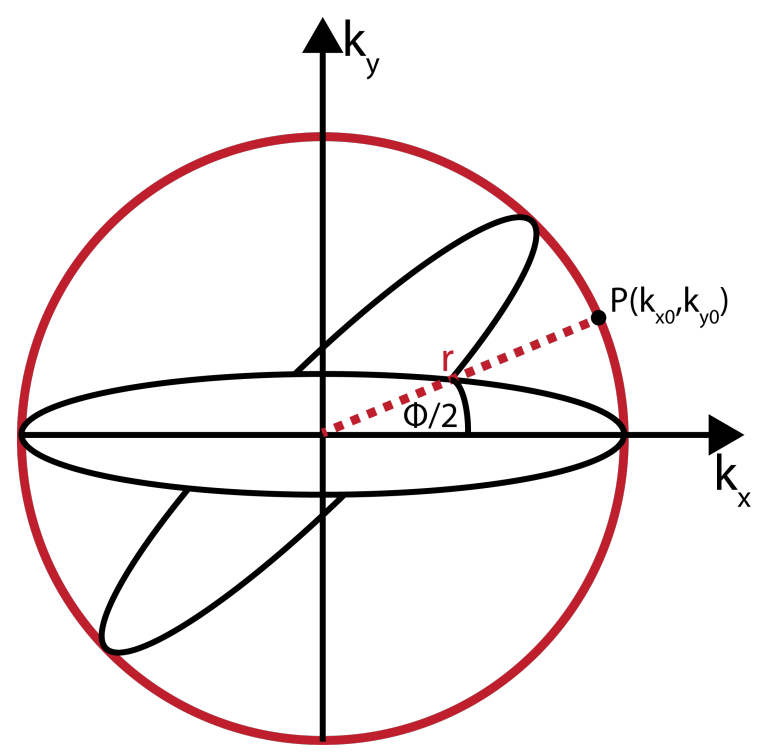

Figure 4.8: Illustration of the contribution of two individual tomoSTED PSFs rotated by an angle $\phi=\pi / \mathrm{N}$ to the final OTF retrieved by the maximum-value reconstruction.

To compare the coverage of spatial frequencies for both tomoSTED as well as ISMtomoSTED microscopy, the strength of the OTF for both methods along a circle at a specific distance from the centre, as illustrated in red in figure 4.8, was examined. A constant value along the circle corresponds to an isotropic coverage of spatial frequen- 
cies. To include all possible distances from the centre in these considerations, the radius $r$ is in the following defined to be proportional to the frequency at which the individual tomoSTED OTFs decayed along the high resolution direction to $50 \%$ of their maximum magnitude:

$$
r=a \cdot \frac{\mathrm{FWHM}_{k_{\mathrm{tomoSTED}, \mathrm{STED}}}}{2} .
$$

Here, $a$ denotes a proportional constant. For $a=1$, the radius is exemplarily illustrated in 4.1 with a red dashed line.

The strengths of the OTF in the minima along the circle represent the strongest deviations from a complete isotropic coverage of spatial frequencies and are therefore analysed in the following. The OTFs of the final highly-resolved images for both variants are considered to be reconstructed via the maximum-value reconstruction (see section 3.3.1). This means only the largest contribution from all individual images at a respective frequency in Fourier space is kept for the final image. Gaussian functions, assumed for the individual OTFs, decay strictly monotonically for values larger than the position of the maximum and are additionally symmetrical with respect to a reflection on their principal axes. Therefore, two individual OTFs rotated by the angle $\phi$ posses the same strengths at the angle $\phi / 2$. This position of equal strength between two differently oriented individual OTFs represents then the values of minimal magnitude of the final OTF. Thus, to analyse the minimal strength of the final OTF along the circle, it is sufficient to consider only one individual OTF and evaluate its magnitude at the angle $\phi / 2$. The position, at which the OTF's minimum is in the following analysed, is defined to be at the point $\mathrm{P}\left(k_{x 0}, k_{y 0}\right)$ (see figure 4.8 ).

To evaluate the OTF's strength in the point $\mathrm{P}\left(k_{x 0}, k_{y 0}\right)$, the individual OTF was chosen without loss of generality such that its high resolution direction points along the $k_{x^{-}}$ direction and its low resolution direction shows along the $k_{y}$-direction. Thus $\mathrm{FWHM}_{k_{x}}$ describes in the following the FWHM of the individual OTF in the $k_{x}$-direction and $\mathrm{FWHM}_{k_{y}}$ the respective FWHM in $k_{y}$-direction. The value of the OTF in the point $\mathrm{P}\left(k_{x 0}, k_{y 0}\right)$ is given by

$$
\operatorname{OTF}\left(\mathrm{k}_{x 0}, \mathrm{k}_{y 0}\right)=A \cdot \exp \left(-\frac{4 \ln (2)}{\mathrm{FWHM}_{k_{x}}^{2}} \mathrm{k}_{x 0}^{2}-\frac{4 \ln (2)}{\mathrm{FWHM}_{k_{y}}^{2}} \mathrm{k}_{y 0}^{2}\right) .
$$

Using polar coordinates, the coordinates $k_{x 0}$ and $k_{y 0}$ of the point $\mathrm{P}$ can be expressed by the angle $\phi / 2$ via the relations $\mathrm{k}_{x 0}=r \cdot \cos (\phi / 2)$ and $\mathrm{k}_{y 0}=r \cdot \sin (\phi / 2)$. Furthermore, 
the FWHM of the Fourier transform is related via $\mathrm{FWHM}_{k_{x}}=8 \ln (2) / \mathrm{FWHM}_{x}$ to the FWHM in real space. Expressing the FWHMs of the OTF by the FWHMs of the respective PSF as well as using equations (61) and (58), equation (71) can be transformed into

$$
\mathrm{OTF}_{i}\left(a, \mathrm{k}, \phi_{i}\right)=A \cdot \exp \left(-a^{2} \ln (2) \alpha_{i}-a^{2} \ln (2) \alpha_{i} \gamma_{i}\left(\mathrm{k}^{2}-1\right) \sin ^{2}\left(\frac{\phi_{i}}{2}\right)\right),
$$

where the respective microscope method is denominated by the index i. In equation 72 , $\alpha_{i}$ is given by

$$
\alpha_{i}= \begin{cases}1 & \text { for } i=\text { tomoSTED } \\ \frac{\beta^{2} k^{2}}{\beta^{2} k^{2}+1} & \text { for } i=\text { ISM-tomoSTED },\end{cases}
$$

where $\beta$ is defined as the ratio of the widths of the detection PSF to the excitation PSF of a normal confocal microscope

$$
\beta=\frac{\text { FWHM }_{\text {det }}}{\text { FWHM }_{\text {exc }}}
$$

In addition, $\mathrm{k}$ is the resolution increase factor of tomoSTED which is given by

$$
\mathrm{k}=\frac{\mathrm{FWHM}_{\text {tomoSTED, } \text { conf }}}{\mathrm{FWHM}_{\text {tomoSTED, STED }}} .
$$

Moreover, $\gamma_{i}$ in equation 72 is defined as

$$
\gamma_{i}= \begin{cases}1 & \text { for } i=\text { tomoSTED } \\ \frac{\beta^{2}}{\beta^{2}+1} & \text { for } i=\text { ISM-tomoSTED }\end{cases}
$$

Furthermore, the angle $\phi$ can be expressed via the number of pattern orientations $\phi=\pi / \mathrm{N}$, leading to

$$
\mathrm{OTF}_{i}\left(a, \mathrm{k}, \mathrm{N}_{i}\right)=A \cdot \exp \left(-a^{2} \ln (2) \alpha_{i}-a^{2} \ln (2) \alpha_{i} \gamma_{i}\left(\mathrm{k}^{2}-1\right) \sin ^{2}\left(\frac{\pi}{2 \mathrm{~N}_{i}}\right)\right) .
$$

The number of pattern orientations applied to ISM-tomoSTED should lead to the same strength in the OTF's minima as tomoSTED. Therefore, the number of pattern orientations for ISM-tomoSTED can be derived by equalising the magnitude of the OTFs in the minima:

$$
\mathrm{OTF}_{\text {ISM-tomoSTED }} \stackrel{!}{=} \mathrm{OTF}_{\text {tomoSTED }}
$$


Inserting equation $(77)$ into equation $(78)$ leads to

$$
\mathrm{N}_{\text {ISM-tomoSTED }}=\frac{\pi}{2} \frac{1}{\sin ^{-1}\left(\sqrt{\frac{\beta^{2}+1}{\beta^{4} k^{2}\left(k^{2}-1\right)}+\frac{\beta^{2} k^{2}+1}{\beta^{2} k^{2}} \frac{\beta^{2}+1}{\beta^{2}} \sin ^{2}\left(\frac{\pi}{2 \mathrm{~N}_{\text {tomoSTED }}}\right)}\right)} .
$$

In STED microscopy, resolutions of $20 \mathrm{~nm}-50 \mathrm{~nm}$ are mostly used [79], corresponding to resolution increase factors of about 13 - 5. For such high factors of resolution enhancement, the first summand below the square root of the equation 79 is approximately zero and the k-dependent part of the second summand tends towards one. Thus, for sufficiently high resolution enhancements, equation (79) can be approximated by

$$
N_{\text {ISM-tomoSTED }} \approx \frac{\pi}{2} \frac{1}{\sin ^{-1}\left(\sqrt{\frac{\beta^{2}+1}{\beta^{2}}} \sin \left(\frac{\pi}{2 \mathrm{~N}_{\text {tomoSTED }}}\right)\right)} .
$$

Equation (80) is illustrated in figure 4.9 using the widths of the excitation and detection PSFs calibrated experimentally in sections 4.2.5 and 4.2.6. Moreover, it represents a lower estimate for the number of pattern orientations. This means that the number of pattern orientations for ISM-tomoSTED determined according to equation (80) will always result in at least the same isotropic coverage of spatial frequencies as tomoSTED microscopy.

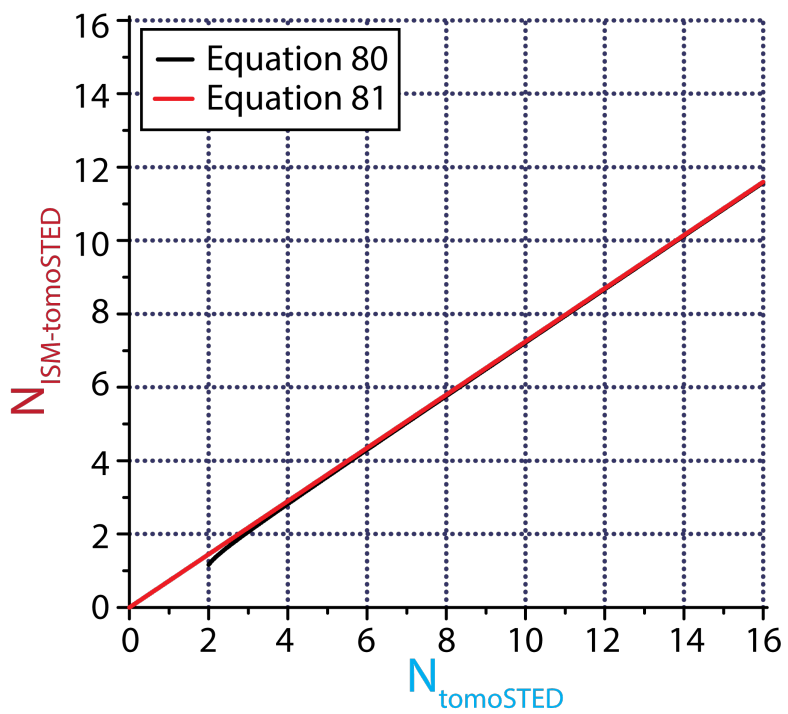

Figure 4.9: Illustration of the number of pattern orientations for ISM-tomoSTED. The black solid line represents the number of pattern orientations for ISM-tomoSTED according to equation 80 leading to at least the same strength in the minima of the OTF as tomoSTED. As shown with a red solid line, equation 80 can be approximated by a line though origin given by equation (81). 
As shown in figure 4.9, equation (80) approaches a line through origin already at a low number of tomoSTED pattern orientations. The slope of this straight line can be determined by the limit value of equation 80 for the case of a high number of tomoSTED pattern orientations $\left(\mathrm{N}_{\text {tomoSTED }} \rightarrow \infty\right)$. This leads to

$$
\mathrm{N}_{\text {ISM-tomoSTED }} \approx \sqrt{\frac{\beta^{2}}{\beta^{2}+1}} \cdot \mathrm{N}_{\text {tomoSTED }}
$$

In addition, the pixel reassignment factor $m$ in equation (61) can be expressed by $\beta$, leading to

$$
\mathrm{FWHM}_{\mathrm{ISM}}=\sqrt{\frac{\beta^{2}}{\beta^{2}+1}} \cdot \mathrm{FWHM}_{\mathrm{exc}} .
$$

By comparing equation (81) with equation 82 , it can be stated that the factor by which the number of pattern orientations can be reduced for ISM-tomoSTED corresponds to the resolution increase factor due to ISM in relation to the excitation PSF of the confocal microscope. Thus, the number of pattern orientations for ISM-tomoSTED can always be decreased by the factor of the resolution enhancement in the diffraction-limited direction of the 1D STED-PSFs. For the widths of the excitation and detection PSFs determined experimentally, the number of pattern orientations can be theoretically reduced by a factor of about 1.38 .

\subsubsection{Validation of the theoretical derivation via simulated and experimental data}

Simulated- as well as experimental data were used to validate the theoretical derived number of required pattern orientations for ISM-tomoSTED. Following the theoretical derivation presented in section 4.3.1.1, the strengths in the minima along radial profiles of tomoSTED- and ISM-tomoSTED OTFs were evaluated and compared. The radial profiles were drawn at a spatial frequency distance of $39.6 \mu \mathrm{m}^{-1}$ from the centre, corresponding to a magnitude decay of the individual tomoSTED OTFs' maximum to $50 \%$ along the high resolution direction. In figure 4.10 (a), tomoSTED and ISM-tomoSTED OTFs retrieved by the maximum value reconstruction with four and six pattern orientations, respectively, are shown. The circles along which the radial profiles were analysed are illustrated in blue for tomoSTED and in red for ISM-tomoSTED. In addition, it was investigated whether both the simulations and the experimental data can be theoretically well described by the approximation for high STED laser powers (see section 4.3.1.1). 
For sufficiently high STED laser powers, equation 77 can be approximated by

$$
\mathrm{OTF}_{i}\left(a, \mathrm{k}, \mathrm{N}_{i}\right) \approx A \cdot \exp \left(-a^{2} \ln (2)-a^{2} \ln (2)\left(\gamma_{i} \mathrm{k}^{2}-1\right) \sin ^{2}\left(\frac{\pi}{2 \mathrm{~N}_{i}}\right)\right) \text {. }
$$

The amplitude term in equation 83 , is identical for both tomoSTED and ISM-tomoSTED and can be therefore neglected. This leads to

$$
\mathrm{OTF}_{i}^{\prime}\left(a, \mathrm{k}, N_{i}\right) \approx \exp \left(-a^{2} \ln (2)\left(\gamma_{i} \mathrm{k}^{2}-1\right) \sin ^{2}\left(\frac{\pi}{2 \mathrm{~N}_{i}}\right)\right)
$$

Hence, the strength of the minima can also be investigated by normalising the radial profiles. Thus, normalised radial profiles are examined in the following. An example of normalised radial profiles for the reconstruction with four and six orientations is presented in figure 4.10 (b). It shows that the radial profiles contain minima corresponding to the number of orientations used for the reconstruction. Furthermore, the strength in the minima is higher for ISM-tomoSTED with the same number of pattern orientations applied as for tomoSTED. ISM-tomoSTED achieves approximately the same value in the minima when reducing the number of pattern orientations from six to four, resulting in at least the same isotropic coverage of spatial frequencies.

To compare the data with the theoretical model, namely equation 84 , the mean value of the OTF in the minima $<\mathrm{OTF}_{\text {min }}^{\prime}>$ was determined for several number of pattern orientations. For a FWHM of $70 \mathrm{~nm}$ of the 1D STED-PSFs in the high resolution direction, figure 4.10 (c) presents the via simulations obtained $\left\langle\mathrm{OTF}_{\min }^{\prime}>\right.$ together with the theoretical prediction in terms of equation (84). Equation (84) is displayed with solid lines and agrees well with the simulated data. Thus, a resolution of $70 \mathrm{~nm}$ along the STED direction can still be well described by the approximation for high STED laser powers. Therefore, estimating the number of orientations in terms of the equation (81) should also be well applicable for the resolution enhancement used here. In addition, higher values in the minima and therefore a better isotropic coverage of spatial frequencies for ISM-tomoSTED can be observed for any number of pattern orientations. 
(a)

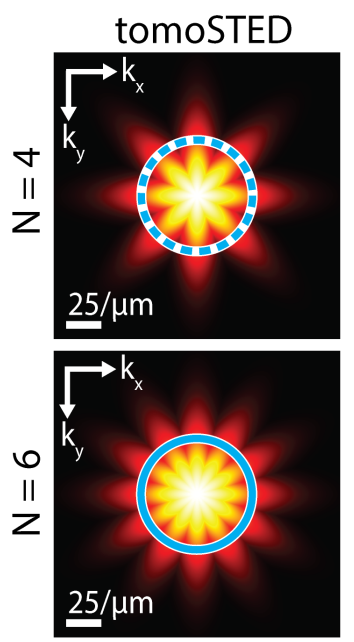

(c)

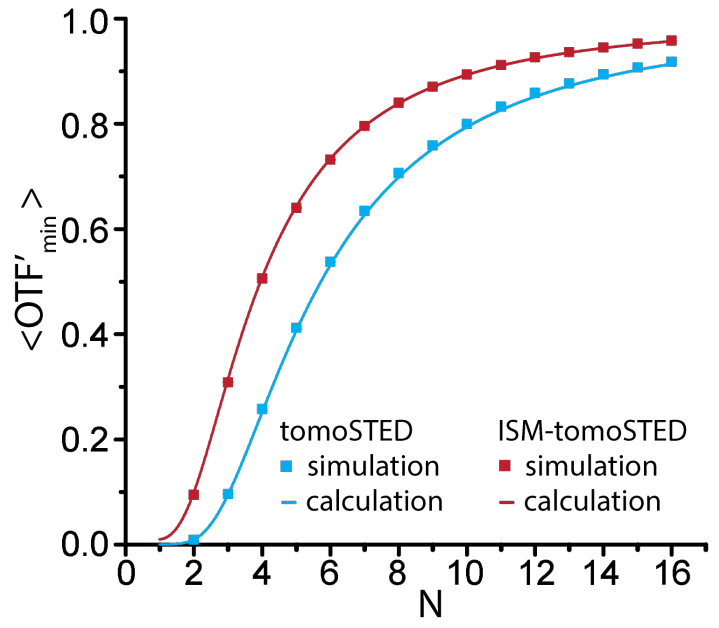

(b)

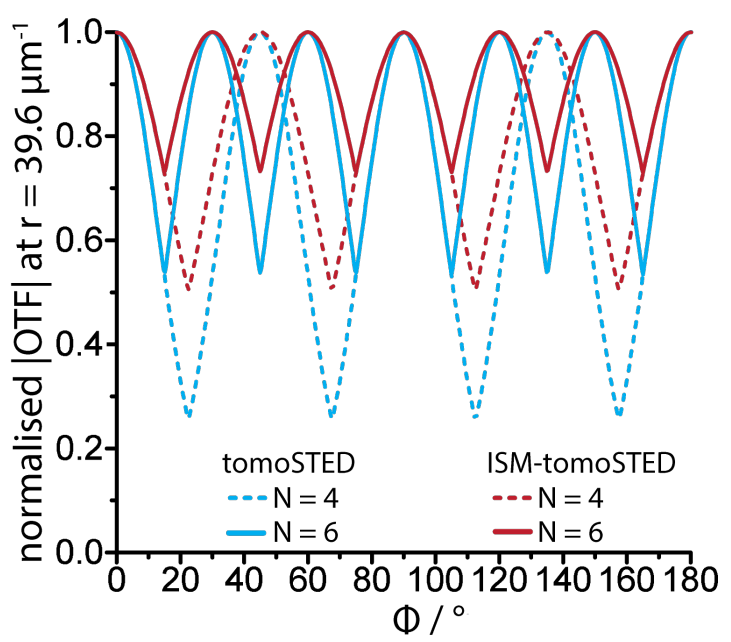

(d)

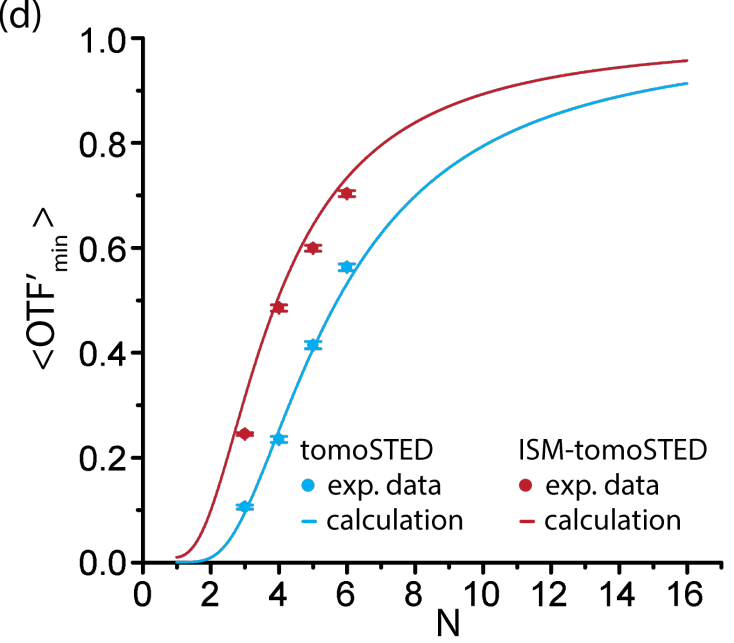

Figure 4.10: Analysis of tomoSTED as well as ISM-tomoSTED OTFs. (a) Magnitude of OTFs $\left(\mathrm{FWHM}_{\text {tomoSTED, STED }}=70 \mathrm{~nm}\right)$ for six and four pattern orientations with and without ISM. (b) Comparison of normalised radial profiles at a spatial frequency distance of $39.6 \mu \mathrm{m}^{-1}$ from the centre (indicated in blue and red in (a)) for four and six pattern orientations. (c) $\left\langle\mathrm{OTF}_{\min }^{\prime}\right\rangle$ as a function of the number of pattern orientations for simulated data as well as calculated according to equation (84). (d) Experimentally determined $\left\langle\mathrm{OTF}_{\min }^{\prime}\right\rangle$, derived from measurements on fluorescent microspheres, together with a curve accoding to equation (84).

To validate the theory as well as the simulations experimentally, $\left\langle\mathrm{OTF}_{\text {min }}^{\prime}>\right.$ was determined from measurements on fluorescent microspheres 13 . The results as a function of the number of pattern orientations are together with a curve according to equation (84) presented in figure 4.10 (d). It shows that the measured data follow with minor deviations the theoretical curve and thus agree with both the theory as well as the simulations. An explanation for the deviations might be the lower resolution increase due to ISM in

\footnotetext{
${ }^{13}$ The corresponding sample preparation is presented in section 4.2 .5 together with the employed imag-
} ing parameters. 
the experiments as compared to the theory (see section 4.2.5) as well as the limited sampling rate of the OTF due to the chosen scan pixel size of $20 \mathrm{~nm}$.

\subsubsection{Validation of the number of pattern orientations in real space via simulations with a ring-shaped object}

So far, the behaviour of ISM-tomoSTED has only been investigated in Fourier space. To validate whether the number of pattern orientations derived in Fourier space for ISMtomoSTED also leads to an at least equal isotropic resolution in all spatial directions (two dimensions) in real space, tomoSTED and ISM-tomoSTED data with an object consisting of five rings were simulated. The radii of the rings were chosen as $157.5 \mathrm{~nm}$, $241.5 \mathrm{~nm}, 337.5 \mathrm{~nm}, 445.5 \mathrm{~nm}$ and $565.5 \mathrm{~nm}$.

The previous analysis in Fourier space (see figure 4.9) has shown that the choice of four pattern orientations for ISM-tomoSTED should lead to the same resolution as for tomoSTED with six pattern orientations. Therefore, the simulations were performed with six and four pattern orientations. The final reconstructed images are presented in figure 4.11, revealing the ring structure of the object for all applied number of pattern orientations. Comparing the image for tomoSTED with six pattern orientations to that of ISM-tomoSTED with four pattern orientations, the inner rings appear visually better resolved for ISM-tomoSTED. To quantify the resolving capability in more detail, averaged radial profiles were analysed further. One possibility to quantify the resolution in real space is to examine if and how well two objects located at a known distance from each other can be separated in the image. Thus, the height of the minima was investigated. As expected, the height of the minima was highest for tomoSTED with four pattern orientations and lowest for ISM-tomoSTED with six pattern orientations, corresponding to the worst and best resolution, respectively. On average, approximately the same resolving capability was obtained for tomoSTED performed with six pattern orientations and for ISM-tomoSTED with four pattern orientations. Similar to the results of ISM-tomoSTED in Fourier space, the same isotropic resolution was achieved using a number of orientations reduced by a factor of 1.38 . 

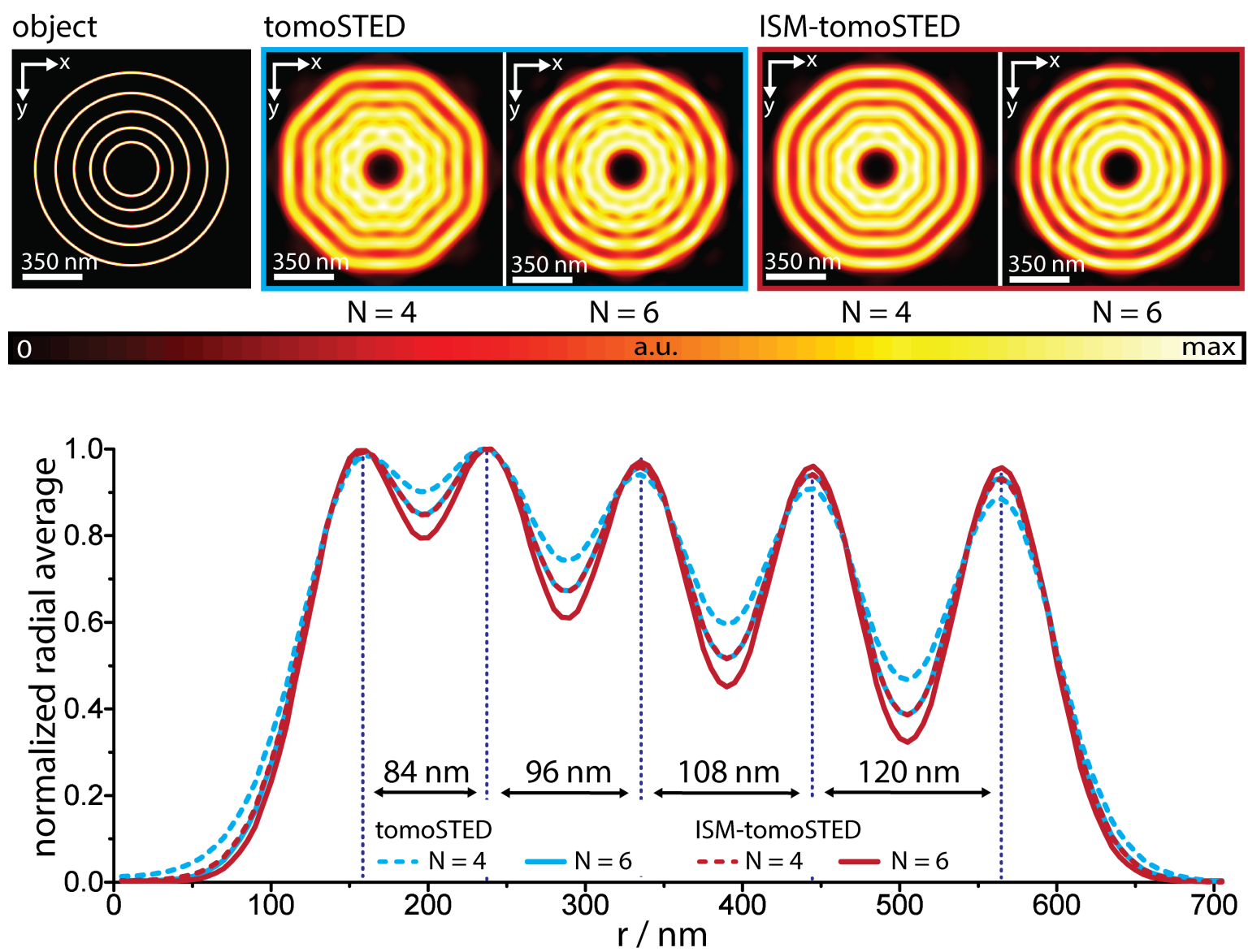

Figure 4.11: Comparison of the performance of tomoSTED and ISM-tomoSTED with six and four pattern orientations in real space by simulations. The radial average of the simulated images shows a comparable height of the minima and thus on average at least the same resolution, for tomoSTED with six pattern orientations and ISM-tomoSTED with four pattern orientations.

\subsection{Validation of ISM-tomoSTED on experimental data}

The behaviour of ISM-tomoSTED has so far been mainly validated by simulations. Thus, in the following, the performance of tomoSTED and ISM-assisted tomoSTED is examined experimentally by measuring $48 \mathrm{~nm}$ fluorescent microspheres and by imaging fixed vero cells. The results presented so far (see section 4.3.1 and section 4.3.2) showed that imaging with six orientations for tomoSTED and four orientations for ISM-tomoSTED leads to the same isotropic resolution. Therefore, this respective choice of number of pattern orientations for tomoSTED and ISM-tomoSTED is in the following experimentally examined.

To ensure the identical measurement conditions, tomoSTED and ISM-tomoSTED imag- 
ing was performed within the same experiment. Thus, fluorescent microspheres with a diameter of $48 \mathrm{~nm}^{14}$ were imaged with 12 orientations and the camera was used for detection. For the reconstruction, the orientations required for an equally spaced reconstruction with four and six orientations were selected from all measured orientations. Examples of image sections measured with four and six orientations are presented in figure 4.12 (a). To quantify the resolution, line profiles along the x-direction were analysed. In addition, these line profiles were averaged over three neighbouring lines. The width of these line profiles were determined via a Gaussian fit. Moreover, the analysis was performed on seven microspheres. For these seven microspheres, an average FWHM in $\mathrm{x}$-direction of $81.3 \pm 0.4 \mathrm{~nm}$ was obtained for tomoSTED with six orientations and a mean FWHM of $82.1 \pm 0.4 \mathrm{~nm}$ for ISM-tomoSTED with four pattern orientations. The average FWHMs agree with each other within the error range. Hence, the choice of six pattern orientations for tomoSTED and four pattern orientations for ISM-tomoSTED leads to approximately the same isotropic resolution. Examples of respective line profiles within the reconstructed images of the microspheres are presented in figure 4.12 (a) as well.

(a)
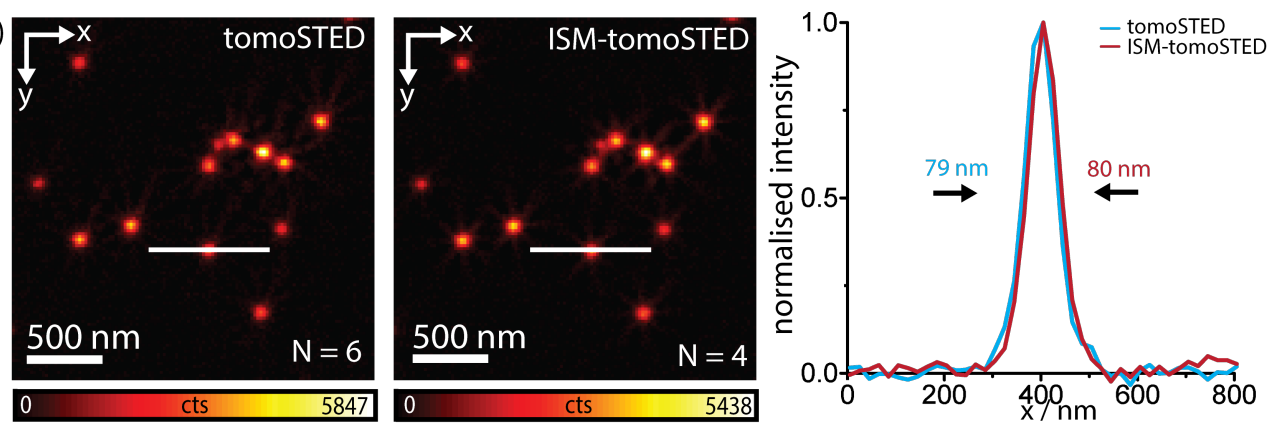

(b)
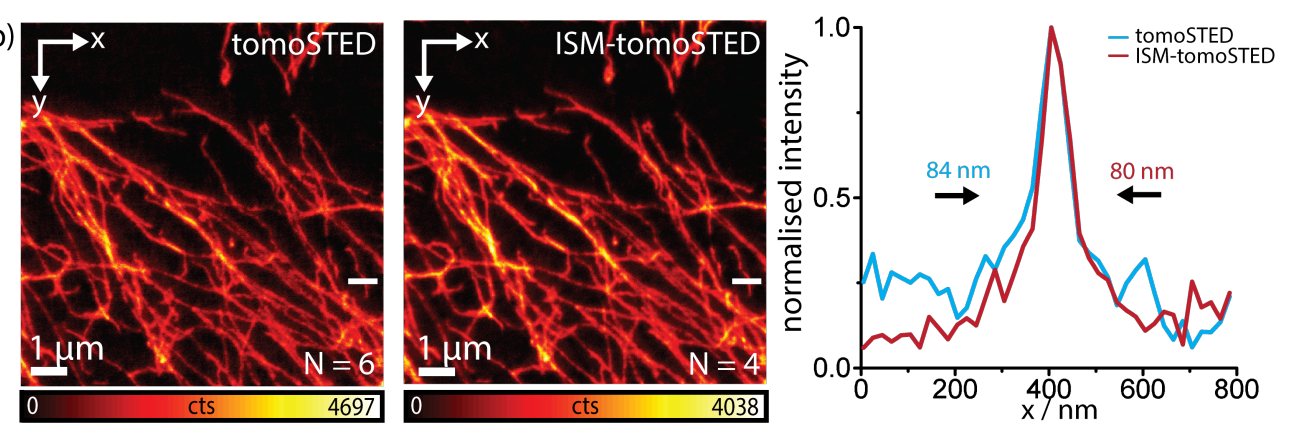

Figure 4.12: ISM-tomoSTED imaging with a reduced number of pattern orientations compared to tomoSTED for (a) $48 \mathrm{~nm}$ fluorescent microspheres and (b) fixed Vero cells.

\footnotetext{
${ }^{14}$ The corresponding sample preparation is presented in section 4.2 .5 together with the employed imaging parameters.
} 
To validate whether ISM-tomoSTED also achieves a comparable resolution in biological samples, tomoSTED and ISM-tomoSTED measurements were performed on methanolfixed Vero cells. After fixation, the fluorophore Abberior STAR 635P (Abberior GmbH, Germany) was attached via immunofluorescence staining to the $\alpha$-tubulin of the Vero cells (primary antibody: anti- $\alpha$-Tubulin-rabbit, Abcam, UK, secondary antibody: Abberior Star 635P anti-rabbit, Abberior, Germany). Afterwards, the sample was embedded in mowiol for imaging. An example of a Vero cell measured with six orientations for tomoSTED-microscopy and with four orientations for ISM-tomoSTED is presented in figure 4.12 (b). As previously, the cell was imaged with all orientations simultaneously. Here, 8 orientations, matching the orientations required for an equally spaced reconstruction with four and six orientations, were employed. An average excitation laser intensity of $7.01 \mu \mathrm{W}$, an average STED laser power of $162 \mathrm{~mW}$, together with a camera recoding time per scan pixel of $150 \mu$ s was used. Validating the resolution via line profiles through the filaments of the imaged Vero cells yielded a comparable resolution for both methods. One line profile is exemplarily presented in figure 4.12 (b). Therefore, it can be concluded that ISM-tomoSTED enables the imaging of biological samples with a reduced number of pattern orientations and thus allows to translate the resolution enhancement due to ISM into a lower light dose. 


\section{Three-dimensional tomographic STED microscopy}

Most biological samples are three-dimensional. Thus, the two-dimensional tomoSTED (2D tomoSTED) method has to be further developed into a three-dimensional application. Therefore, the aim of this thesis was to obtain a three-dimensional imaging method that works with a lower light dose while achieving at least the same image quality as the conventional variant of $3 \mathrm{D} \mathrm{STED}^{15}$. As illustrated in section 2.3 , a lower light dose for $2 \mathrm{D}$ tomoSTED could be achieved by rotating one dimensional depletion patterns in two dimensions [59, 60]. Therefore, it was expected that a lower light dose in three dimensions can be obtained by a rotation of one dimensional depletion patterns in three dimensions.

To be able to rotate one-dimensional depletion patterns in three dimensions, suitable phase masks were necessary (see section 5.1). In the following sections 5.2 and 5.3 , the individual 3D tomoSTED orientations created with the phase masks found are examined with regard to their potential in terms of a lower light dose. Thus, possible reduction factors for the laser power as well as for the exposure time are derived.

To get high resolution information in all spatial directions, the individual tomoSTED orientations have to be assembled to a final highly-resolved image. A continuous rotation of one-dimensional depletion pattern is unfavorable with respect to the light dose (see section 2.3). Thus, a finite number of orientations had to be selected (see section 5.4). The selected orientations were afterwards reconstructed by the reconstruction methods presented in section 3.3 which were found to be suitable for reconstructing final $2 \mathrm{D}$ tomoSTED images [59, 60]. This laid the foundation for the comparison between 3D tomoSTED and the conventional 3D STED (shown in section 5.5 for the maximum-value reconstruction and in section 5.6 for the Richardson-Lucy deconvolution).

\subsection{Phase masks for the rotation in three dimensions}

To derive suitable phase masks for the rotation in three dimensions, the intensity distribution within the focal region, generated by a microscope's objective lens, was considered.

\footnotetext{
${ }^{15}$ Same image quality corresponds to the same resolution in all spatial directions as well as an equal signal strength of the high spatial frequencies. For a more detailed description of the conventional $3 \mathrm{D}$ STED, please refer to section 2.2
} 
The experimental realization of tomoSTED as well as of the conventional STED variant within the framework of this thesis (see section 3.1) was designed for objective lenses with high numerical apertures. Thus, imaging with high numerical aperture objectives lenses (numerical aperture larger than $0.7([28]$ p.145)) is regarded in the following, which is described in section 5.1.1. In section 5.1.2, potential phase masks are derived from the intensity distribution obtained in section 5.1.1. Furthermore, the suitability of these phase masks is examined in section 5.1.3.

\subsubsection{Intensity distribution within the focal region for high numerical aperture objective lenses}

To get an approximation for the intensity distribution within the focal region, the focussing process of a high numerical aperture objective lens has to be considered. Here, the optical system is assumed to be aplanatic. In an aplanatic system, the optical system is axially stigmatic and obeys the sine condition [73]. Hence, a perfect image of an object, placed in a small region near by the optical axis, can be obtained by any pencil of rays with any angular divergence ([7], p.179-180), ([28], p.153-154). For an aplanatic system which images a point source placed at infinity, the electromagnetic field and therefore the intensity within the focal region can be described by the superposition of plane waves propagating from each point of the objective lens exit pupil towards the focal point [73]. This is explained in the following in more detail.

A monochromatic linear polarised plane wave is assumed which enters and completely fills the entrance pupil of the objective lens [24, 38, 73]. The plane wave is polarised at an angle $\phi_{0}$ with respect to the x-axis. The amplitude of this plane wave, in relation to the $\mathrm{x}, \mathrm{y}$-position within the entrance pupil can be described by [24, 38]

$$
\vec{E}(x, y)=E_{0}\left(\begin{array}{c}
\cos \left(\phi_{0}\right) \\
\sin \left(\phi_{0}\right)
\end{array}\right) P(x, y) \quad \text {,where } P(x, y)=e^{i \tilde{p}(x, y)}
$$

In equation $85, E_{0}$ represents the amplitude of the electric field and the function $\mathrm{P}(\mathrm{x}, \mathrm{y})$ accounts for phase shifts in the wavefront of the plane wave [24]. Thus, $\tilde{p}(x, y)$ represents a phase mask that can be applied to a beam and that leads to a spatially phase-shifted wavefront. In [50, 51], an optimisation algorithm was developed to find phase masks for STED microscopy that lead to the most efficient fluorescence inhibition. As a result of this optimisation algorithm, the, so-called, axial phase mask was identified as optimal 
for a resolution enhancement along the z-direction (see 2.2 section) and the, so-called 1D phase mask, as used for 2D tomoSTED (see section 2.3), was presented to be optimal for a resolution enhancement along the y-direction [50, 51]. All these phase masks, which were identified as optimal for a resolution increase in one dimension, consist only of phase shift values of 0 or $\pi$. Therefore, only phase shift values of 0 or $\pi$ were considered for $3 \mathrm{D}$ tomoSTED. Thus, depending on the position within the entrance pupil, $\mathrm{P}(\mathrm{x}, \mathrm{y})$ could only be 1 or -1 .

When the plane wave passes the objective lens, its plane wavefront is converted into a spherical wavefront due to refraction [38]. Moreover, as the electric field of an electromagnetic wave points perpendicular to the direction of propagation, it has to be tangential to the spherical wavefront after the objective [38]. Hence, the focussing by the objective lens leads to a change of the polarisation of the incoming wave. This transformation process is illustrated in figure 5.1, where the wavefronts are schematically indicated in blue.

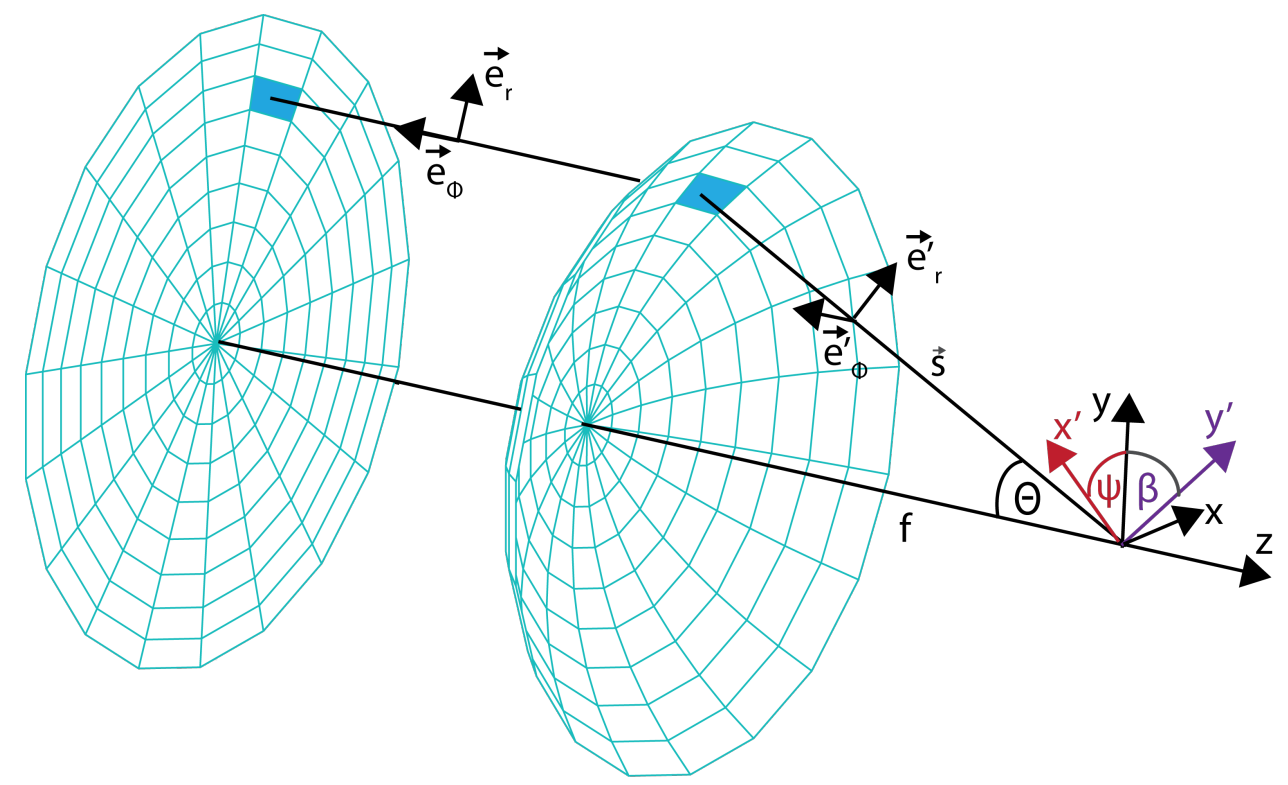

Figure 5.1: Schematic illustration of the focussing process of a microscopes objective lens, adapted from [24]. The objective lens converts the incoming plane wavefront into a spherical one [38]. In terms of a modified Huygens-Fresnel principle, each point of the spherical wavefront in the exit pupil can be considered to give rise to a secondary plane wave [24, 107]. The intensity distribution in the focal region can then be described by a superposition of these secondary plane waves. To derive the phase masks for 3D tomoSTED, the intensity distribution was analysed along the directions in which an increase in resolution should be achieved. In three dimensions, all possible directions of resolution increase can be characterised by two angles. The angle $\psi$ was used to specify the rotation within the $\mathrm{x}-\mathrm{y}$ plane and the angle $\beta$ for the rotation from the $\mathrm{x}-\mathrm{y}$ plane towards the z-axis. 
Using polar coordinates, the electric field of the incoming wave for each position within the entrance pupil can be split into a part parallel to the radial direction and a part parallel to the direction of the polar angle $\phi\left(\vec{E}=\left(\vec{E} \overrightarrow{e_{r}}\right) \overrightarrow{e_{r}}+\left(\vec{E} \overrightarrow{e_{\phi}}\right) \overrightarrow{e_{\phi}}\right)$. Due to the operating mode of the objective lens, the part of the electric field parallel to $\overrightarrow{e_{r}}$ changes its direction by the angle $\theta$ and thus exhibits an additional component along the z-direction. The part parallel to the $\overrightarrow{e_{\phi}}$ remains unaffected: $\overrightarrow{e_{\phi}^{\prime}}=\overrightarrow{e_{\phi}}$ (see figure 5.1. In order to describe the direction of the electric field of the propagation wave for each point $(\mathrm{r}, \phi)$ of the exit pupil, a modified radial unit vector $\overrightarrow{e_{r}^{\prime}}$ can be defined (see equation (86) ) [24, 38, 73]. Additionally, the objective lens is assumed to obey the sine condition $(r=f \sin (\theta))$. Thus, the distance $r$ from the optical axis can be expressed via $\theta$. In the following, each point in the exit pupil is described via a $\theta$ - and a $\phi$-coordinate.

$$
\overrightarrow{e_{r}^{\prime}}=\left(\begin{array}{c}
\cos (\phi) \cos (\theta) \\
\sin (\phi) \cos (\theta) \\
\sin (\theta)
\end{array}\right)
$$

Furthermore, the energy has to be conserved along a ray passing through an optical system (energy conservation law) ([28],p.152) [24, 73]. This means that the energy of an ray passing through a surface segment at the entrance pupil $(d S=f \sin (\theta) d \phi d r)$ has to be equal to the energy of this ray at a surface segment of the exit pupil $\left(d S^{\prime}=f^{2} \sin (\theta) d \theta d \phi\right)$ :

$$
\left|\overrightarrow{E^{\prime}}\right|^{2} d S^{\prime}=|\vec{E}|^{2} d S
$$

From equation (87), it follows that the amplitude of the electric field at a position $(\theta, \phi)$ of the exit pupil equals the amplitude of the electric field at the entrance pupil position $(\theta, \phi)$ scaled by the factor $\sqrt{\cos (\theta)}$ :

$$
\left|\vec{E}^{\prime}\right|=|\vec{E}| \sqrt{\cos (\theta)}
$$

By means of equation (88), the electric field at the exit pupil can be determined [24]:

$$
\overrightarrow{E^{\prime}}(\theta, \phi)=\sqrt{\cos (\theta)}\left(\left(\vec{E} \overrightarrow{e_{r}}\right) \overrightarrow{e_{r}^{\prime}}+\left(\vec{E} \overrightarrow{e_{\phi}}\right) \overrightarrow{e_{\phi}^{\prime}}\right)
$$


The electric field in the exit pupil can thus be described as [38]

$$
\overrightarrow{E^{\prime}}(\theta, \phi)=\sqrt{\cos (\theta)} E_{0}\left(\begin{array}{c}
\cos \left(\phi-\phi_{0}\right) \cos (\theta) \cos (\phi)+\sin \left(\phi-\phi_{0}\right) \sin (\phi) \\
\cos \left(\phi-\phi_{0}\right) \cos (\theta) \sin (\phi)-\sin \left(\phi-\phi_{0}\right) \cos (\phi) \\
\cos \left(\phi-\phi_{0}\right) \sin (\theta)
\end{array}\right) P(\theta, \phi) .
$$

Within the Debye approximations, a plane wave can be defined for each point in the exit pupil which has a specific amplitude $\vec{E}^{\prime}(\theta, \phi)$ and a specific propagation direction ([28], p.147). The propagation direction is in the following expressed via the unit vector $\vec{s}$ pointing from a point in the aperture towards the focal point (origin) ([28, p.147). The electric field in a point within the focal region can then be determined by the summation of all components of the electric fields arriving in the observation point from all the points in the exit pupil ([28], p.147). For this, a vector $\vec{R}$ can be defined which points from the focal point (origin) to the observation point. The intensity is given by the square of the integration over the electric fields [24, 38, 173], ([28], p.147):

$$
I(\vec{R}) \propto\left|\int_{0}^{\alpha} \int_{0}^{2 \pi} \vec{E}^{\prime}(\theta, \phi) \mathrm{e}^{i k \vec{s} \vec{R}} \sin (\theta) \mathrm{d} \theta \mathrm{d} \phi\right|^{2}
$$

\subsubsection{Derivation of phase masks for the rotation in $y-z$ direction}

The idea of $3 \mathrm{D}$ tomoSTED is to rotate one dimensional depletion pattern in threedimensions which corresponds to a rotation of the high resolution axis. The direction of the high resolution axis can be specified by two angles. The angle $\psi$ was used within this thesis for the rotation within the $\mathrm{x}-\mathrm{y}$ plane and the angle $\beta$ for the rotation from the $\mathrm{x}-\mathrm{y}$ plane towards the $\mathrm{z}$-axis (see figure 5.1). Due to the radial symmetry of the optical system, it is sufficient to determine potential phase masks for the rotation of the high resolution axis in three dimensions, only for a single angle $\psi$. All other directions can then be obtained by rotating the phase masks in the $\mathrm{x}-\mathrm{y}$ plane by the angle $\psi$. Within this thesis, the $\mathrm{y}-\mathrm{z}$ direction was selected for this purpose.

Ideally, the derived phase masks should include the phase masks known from literature for an optimum resolution increase in $y$ - and $z$ direction. The optimum phase mask for a resolution increase along the $y$ direction is a $0-\pi$ phase step in $y$-direction [50, 51]. It has to be additionally combined with x-polarised light so that the intensity within the focal point equals zero [50, 51]. The phase mask for a optimum resolution enhancement in $z$-direction is the axial phase mask as shown in equation 10 in section 2.2. The axial 
phase mask works with both linearly polarised light and circularly polarised light [51]. Since the phase mask for the resolution increase in $\mathrm{y}$-direction requires $\mathrm{x}$-polarised light, $\mathrm{x}$-polarised light is used in the following to derive the phase mask for the rotation in the $y-z$ direction. The intensity distribution within in the focal region for x-polarised light can be obtained from equation (91) and is given by

$$
I(\vec{R}) \propto\left|\int_{0}^{\alpha} \int_{0}^{2 \pi} P(\theta, \phi) \sqrt{\cos (\theta)}\left(\begin{array}{c}
\cos (\theta)+(1-\cos (\theta)) \sin ^{2}(\phi) \\
(\cos (\theta)-1) \cos (\phi) \sin (\phi) \\
\cos (\phi) \sin (\theta)
\end{array}\right) \mathrm{e}^{i k \vec{s} \vec{R}} \sin (\theta) \mathrm{d} \theta \mathrm{d} \phi\right|^{2} .
$$

Subsequently, the intensity distribution is analysed along the desired axis of resolution increase which is rotated by an angle $\beta$ from the y-axis towards the z-axis. This rotated axis is named y' in the following (see figure 5.1). With this, the path lengths $(\vec{s} \vec{R})$ of the different plane waves arriving from different points in the exit pupil on the y' axis can be expressed by the rotation angle $\beta$ and $\mathrm{y}^{\prime}$ :

$$
\vec{s} \vec{R}=y^{\prime}(\sin (\beta) \cos (\theta)-\cos (\beta) \sin (\theta) \sin (\phi))=y^{\prime} d(\beta, \theta, \phi) .
$$

In equation (93), y' describes the distance from the focal point to the observation point along the y' axis. For further considerations, equation (93) is substituted into equation (92),

$$
I\left(\beta, y^{\prime}\right) \propto\left|\int_{0}^{\alpha} \int_{0}^{2 \pi} P(\theta, \phi) \vec{c}(\theta, \phi) \mathrm{e}^{i k y^{\prime} d(\beta, \theta, \phi)} \sin (\theta) \mathrm{d} \theta \mathrm{d} \phi\right|^{2},
$$

where the amplitude $\vec{c}(\theta, \phi)$ is given by

$$
\vec{c}(\theta, \phi)=\sqrt{\cos (\theta)}\left(\begin{array}{c}
\cos (\theta)+(1-\cos (\theta)) \sin ^{2}(\phi) \\
(\cos (\theta)-1) \cos (\phi) \sin (\phi) \\
\cos (\phi) \sin (\theta)
\end{array}\right)
$$

Any remaining intensity of the depletion beam at the focal point results in a reduction of the fluorescence intensity of the remaining effective PSF. Hence, a suitable phase mask for a resolution increase along a certain $\beta$ direction should lead to a depletion beam intensity of zero at the focal point. Thus, the zero intensity condition at the focal point has to be fulfilled for any $\beta$ direction:

$$
I(\beta, 0) \propto\left|\int_{0}^{\alpha} \int_{0}^{2 \pi} P(\theta, \phi) \vec{c}(\theta, \phi) \sin (\theta) \mathrm{d} \theta \mathrm{d} \phi\right|^{2} \stackrel{!}{=} 0 .
$$


The integration in equation $(96)$ depends on the amplitude $\vec{c}(\theta, \phi)$ and on the phase mask. Figure 5.2 therefore illustrates the contribution of the amplitude $\vec{c}(\theta, \phi)$ for different points of the exit pupil.
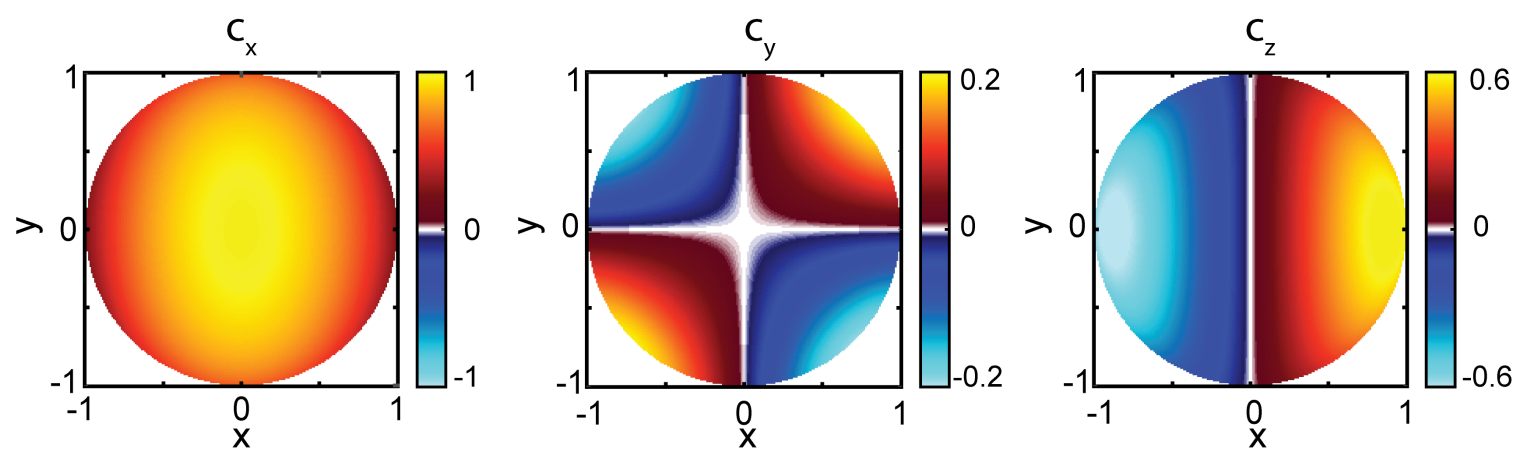

Figure 5.2: Illustration of the electric field's amplitude $\vec{c}(\theta, \phi)$ for the case of incoming $\mathrm{x}$ polarised light. In contrast to the $\mathrm{x}$-component of the amplitude, the $\mathrm{y}$-and the $\mathrm{z}$-component change their signs over the aperture. Therefore, the y-and the z-component will cancel each other out for in $\mathrm{x}$ mirror symmetric phase masks.

As expected due to the choice of $\mathrm{x}$-polarised light, the $\mathrm{x}$-component of the amplitude is significantly larger than the $\mathrm{y}$-component and the z-component. Moreover, the $\mathrm{x}$ component of the amplitude decreases towards the edge of the aperture, whereas the influence of the $\mathrm{y}$ - and $\mathrm{z}$-component increases. In contrast to the $\mathrm{x}$-component, the $\mathrm{y}$ and the $\mathrm{z}$-component change their signs across the aperture. More precisely, on the $\mathrm{x}$ axis opposite contributions exhibit the same amount but opposite signs. From this, it can be concluded that the contributions in the y-and z-components of the intensity will cancel each other out as long as a phase mask is applied that is mirror symmetrical in the $\mathrm{x}$ direction. Therefore, in the following the search is restricted to phase masks that are mirror symmetrical to $\mathrm{x}$. For such phase masks, it is sufficient to only consider the contribution of the x-component of the electric field to the intensity:

$$
I_{x}\left(\beta, y^{\prime}\right) \propto\left|\int_{0}^{\alpha} \int_{0}^{2 \pi} P(\theta, \phi) c_{x}(\theta, \phi) \mathrm{e}^{i k y^{\prime} d(\beta, \theta, \phi)} \sin (\theta) \mathrm{d} \theta \mathrm{d} \phi\right|^{2} .
$$

In addition to the zero intensity condition at the focal point, the phase mask should also lead to a depletion pattern which exhibits a steepness as high as possible along the y'-direction in proximity to the focal point. The reason for this is that with increasing steepness, the efficiency of the fluorescence suppression is enhanced (see equation (16) in section 2.2). The steepness is proportional to the second derivative of the intensity distribution along the y' direction. Therefore, the second derivative to y' from equation 
(97) has to be maximal at the focal point $y^{\prime}=0$, leading to

$$
\left.\frac{\partial^{2} I\left(\beta, y^{\prime}\right)}{\partial y^{\prime 2}}\right|_{y^{\prime}=0} \propto \cdot 2 k^{2}\left(\int_{0}^{\alpha} \int_{0}^{2 \pi} P(\theta, \phi) c_{x}(\theta, \phi) d(\beta, \theta, \phi) \sin (\theta) \mathrm{d} \theta \mathrm{d} \phi\right)^{2} \stackrel{!}{=} \max
$$

Equation (98) shows that the steepness is not only influenced by the contribution of the phase mask and the amplitude but also by the function $d(\beta, \theta, \phi)$. As illustrated in figure 5.2, $c_{x}(\theta, \phi)$ is approximately constant in the centre of the aperture and decreases mainly only near the edge. In order to simplify the equation $(98), c_{x}(\theta, \phi)$ is therefore first set to be constant. To fulfil the central zero intensity condition, the integration of the function $P(\theta, \phi)$ of the phase mask over the aperture has to become zero. The function $P(\theta, \phi)$ only possesses values of 1 or -1 . Therefore, the aperture has to be split into two parts of equal area. The phase of one half must be delayed by a phase of $\pi$ and the other half by a phase of 0 . In addition, to optimise the steepness, the integration of the multiplication of the function $P(\theta, \phi)$ by the factor $d(\beta, \theta, \phi)$ over the aperture has to be maximised. This condition is mainly influenced by the factor $d(\beta, \theta, \phi)$. Therefore, $d(\beta, \theta, \phi)$ is examined in the following and displayed in figure 5.3 .
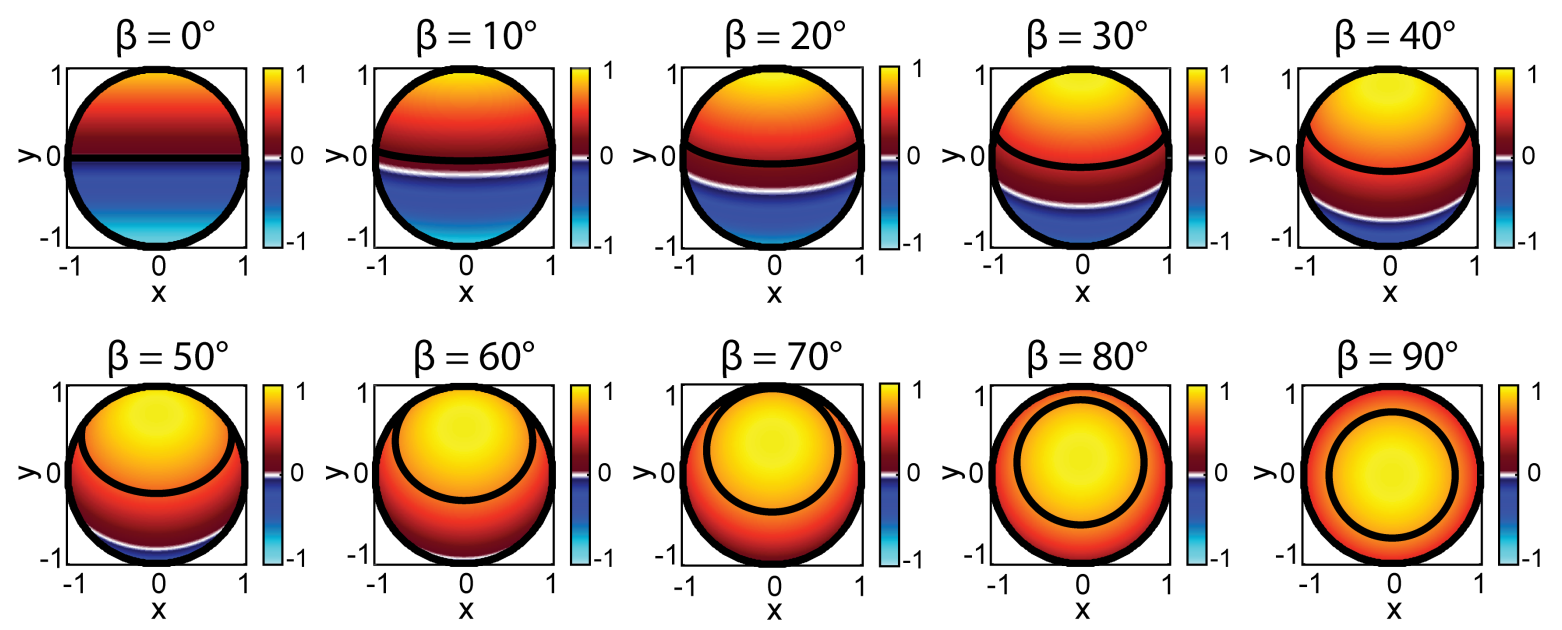

Figure 5.3: Illustration of the factor $d(\beta, \theta, \phi)$ as a function of angle $\beta$ including its median shown in black. By considering the median, the aperture is split into two halves leading to phase mask fulfilling both the zero intensity condition and the maximum steepness condition.

The black lines in figure 5.3 represent the median of $d(\beta, \theta, \phi)$. The median separates the values of $d(\beta, \theta, \phi)$ into two parts. One half with values greater than the median and the other half with values less than the median. Thus, by considering the median, the aperture is split into two halves. If the phase of one of these halves is chosen to be $\pi$ and a phase of 0 is applied to the other half, the intensity at the focal point equals 
zero. Thus, the first condition is fulfilled. To examine the steepness, the phase masks for low $\beta$-values are regarded firstly. For these masks, the median lies at zero or close to zero. This means the values of $d(\beta, \theta, \phi)$ separated by the median exhibit different signs. When choosing the phase mask so that the function $P(\theta, \phi)$ is 1 in one of the half and -1 in the other half, the signs become identical and only exhibit positive or negative contributions, leading to a maximum steepness (see equation (104)). Thus, the second condition of maximum steepness is also satisfied for these phase masks. At higher $\beta$ angles, the value of the median increases. Thus, $d(\beta, \theta, \phi)$ exhibits values with an identical sign in both halves. If the function $P(\theta, \phi)$ is chosen to possess a value of 1 in one half and -1 in the other half to satisfy the zero intensity condition, there will no longer be only positive or negative contributions. Therefore, the steepness will be lower for phase masks with higher $\beta$ angles. However, as shown in figure 5.3, the strengths of the values of $d(\beta, \theta, \phi)$ with an equal sign are different in the two halves. The values of $d(\beta, \theta, \phi)$ with an identical sign exhibit a lower strength in one half and are the values that contribute negatively to the steepness. However, as the strength of the negative contribution is smaller, the choice of the phase mask leads to the maximum possible steepness even at high $\beta$ angles if both conditions, the zero intensity condition and the maximum steepness condition, are to be fulfilled.

In summary, for an approximately constant amplitude, the phase masks found by the median satisfy within this simplification the condition of an intensity zero at the focal point and a steepness close to the highest possible. Furthermore, the steepness decreases from the case of $\beta=0^{\circ}$ towards $\beta=90^{\circ}$ since the value of the median increases. Thus, the highest possible $1 \mathrm{D}$ resolution is expected for $\beta=0^{\circ}$ and the lowest for $\beta=90^{\circ}$ while using the same STED laser power. Additionally, they are mirror symmetric in $\mathrm{x}$-direction and they include the 1D phase mask for the resolution increase in $\mathrm{y}$-direction $\left(\beta=0^{\circ}\right)$ and the axial phase mask $\left(\beta=90^{\circ}\right)$ known from literature. Thus, both the approach and the phase masks found seem to be suitable. The phase masks are only determined by the median which allows for a simple parametrisation. Therefore, they are well implementable in the experiment.

The error due to the simplification used within the approach by assuming a constant amplitude factor $c_{x}(\theta, \phi)$ mainly affects the depletion patterns for higher $\beta$ angles $\left(\beta=90^{\circ}\right)$ (see figure 5.2). The strength of the amplitude $c_{x}(\theta, \phi)$ decreases towards the edge of the aperture. Therefore, the phase mask determined by the median will not lead to a complete zero intensity at focal point in particular for higher $\beta$ angles $\left(\beta=90^{\circ}\right)$. However, 
this error can be easily corrected by using a suitable quantile (close to the median) of the distribution of $d(\beta, \theta, \phi)$ over the aperture instead.

\subsubsection{Validation of the phase masks for the rotation in $y-z$ direction on simulated data}

To validate the suitability of the theoretically derived phase mask, the performance of the phase masks was examined by simulations. ${ }^{16}$ In a first step, the intensity distribution of the depletion (STED) beam, generated by the phase masks, was examined. As discussed in the previous section 5.1.2, the median value of the factor $d(\beta, \theta, \phi)$ has to be slightly corrected in order to create completely zero intensity at the focal point. To find the optimal quantiles, STED beam intensity distributions with phase masks, generated with varying quantile values, were calculated ${ }^{17}$ A STED beam exhibiting zero intensity at the focal point leads to an effective PSF with maximum intensity. Thus, the quantile value for the phase mask was taken which leads to a maximum intensity of the effective $\mathrm{PSF} 18$ The maximum intensity as a function of the quantiles employed is shown in figure 5.4 as an example for $\beta=70^{\circ}$.

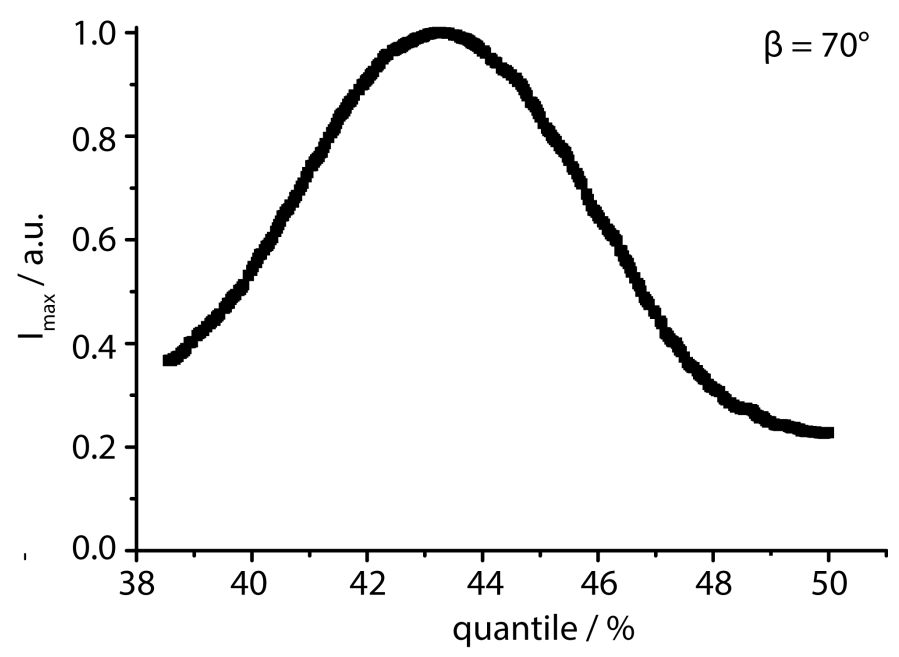

Figure 5.4: Maximum intensity of the effective PSF as a function of the quantile used for the $3 \mathrm{D}$ tomoSTED phase mask for $\beta=70^{\circ}$.

\footnotetext{
${ }^{16}$ The simulations were carried out using the 'Optical Microscopy Matlab Toolbox' of the Department for NanoBioPhotonics, of the Max Planck Institute for biophysical Chemistry in Göttingen. The 'Optical Microscopy Matlab Toolbox' calculates the intensity distribution according to equation (91). The effective PSF is determined via equation (11).

${ }^{17}$ The simulations were carried out using a STED beam wavelength of $\lambda_{\mathrm{STED}}=775 \mathrm{~nm}$ and a oil immersion objective $\left(n_{\text {oil }}=1.515\right)$ featuring a numerical aperture of 1.4.

${ }^{18} \mathrm{Here}$, the effective PSFs were calculated using an excitation wavelength of $640 \mathrm{~nm}$, an emission wavelength of $680 \mathrm{~nm}$ and a saturation factor of 120 .
} 
As illustrated in figure 5.4, the maximum intensity of the effective PSF and therefore zero intensity of the STED beam at the focal point is strongly influenced by the quantile value used for the phase mask. For $\beta=70^{\circ}$, employing a phase mask determined by the median and a saturation factor of 120 resulted in an intensity loss of $80 \%$. The phase masks leading to the maximum intensity of the effective PSF are shown in figure 5.5 for different values of $\beta$ together with $y$-z cross sections of the STED beam intensity distribution generated by these phase masks.
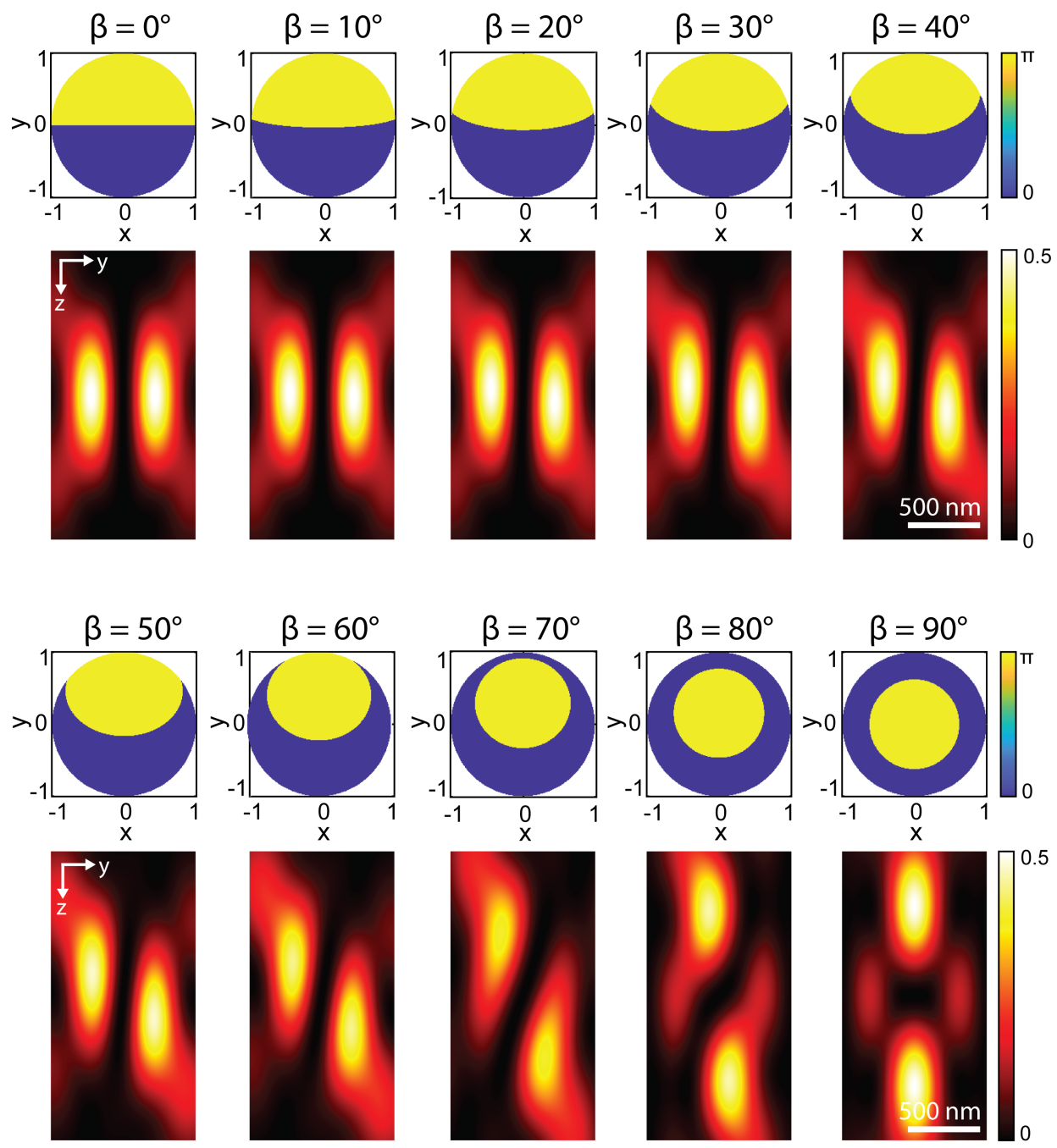

Figure 5.5: Phase masks depending on the rotation angle $\beta$ created with a corrected quantile value as well as $\mathrm{y}-\mathrm{z}$ cross sections of the resulting STED intensity distribution for simulated data. The following quantiles were used to simulated the STED intensity distributions: $50 \%$ $\left(\beta=0^{\circ}\right), 49.1 \%\left(\beta=10^{\circ}\right), 48.8 \%\left(\beta=20^{\circ}\right), 47.8 \%\left(\beta=30^{\circ}\right), 47.6 \%\left(\beta=40^{\circ}\right), 46.8 \%$ $\left(\beta=50^{\circ}, 45.5 \%\left(\beta=60^{\circ}\right), 43.6 \%\left(\beta=70^{\circ}\right), 43.4 \%\left(\beta=80^{\circ}\right), 43.3 \%\left(\beta=90^{\circ}\right)\right.$. All STED beam intensity distributions exhibit a clear zero intensity within the focal point and show a rotation of the maximum intensity in relation to the employed $\beta$-angle. 
All STED intensity distribution exhibit zero intensity at the focal point. Depending on the $\beta$-angle used for creating the phase masks, the line between peaks of the STED intensity distribution rotates in the $\mathrm{y}$-z plane. An intensity line leading through the maxima typically coincides with the highest curvature direction. Thus, ideally the angle by which the line trough the maxima is rotated corresponds to the $\beta$-angle used for generating the phase mask. To examine the position of the maxima in relation to $\beta$, the angles of the maxima with respect to the y-axis were determined. Afterwards, the two angles obtained for the two maxima of each intensity distribution were averaged. The results are presented in figure 5.6.

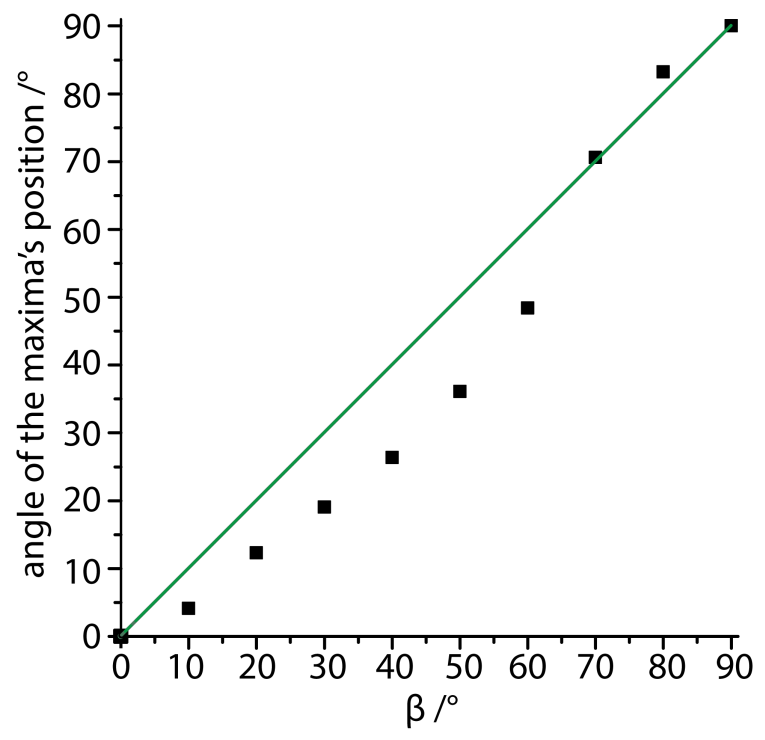

Figure 5.6: Angle of the maxima's position in relation to $\beta$ together with a bisecting line drawn in green.

The green line in figure 5.6 illustrates the bisecting line on which the determined angle of the maxima should ideally lie on. The determined angles of the maxima are close to the expected line. However, especially for the angles in between $\left(40^{\circ}\right.$ and $\left.50^{\circ}\right)$, they deviate clearly. An explanation might be that the tilting capability is somehow limited by the aperture angle of the objective lens. However, the maxima rotate continuously and therefore it is possible to adjust any angle, even if it deviates slightly from $\beta$.

The applicability of the phase masks was additionally investigated experimentally by measuring the STED intensity distribution using a gold bead sample ${ }^{19}$. The STED in-

\footnotetext{
${ }^{19}$ The gold bead sample consisted of 150 nm-sized gold beads (BBI Solutions, United Kingdom) which were immobilised on a cover slide surface coated with Poly-L-lysine $(0.1 \%(\mathrm{w} / \mathrm{v})$ in H2O, SigmaAldrich, USA), and were embedded in mowiol. The reflected light from the gold beads was recorded by a photomultiplier tube.
} 
tensity distributions measured for $\beta$ values of $0^{\circ}, 60^{\circ}, 70^{\circ}, 80^{\circ}$ and $90^{\circ}$ are presented in figure 5.7 .
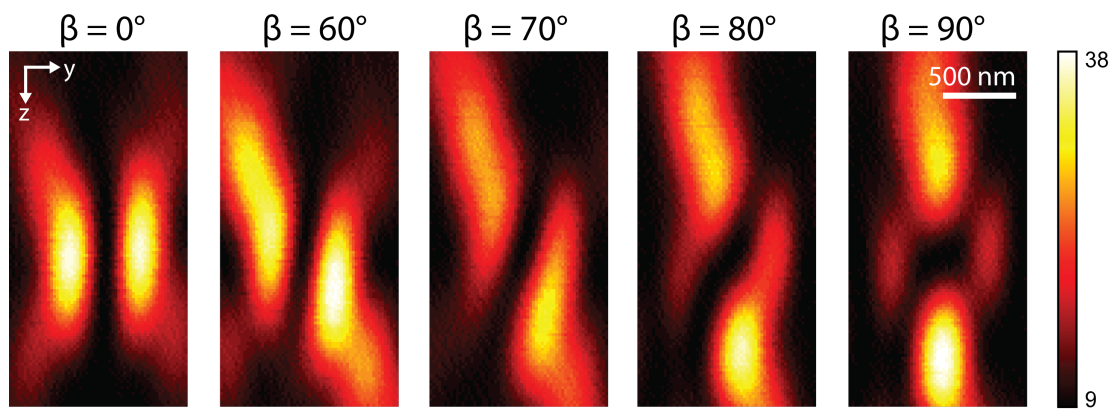

Figure 5.7: STED intensity distributions experimentally measured on $150 \mathrm{~nm}$-sized gold beads using the 3D tomoSTED phase masks as a function of $\beta$.

The experimentally measured depletion pattern exhibit a similar shape to the simulated data and clearly show zero intensity at the focal point. Furthermore, a rotation of the maxima depending on the beta angle can be observed. The phase masks therefore also seem to be applicable in the experiment.

The phase masks found can only be used effectively in 3D tomoSTED if they lead to an additional gain of high resolution information. To validate this, the effective PSFs were calculated as previously described. Cross sections along the y-z-axis of the resulting effective PSFs for different values of $\beta$ are shown in figure 5.8 (a).

As shown in figure 5.8, the high-resolution axis of the effective PSFs rotates as a function of $\beta$. However, the use of the phase masks is only advantageous and thus reasonable if they achieve a higher resolution at least in a certain direction in the $\mathrm{y}-\mathrm{z}$ plane than the $\beta=0^{\circ}$ - and $\beta=90^{\circ}$-PSF already known from the literature. Therefore, the width of the effective PSFs was examined in different directions rotated by the angle $\theta$ with respect to the y-axis. More precisely, line profiles were drawn through each effective $\mathrm{PSF}$ and these were additionally rotated by the angle $\theta$. The value of $\theta$ was varied in $1^{\circ}$ steps between $0^{\circ}$ and $90^{\circ}$. For each $\theta$ angle, the FWHM of the intensity line profile was determined by a Gaussian fit. The widths obtained as a function of the $\theta$ angles for different $\beta$ angles are shown in figure 5.8 (b). It is shown that effective PSFs exist which exhibit a significantly smaller width within a certain angular range $\theta$ than the $0^{\circ}$ and $90^{\circ}$ PSF known from the literature. The phase masks found can thus be used to achieve an additional gain in high-resolution information and are therefore suitable for the application in 3D tomoSTED. 
(a)
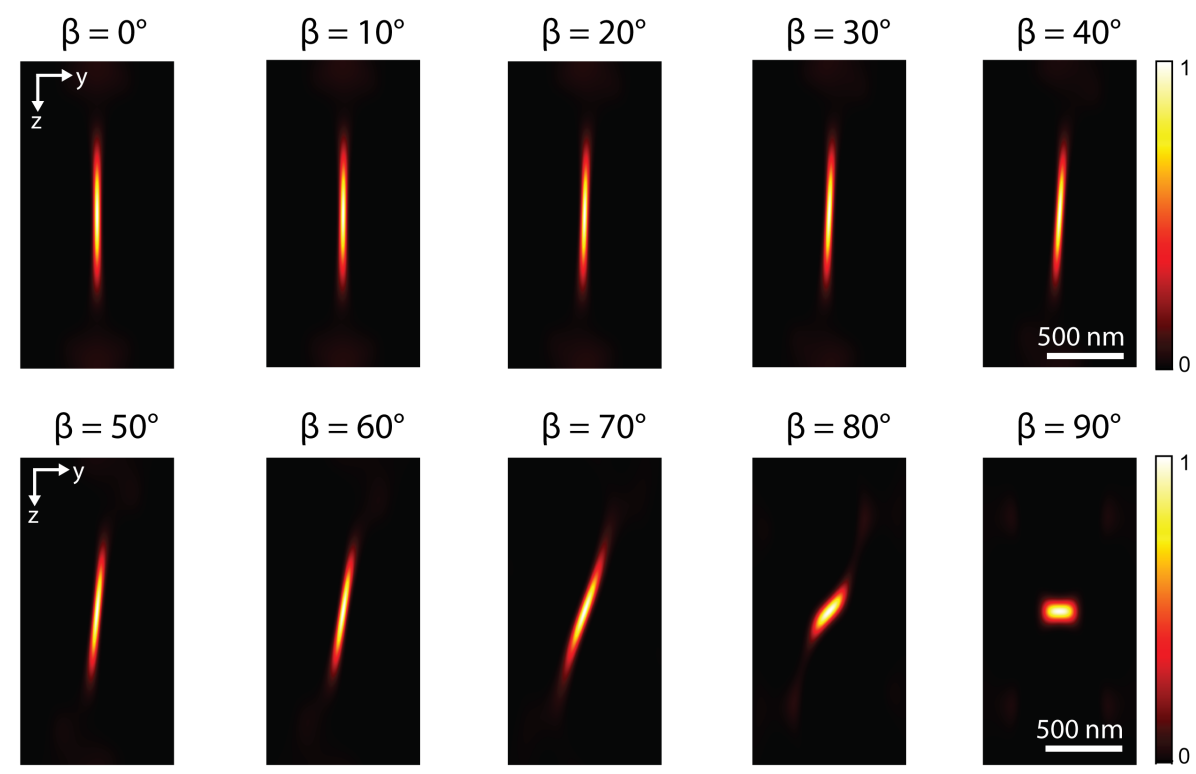

(b)
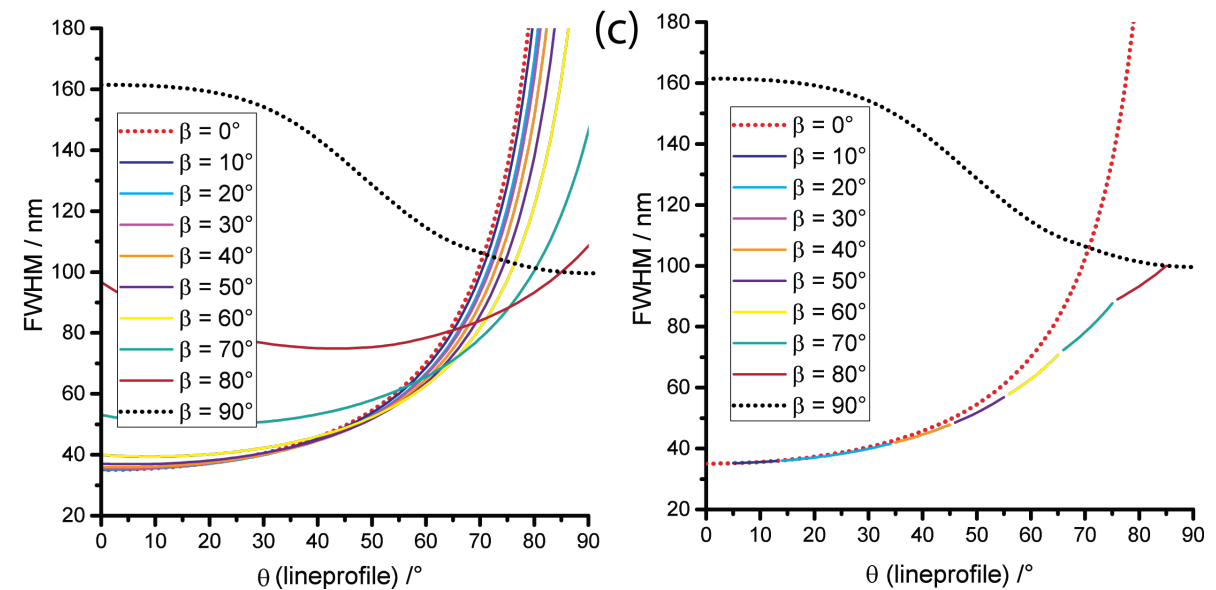

Figure 5.8: Effective PSFs resulting from the 3D tomoSTED phase masks. (a) Y-z crosssection of effective PSFs for different values of $\beta$. (b) Width of the effective PSFs for different values of $\beta$ in the direction of $\theta$. (c) shows the same curves as in (b) for $\beta=0^{\circ}$ and for $\beta=90^{\circ}$. For all other values of $\beta$, only the curve section, which exhibits the smallest widths of all curves, is displayed.

To further investigate this gain in additional high-resolution information, figure 5.8 (c) shows the same curve of width as a function of $\theta$ for the $0^{\circ}$ and $90^{\circ}$ PSFs. For all other $\beta$ angles, however, only the minimum width obtained from all effective PSFs under a certain line profile direction $\theta$ is displayed in figure 5.8 (c). A considerably smaller width than the $0^{\circ}$ or the $90^{\circ} \mathrm{PSF}$ could only be achieved for $\beta$-angles larger than $40^{\circ}$. Therefore, only orientations with $\beta$ angles greater than $40^{\circ}$ were selected for 3D tomoSTED, in the following. 


\subsection{Reduction factor for the laser power}

The aim of developing 3D tomoSTED is to obtain a three-dimensional imaging technique that achieves a lower light dose than the conventional 3D-STED while maintaining the same image quality. Therefore, the potential of each individual 3D tomoSTED PSF is investigated in the following with regard to a measurement at a reduced STED laser power. In order to obtain the same image quality as 3D-STED, at least an identical resolution has to be achieved. Thus, each single 3D tomoSTED PSF is examined for the maximum factor by which the laser power can be reduced. The maximum factor is reached when the direction of the highest resolution of the individual 3D tomoSTED PSF corresponds to the resolution of the conventional 3D-STED.

The maximum reduction factor for the laser power for each individual 3D tomoSTED PSF was estimated similarly as presented for 2D tomoSTED in section 2.3. Analogous to 2D tomoSTED (see equation (18)), also for 3D tomoSTED a factor $r^{2}$ can be defined which consists of the square of the FWHM ratio of the individual 1D tomoSTED PSF to that of the conventional 3D-STED:

$$
r^{2}=\left(\frac{\text { FWHM }_{\text {STED, 1D }}}{\text { FWHM }_{\text {STED, 3D }}}\right)^{2} .
$$

The factor $r^{2}$ represents the factor by which the laser power applied to each 3D tomoSTED orientation can be reduced relative to the laser power used for 3D STED to obtain the same FWHM along a direction of interest ${ }^{20}$.

$$
\zeta_{1 \mathrm{D}}=\frac{1}{r^{2}\left(\zeta_{1 \mathrm{D}}=\zeta_{3 \mathrm{D}}\right)} \zeta_{3 \mathrm{D}}
$$

To determine $r^{2}$, effective PSFs were simulated as described in section 5.1.3. In order to find the maximum $r^{2}$ for each individual 3D tomoSTED PSF ${ }^{21}$, the widths of the effective PSFs was examined in different $\theta$ direction similar to section 5.1.3. Thus, line profiles were drawn through the individual PSFs and these were additionally rotated by the angle $\theta$. The value of $\theta$ was varied from $0^{\circ}$ to $180^{\circ}$ in $1^{\circ}$ steps. To determine the width of each line profile, a Gaussian fit was performed. Afterwards, $r^{2}$ was determined for each $\theta$ as well as for each individual 3D tomoSTED PSF. The maximum value of $r^{2}$

\footnotetext{
${ }^{20}$ Please refer to section 2.3 for a more detailed description.

${ }^{21}$ Here, only the rotation along $\beta$ is considered. Thus, the orientations were created for a rotation along the y-z-direction. However, due to the radial symmetry, the results should also apply to all other rotations in $3 \mathrm{D}$.
} 
found for each individual 3D tomoSTED PSF from all $\theta$ directions investigated is shown in figure 5.9 as a function of the 3D tomoSTED orientation angle $\beta$.

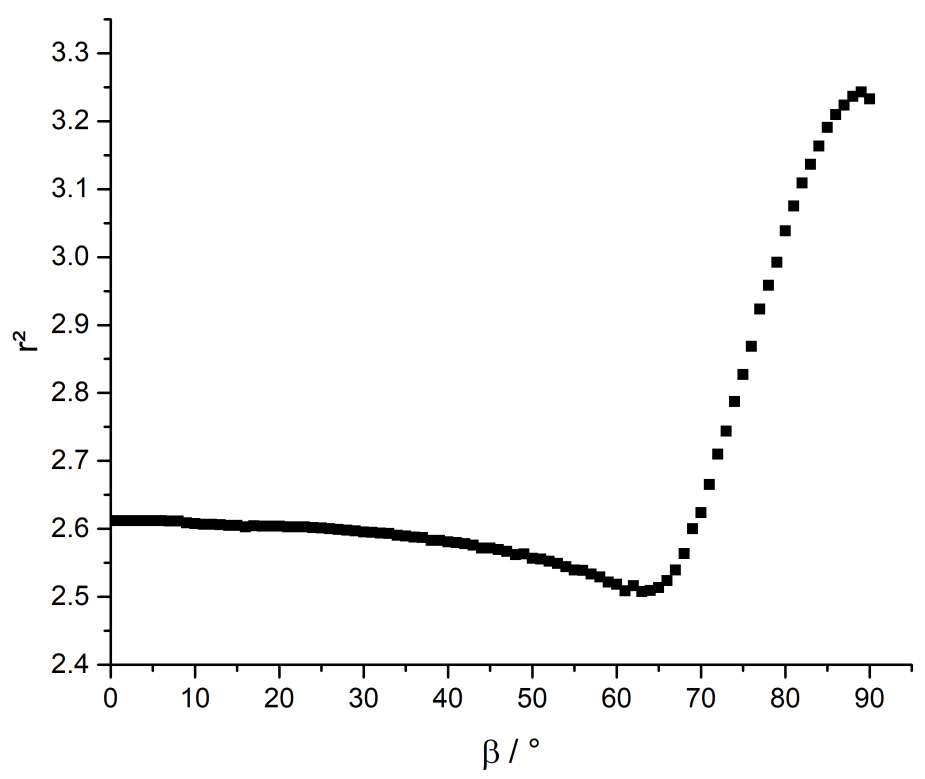

Figure 5.9: Maximum reduction factor for the laser power for the individual 3D tomoSTED PSFs as a function of $\beta$

As illustrated in figure 5.9, the maximum laser power reduction factor for the individual orientations ranges from 2.51 to 3.24. A common reduction factor for the laser power for all individual 3D tomoSTED orientations can be implemented more easily in the experiment. Therefore, in the following, a common reduction factor of 2.61 was selected, since a 2.61-fold lower laser power used for individual orientations achieves at least the same or a higher resolution in the lateral and axial directions than the conventional variant.

\subsection{Reduction factor for the exposure time}

The individual 3D tomoSTED PSFs are mainly reduced in size along one spatial direction. Therefore, the signal of the individual 3D tomoSTED PSFs is expected to be higher than the signal of the conventional 3D STED when considering the same resolution enhancement in the respective direction. Thus, the exposure time might be lowered compared to the conventional 3D STED in order to achieve the same signal strength. If the exposure time can be reduced, a lower light dose could be achieved. Therefore, the potential for lowering the exposure time is investigated in the following by comparing the signal of the individual 3D tomoSTED PSFs with that of the 3D STED. 
To estimate a reduction factor of the exposure time, the total signal of the individual 3D tomoSTED PSFs was compared to the one of the conventional 3D-STED. For this purpose, the STED laser power of the individual 3D tomoSTED PSFs was reduced by the factor of 2.61, whereas an unscaled laser power (a saturation factor of 120) was applied for the conventional variant. In addition, circularly polarised light was used to simulate the $\beta=90^{\circ} 3 \mathrm{D}$ tomoSTED PSF. The $\beta=90^{\circ}$ phase mask works with both circularly and linearly polarised light (see section 5.1.2). However, the use of circularly polarised light has the advantage that the same resolution is achieved along the z-axis as with linearly polarised light, but in contrast to linear polarisation, the width in the $\mathrm{x}$ - and $\mathrm{y}$-directions is the same. Thus, when using circularly polarised light, an isotropic resolution is achieved with respect to the $\mathrm{x}-\mathrm{y}$ plane, which is advantageous for the subsequent arrangement of 3D tomoSTED orientations.

The effective PSFs do not only show signal in the main peak, but also exhibit not negligible background signal. Examples of the background signal for the conventional 3D-STED as well as for the $\beta=0^{\circ}$ and $\beta=90^{\circ}$ orientation are displayed in figure 5.10 (a). Here, figure 5.10 (a) only shows the $y-z$ plane of the effective PSFs.
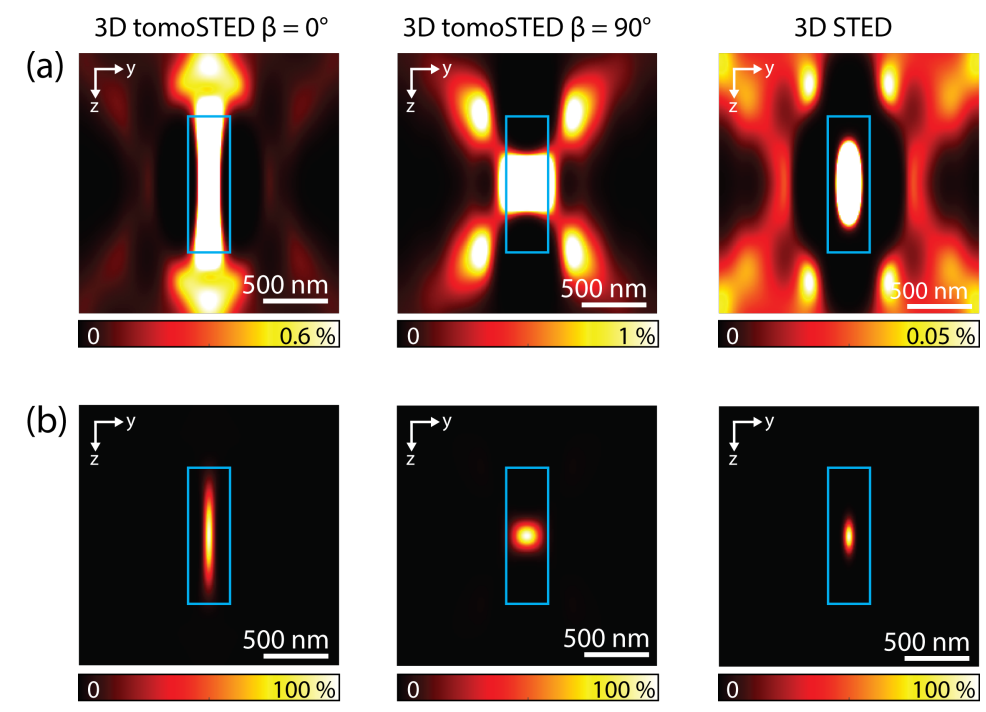

Figure 5.10: Illustration of (a) the effective PSFs' background signal for 3D-STED and the $\beta=0^{\circ}$ - and $\beta=90^{\circ} 3 \mathrm{D}$ tomoSTED orientation within the $\mathrm{y}-\mathrm{z}$ plane and $(\mathrm{b})$ of the cuboid used to determine the total intensity.

As illustrated in figure 5.10 (a), the background signal for 3D-STED is approximately a factor of 12 lower than that of the $\beta=0^{\circ}$ orientation and by a factor of 20 smaller compared to the $\beta=90^{\circ}$ orientation. Therefore, the whole field of view was not used to 
determine the total intensity, but only the intensity values within a cuboid were taken into account. As a compromise between the different background signals of the single PSFs, the lengths of the cuboid were chosen to be $1.05 \mu \mathrm{m}$ in z-direction, $470 \mathrm{~nm}$ in $\mathrm{x}$ direction and $310 \mathrm{~nm}$ in y-direction. These lengths correspond to the PSF width of the $\beta=0^{\circ}$ orientation in z-direction and in $\mathrm{x}$-direction and of the $\beta=90^{\circ}$ orientation in $\mathrm{y}$-direction at an intensity value of $5 \%$. For the $\mathrm{y}$-z plane, a cross section of the cuboid used together with the 3D-STED, the $\beta=0^{\circ}$ and the $\beta=90^{\circ}$ effective PSFs are shown in figure 5.10 (b). The used cuboid is there indicated by the blue rectangle.

To estimate the total signal of the PSFs, the intensity values within the cuboid were summed up and the ratio of these values compared to the conventional variant was determined. The result of these ratios as a function of the $\beta$-angle is shown in figure 5.11.

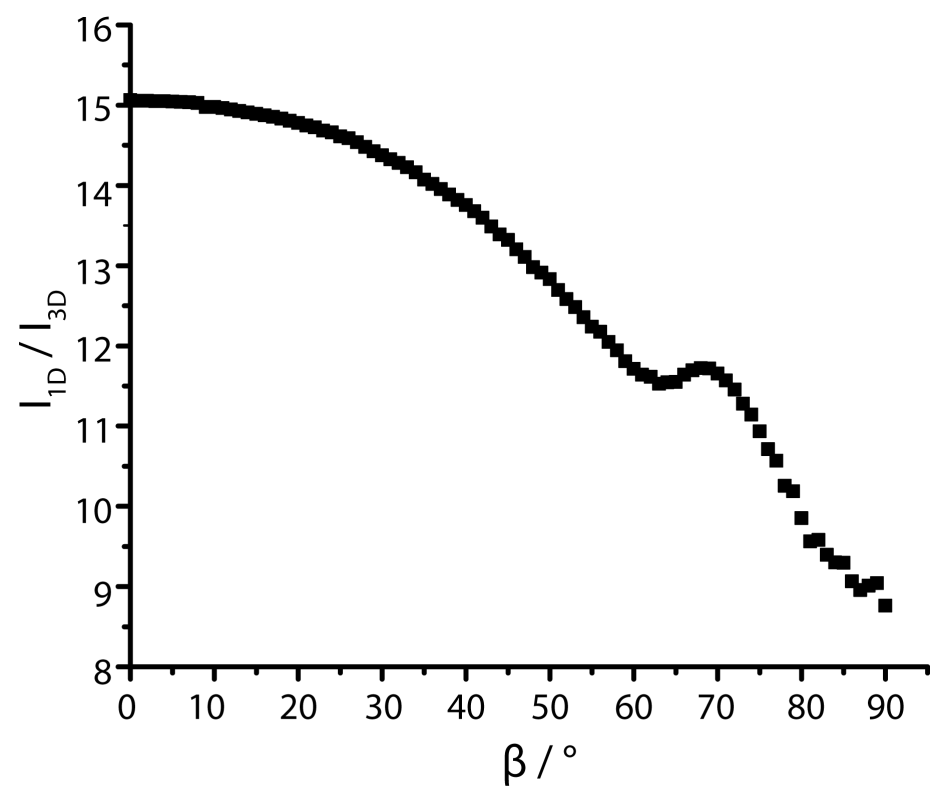

Figure 5.11: Total intensity of the individual 3D tomoSTED PSFs summed over the cuboid, calculated for a STED intensity reduced by a factor of 2.61, compared to the signal of the conventional 3D STED

As illustrated in figure 5.11, the ratio of the individual 3D tomoSTED PSFs to the signal of the conventional variant ranges from a factor of 15.1 to a factor of 8.8. To validate the suitability of these values, the values of the $\beta=0^{\circ}$ - and the $\beta=90^{\circ}$ PSF were compared to their theoretically expected values. Since the effective PSFs of these orientations can be approximated by a Gaussian [33, 59], the total intensity can be obtained 
by an integration over the volume of the corresponding Gaussian:

$$
\begin{aligned}
\iiint_{-\infty}^{+\infty} e^{-\frac{1}{2}\left(\frac{x^{2}}{\sigma_{x}^{2}}+\frac{y^{2}}{\sigma_{y}^{2}}+\frac{z^{2}}{\sigma_{z}^{2}}\right)} d x d y d z & =\sqrt{2 \pi}^{3} \sigma_{x} \sigma_{y} \sigma_{z} \\
& =\frac{\sqrt{2 \pi}^{3}}{(2 \sqrt{2 \ln 2})^{3}} \cdot \mathrm{FWHM}_{x} \mathrm{FWHM}_{y} \mathrm{FWHM}_{z}
\end{aligned}
$$

According to equation (101), the total intensity of a Gaussian depends on its respective widths. Thus, the signal ratio between two Gaussians can be obtained by comparing their widths. By comparing the PSF FWHMs of the $\beta=0^{\circ}$ - and the $\beta=90^{\circ}$ orientation to those of the conventional variant, a signal ratio of approximately 14.1 for the $\beta=0^{\circ}$ orientation and a ratio of about 8.3 for the $\beta=90^{\circ}$ orientation was obtained. This amounts to a relative deviation between the values, determined via simulations and the values, calculated via the widths, of $7 \%$ for the $\beta=0^{\circ}$ - and of $6 \%$ for the $\beta=90^{\circ}$ PSF. Reasons for the higher values might be the deviations from a single Gaussian peak shape. However, the determined values are close to the expected values. Hence, the signal determining procedure seems to be suitable for an estimation of the signal ratio of the individual orientations compared to the conventional 3D STED.

In order to be able to implement the reduction of the exposure time more easily in a later experimental implementation, a common reduction factor was chosen for all individual orientations in the following. In order to achieve at least the same signal strength in all orientations used as in the conventional 3D STED, the exposure time of the individual orientations can be reduced by the factor of the smallest signal ratio. Furthermore, it can be concluded that it is unfavourable in terms of the light dose if the number of orientations used is greater than the reduction factor of the exposure time, because then 3D tomoSTED shows a higher total exposure time than the conventional STED.

\subsection{Selection of 3D tomoSTED orientations}

As a continuous rotation is unfavorable with respect to the light dose (see section 5.3), a finite number of orientations has to be used for a 3D tomoSTED implementation. The respective chosen individual $3 \mathrm{D}$ tomoSTED PSFs should cover the spatial frequencies present in the conventional 3D STED PSF well enough so that the reconstructed final image exhibits at least the same resolution in all spatial directions compared to the conventional variant. In addition, the number of orientations should be as low as possible 
to achieve the greatest advantage in terms of light dose (see section 5.3).

So far, mainly individual 3D tomoSTED PSFs have been investigated with their highresolution direction rotated from the y-axis to the z-axis. However, to achieve high resolution in all spatial directions in three dimensions, the high-resolution direction also has to be rotated in the $\mathrm{x}-\mathrm{y}$ plane. The spatial orientation of each individual 3D tomoSTEDPSF can therefore be characterised by two angles, one describing the rotation of its high-resolution direction in the $\mathrm{x}-\mathrm{y}$ plane and the other describing its rotation from the $\mathrm{x}-\mathrm{y}$ plane towards the z-axis, as illustrated in figure 5.12 . Within this thesis, the orientation of the individual 3D tomoSTED PSFs was specified by the lateral angle $\psi$ and the axial angle $\beta$. These angles correspond to the angle used for the phase masks to generate individual 3D tomoSTED PSFs, exhibiting differently oriented high-resolution directions (see section 5.1). As shown in section5.1.3, the actual direction of the high-resolution for the $\beta$ direction differs slightly from the $\beta$ angle used for the phase mask.

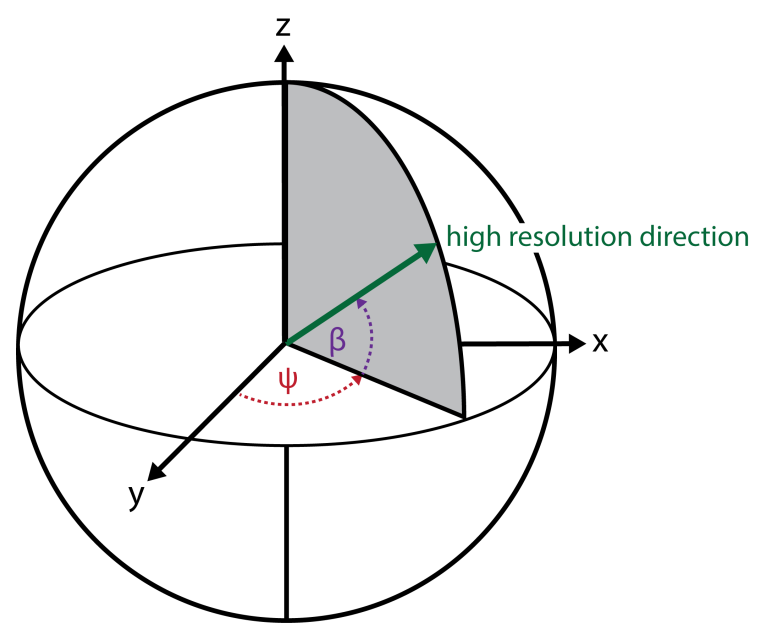

Figure 5.12: The orientation of each individual 3D tomoSTED PSF can be specified by two angles. Within this thesis, the orientation of the individual 3D tomoSTED PSFs was defined by the angles employed to generate them using the phase masks. The rotation within the $\mathrm{x}-\mathrm{y}$ plane was characterised by the angle $\psi$ and the rotation to the z-axis by the angle $\beta$. Along the $\beta$ direction, the actual direction of the high-resolution of the individual 3D tomoSTED PSFs differs slightly from the $\beta$ angle used for the phase masks, as shown in section 5.1.3.

Many possibilities exist for arranging the individual 3D tomoSTED PSFs in threedimensional space. In order to obtain a first impression of the performance of the 3D tomoSTED, three different arrangements were investigated: one with a low number of orientations, one with a medium number and one with a high number. All arrangement included individual 3D tomoSTED PSFs generated with $\beta=0^{\circ}$ and $\beta=90^{\circ}$ as they lead to an optimal resolution enhancement in the $\mathrm{x}-\mathrm{y}$ plane and along the $\mathrm{z}$-direction. 
The arrangement of 9 orientations was investigated for the case of a small number of orientations. For this arrangement, individual PSFs with $\beta$ angles of $0^{\circ}, 90^{\circ}$ and one more $\beta$ angle $\left(\beta=67^{\circ}\right)$ were selected. For the rotation in the lateral plane, four $\psi$ angles were used, respectively for $\beta=0^{\circ}$ and $\beta=67^{\circ}$. 19 number of orientations were examined for an arrangement with a medium number of orientations. For this arrangement, four $\beta$ angles $\left(0^{\circ}, 62^{\circ}, 67^{\circ}\right.$ and $\left.90^{\circ}\right)$ and six lateral $\psi$ angles were selected. The arrangement with a high number of orientations consisted of 153 orientations. Here, individual PSFs were chosen with $\beta$-angles increasing from $45^{\circ}$ to $90^{\circ}$ in $5^{\circ}$ steps in addition to $\beta=0^{\circ}$. Moreover, eight lateral $\psi$ angles were selected for $\beta=0^{\circ}$ and 16 for the other $\beta$ angles except for $\beta=90^{\circ}$. In the following, the selection of the two $\beta$-angles $\left(\beta_{1}\right.$ and $\left.\beta_{2}\right)$ in addition to $\beta=0^{\circ}$ and $\beta=90^{\circ}$ for the case of the 19 orientations is described in more detail. The additional $\beta$-angle for the arrangement of the 9 orientations was chosen in a similar way.

To achieve the highest isotropic coverage of spatial frequencies, the 6 lateral rotation angle $\psi$ of the individual 3D tomoSTED PSFs with $\beta=0^{\circ}$ were chosen to be equally spaced within the $\mathrm{x}-\mathrm{y}$ plane. Thus, the values of $\psi$ were selected to be $0^{\circ}, 30^{\circ}, 60^{\circ}, 90^{\circ}$, $120^{\circ}$, and $150^{\circ}$. In order to cover the three-dimensional space as well as possible, the lateral rotation angles of the individual PSFs with angles of $\beta_{1}$ and $\beta_{2}$ were set such that each individual $\beta_{1}$-PSF lies in between two individual $\beta_{2}$-PSFs. Furthermore, all these individual PSFs were rotated laterally into the spaces between the individual $\beta=0^{\circ}$ PSFs. More precisely, $\psi$ angles of $15^{\circ}, 75^{\circ}, 135^{\circ}, 195^{\circ}, 255^{\circ}$, and $315^{\circ}$ were employed for the $\beta_{1}$-individual PSFs. For the $\beta_{2}$-individual PSFs, $\psi$-angles of $45^{\circ}, 105^{\circ}, 165^{\circ}, 225^{\circ}$, $285^{\circ}$, and $345^{\circ}$ were used. ${ }^{22}$

To identify the most appropriate values for $\beta_{1}$ and $\beta_{2}$, the resolving capability of the final 3D tomoSTED images reconstructed from different combinations of $\beta_{1}$ and $\beta_{2}$ were examined. The maximum-value reconstruction represents a direct way to investigate the resolution in terms of spatial frequencies present in the final reconstructed images (see section 3.3.1). Therefore, the maximum-value reconstruction was chosen here as reconstruction method. As described in section 3.3.1, the maximum-value reconstruction represents a linear reconstruction method [58]. This means that the final image

\footnotetext{
${ }^{22}$ For the $\beta=0^{\circ}$-individual PSFs, PSFs rotated by $180^{\circ}$ are identical. Thus, the lateral rotation of these orientations can be chosen according to $180^{\circ} / N$ due to the equal spacing. Here, $\mathrm{N}$ describes the number of lateral orientations. For individual PSFs with higher $\beta$-angles except for the $\beta=90^{\circ}$, two orientations correspond to each other that are rotated by $360^{\circ}$. Thus, a lateral rotation until $360^{\circ}$ has to be considered for all individual PSFs with $\beta$-angles not equal to $0^{\circ}$ and $90^{\circ}$.
} 
can be described as a convolution of the object underlying the image with an effective PSF. This effective PSF is given by the maximum-value reconstruction of the individual 3DtomoSTED PSFs. Thus, to quantify the resolving capability, the width of the effective PSFs obtained with different combinations of $\beta_{1}$ and $\beta_{2}$ angles were examined. For this, line profiles averaged over three neighbouring pixels were drawn in $\mathrm{x}-, \mathrm{y}$ - and z-direction through the effective PSFs and the FWHMs were determined. For the example of 19 orientations, the obtained FWHMs are shown in figure 5.13 (a).

(a)

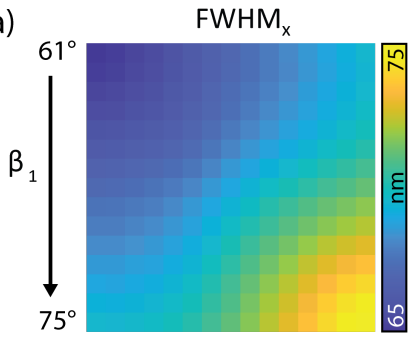

(b)

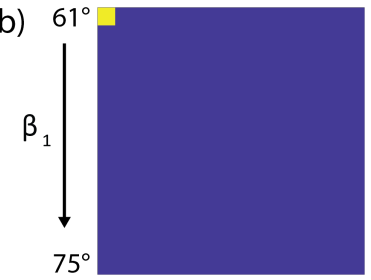

(c)

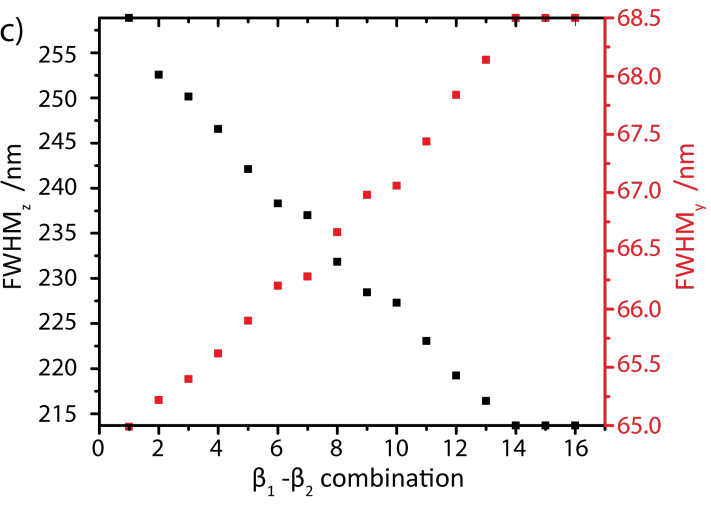

FWHM $_{\mathrm{y}}$
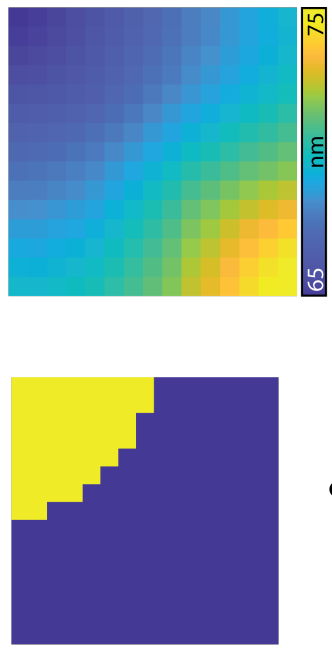

$\mathrm{FWHM}_{2}$

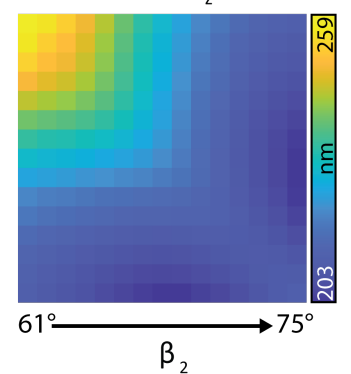

$\bullet$

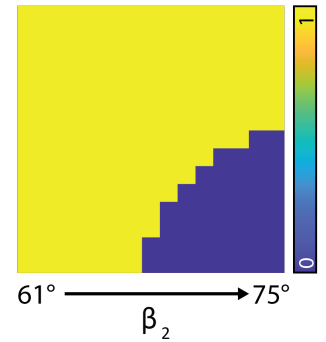

$\beta_{1}-\beta_{2}$ combination:

1: $\beta_{1}=61^{\circ}, \beta_{2}=61^{\circ} \quad 9: \beta_{1}=61^{\circ}, \beta_{2}=68^{\circ}$ $2: \beta_{1}=62^{\circ}, \beta_{2}=62^{\circ} \quad 10: \beta_{1}=62^{\circ}, \beta_{2}=68^{\circ}$ 3: $\beta_{1}=62^{\circ}, \beta_{2}=63^{\circ} \quad 11: \beta_{1}=62^{\circ}, \beta_{2}=69^{\circ}$ 4: $\beta_{1}=62^{\circ}, \beta_{2}=64^{\circ} \quad 12: \beta_{1}=62^{\circ}, \beta_{2}=70^{\circ}$ 5: $\beta_{1}=62^{\circ}, \beta_{2}=65^{\circ} \quad 13: \beta_{1}=62^{\circ}, \beta_{2}=71^{\circ}$ $6: \beta_{1}=61^{\circ}, \beta_{2}=66^{\circ} \quad 14: \beta_{1}=62^{\circ}, \beta_{2}=72^{\circ}$ 7: $\beta_{1}=62^{\circ}, \beta_{2}=66^{\circ} \quad 15: \beta_{1}=62^{\circ}, \beta_{2}=72^{\circ}$ 8: $\beta_{1}=62^{\circ}, \beta_{2}=67^{\circ} \quad 16: \beta_{1}=62^{\circ}, \beta_{2}=72^{\circ}$

Figure 5.13: Illustration of the selection of the angles $\beta_{1}$ and $\beta_{2}$ for the arrangement of 3D tomoSTED with 19 orientations. (a) FWHMs in $\mathrm{x}-, \mathrm{y}-$ and $\mathrm{z}$-direction of effective PSFs reconstructed for different combinations of values for the angles $\beta_{1}$ and $\beta_{2}$ (from $61^{\circ}$ to $75^{\circ}$ ) using the maximum value reconstruction. (b) Illustration of the procedure for selecting the $\beta_{1^{-}}$ $\beta_{2}$ combination. The effective PSFs of $\beta_{1}-\beta_{2}$ combinations whose FWHM in y-direction exhibits a value below a certain threshold were investigated. The $\beta_{1^{-}} \beta_{2}$ combinations considered accordingly are marked in yellow. From these $\beta_{1}-\beta_{2}$ combinations, the combination was selected whose effective PSF exhibits the smallest volume. In addition, the yellow area was continuously increased and the in this way identified $\beta_{1}-\beta_{2}$ combinations are presented in (c) as a function of their widths in the $\mathrm{y}$-and z-direction. 
As illustrated in figure 5.13 (a), the resolution in the $\mathrm{x}$ - and $\mathrm{y}$-direction behaves inversely to that in the $\mathrm{z}$-direction in terms of the $\beta_{1}-\beta_{2}$ combinations used for the reconstruction. The choice of low values for $\beta_{1}$ and $\beta_{2}$ lead to a higher resolution along the lateral directions, whereas the resolution in z-direction suffers. Higher $\beta_{1}$ and $\beta_{2}$ values result in a smaller width in z-direction. Therefore, a compromise is sought between good resolution in the lateral directions and in the z-direction. Furthermore, the volume of the effective PSF should be as small as possible. The volume of the effective PSF is proportional to the product of the FWHMs in the $\mathrm{x}, \mathrm{y}$ and $\mathrm{z}$ direction. Thus, the product of these FWHMs was calculated and investigated in relation to the FWHM in the ydirection. For this purpose, the effective PSFs of $\beta_{1^{-}} \beta_{2}$ combinations whose FWHM in $\mathrm{y}$-direction exhibits a value below a certain threshold were investigated. The procedure is illustrated in figure 5.13 (b), where the $\beta_{1}-\beta_{2}$ combinations considered accordingly, are marked in yellow. From these $\beta_{1}-\beta_{2}$ combinations, the combination was selected whose effective PSF exhibits the smallest volume. In addition, the yellow area was continuously increased and the in this way identified $\beta_{1^{-}} \beta_{2}$ combinations are presented in figure 5.13 (c) as a function of their widths in the $\mathrm{y}$ - and z-direction. Only the lateral width in the $\mathrm{y}$-direction is considered, since the FWHM in the $\mathrm{x}$-direction and in the $\mathrm{y}$-direction are approximately the same, as shown in figure 5.13 (a). The width in the z-direction of the selected combinations decreases, whereas the width in the y-direction increases. Moreover, the two curves intersect between their maximum and minimum values. The $\beta_{1}-\beta_{2}$ combination at the intersection point represents a compromise between the highest possible resolution in the y-direction, the best possible resolution in the z-direction and a minimum volume. Therefore, the combination at the intersection was chosen to be compared with conventional 3D STED in the following. For the case of the 19 orientations considered, $\beta_{1}$ was selected as $62^{\circ}$ and $\beta_{2}$ as $67^{\circ}$.

\subsection{D tomoSTED using the maximum-value reconstruction method}

To obtain high resolution information in all spatial directions, a final image has to be reconstructed from the individual 3D tomoSTED images. One reconstruction method, which was found to be suitable for tomoSTED in two dimensions, is the maximum-value reconstruction method (see section 3.3.1] [59, 60]. Therefore, the performance of 3D tomoSTED employing the maximum-value reconstruction was investigated by means of simulations regarding the question if at least an equal image quality can be achieved as 
the conventional 3D-STED while also using a lower light dose. The results are presented in the following. Equal image quality means an identical resolution in all spatial directions as well as the same total signal.

In order to obtain an impression of the performance of 3D tomoSTED, three exemplary arrangements of individual 3D tomoSTED PSFs with a low, medium and high (9, 19 and 153) number of orientations were investigated.23 For this purpose, the STED laser power of the individual 3D tomoSTED PSFs was reduced by the reduction factor of 2.61 derived in section 5.2 . Hence, a saturation factor of approximately 46 was used for simulating the individual 3D tomoSTED images, whereas an unscaled saturation factor of 120 was applied for the conventional 3D-STED.

The resolution within the maximum value reconstruction can be investigated using the effective PSF obtained by the maximum value reconstruction of the individual 3DtomoSTED PSFs (see sections 5.4 and 5.5). Cross sections of the obtained effective PSFs for the three arrangements in comparison to the PSF of the conventional 3D-STED are shown in figure 5.14 (a).

(a)

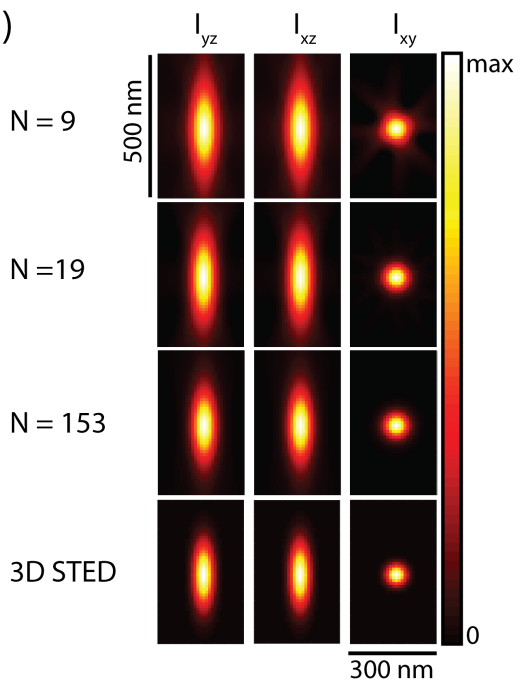

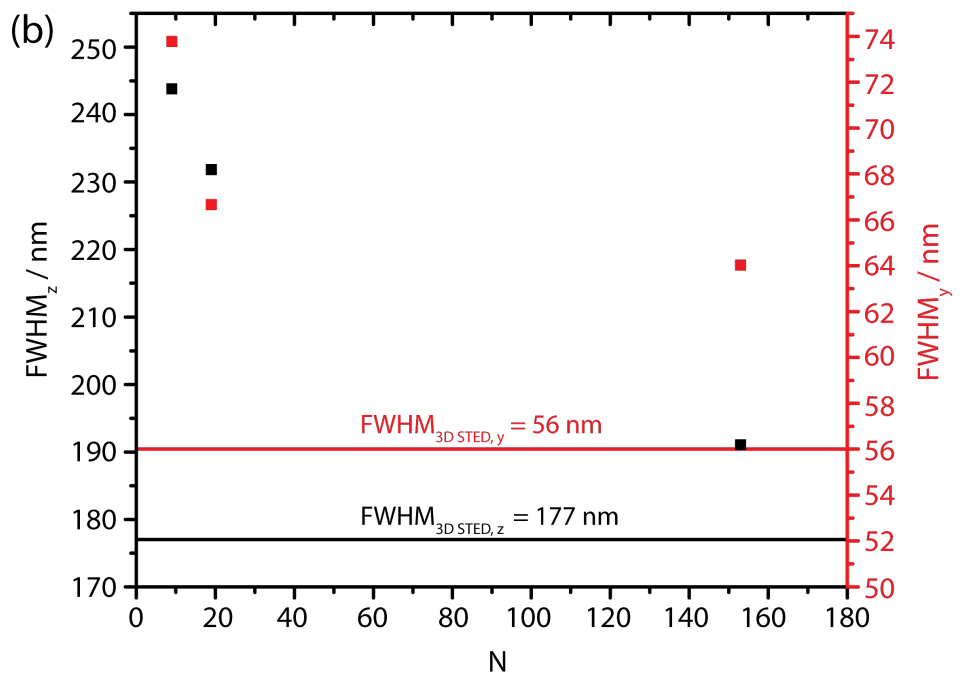

Figure 5.14: Comparison of final 3D tomoSTED PSFs reconstructed with 9, 19 and 153 orientations using the maximum-value reconstruction method with the conventional 3D STED PSF. (a) Cross-sections of the effective PSFs trough the y-z-, x-z-, and x-y plane. (b) Quantification of the resolution in $\mathrm{y}$ - and z-direction by means of FWHMs

Comparing the effective PSFs, it can be observed that their shape is quite similar. However, the reconstructed PSFs appear to be broader than the conventional one. Fur-

\footnotetext{
${ }^{23}$ For a more detailed description of the selection of the arrangement, please refer to section 5.4
} 
thermore, the broadening seems to decrease with increasing number of orientations. To quantify the resolution in more detail, the widths of these effective PSFs in terms of the FWHM were determined. For this, line profiles averaged over three neighbouring lines were drawn and the FWHM was determined. The result for the FWHMs in y- and $\mathrm{z}$-direction is presented in figure 5.14 (b). The FWHMs in $\mathrm{x}$-direction are shown in figure C.1 in section $C$ of the appendix. and exhibit similar values to those in the y-direction. The FWHM of the effective PSF reconstructed with 9 orientations is broader laterally by about $18 \mathrm{~nm}$ and axially by $67 \mathrm{~nm}$ than the conventional 3D STED. For the 19 orientations, the width is laterally wider by $11 \mathrm{~nm}$ and axially by $55 \mathrm{~nm}$ and for the 153 orientations, the difference in width amounts to $8 \mathrm{~nm}$ in lateral direction and to $14 \mathrm{~nm}$ in axial direction. In summary, the resolution of the conventional 3D STED could not be achieved for any of the considered cases.

Despite the lower resolution in all cases, the light dose is investigated for the three different types of arrangement. Considering light dose as the amount of irradiation on the sample applied by the STED beam, the strength can be characterised by the laser power employed to the sample and by the length of the exposure. The overall exposure time in 3D tomoSTED is given by the product of the exposure time for each individual orientation and the total number of orientations $\mathrm{N}$. The ratio between the light dose applied in 3D tomoSTED and the light dose employed in conventional 3D STED can thus be quantified as

$$
\mathrm{f}_{\text {light dose }}=\frac{1}{\nu} \frac{N}{\iota},
$$

where $\nu$ is defined as the reduction factor of the STED laser power and $\iota$ as the reduction factor of the exposure time. In order to obtain at least the same signal in all orientations as the conventional 3D STED, the exposure time of the individual PSFs can be reduced by the lowest signal ratio (see section 5.3. Therefore, ८ equals to 8.8. Using equation (102), the light dose of three considered 3D tomoSTED arrangements can be determined with respect to the conventional 3D STED and are listed below.

- $N=9: 0.39 \cdot$ light dose $_{\mathrm{cl}, 3 \mathrm{D}}$

- $N=19: 0.83 \cdot$ light dose $_{\text {cl, } 3 \mathrm{D}}$

- $N=153: 6.66 \cdot$ light dose $_{\mathrm{cl}, 3 \mathrm{D}}$

Another possibility, to achieve a higher resolution in the spatial directions, is to employ more STED laser power into the individual PSFs. Therefore, the arrangements of 9 and 
19 orientations were additionally investigated under lowering the laser power reduction factor $\nu$. For each $\nu$, an effective PSF was reconstructed and the respective FWHMs in lateral and axial direction were determined. The corresponding results for the 19 orientations are displayed in figure 5.15 and for the 9 orientation in figure C.2 in section Cof the appendix.

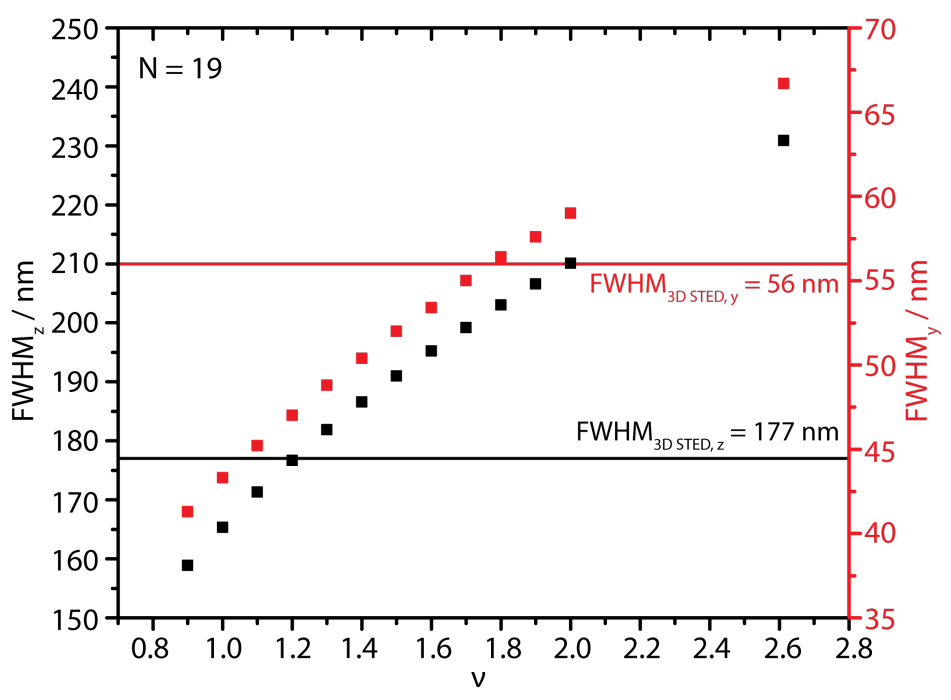

Figure 5.15: FWHM in y-and z-direction of the effective PSF reconstructed with 19 orientations as a function of the laser power reduction factor $\nu$ as compared to the conventional 3D-STED using a saturation factor of 120.

As illustrated in figures 5.15 and C.2, the arrangement of 19 orientations achieves approximately the same resolution as compared to the 3D STED at a factor $\nu$ of 1.2 and the 9 orientations at a $\nu$ value of 1 . Hence, a new light dose is calculated using these $\nu$ values. Moreover, the signal in the individual PSFs is decreased when the STED laser power is increased. Therefore, the exposure time reduction factor $\iota$ is redetermined. $\iota$ amounts to approximately 3.5 for the 19 orientations leading to a 4.5 times higher light dose than the conventional 3D STED. For the 9 orientations, $\iota$ was redetermined to be about 2.7, resulting in 3.3 times the light dose of 3D STED. Thus, although the both arrangements show approximately the same resolution as the conventional variant for the $\nu$ values of 1.2 and 1 , they exhibit a higher light dose than the conventional 3D STED. 24

\footnotetext{
${ }^{24}$ Additionally, the 3D tomoSTED arrangement of 9 number of orientations was investigating under lowering the laser power reduction factor which led as well to a higher light dose for 3D tomoSTED than for the conventional 3D STED. Moreover, an arrangement of 13 and 16 number of pattern orientations with two $\beta$-angles, as well as an arrangement of 20 and 27 number of pattern orientations with three $\beta$-angles were investigated, all of which featured a lower resolution when reducing the laser power by the factor of 2.61. Additionally, for none of the observed arrangements a lower light dose could be achieved when decreasing the laser power reduction factor.
} 


\subsection{D tomoSTED using Richardson-Lucy deconvolution}

Richardson-Lucy deconvolution has been demonstrated as a suitable reconstruction method for tomoSTED in two dimension [59, 60]. According to [59, 60], it allows to half the number of pattern orientations while still achieving the same image quality as the classical variant. With this, a light dose reduced by a factor of four could be achieved. Hence, [60] considered the Richardson-Lucy deconvolution as the preferred reconstruction method for tomoSTED. Thus, the performance of 3D tomoSTED via the Richardson-Lucy deconvolution is examined in the following via simulated data. It is investigated whether $3 \mathrm{D}$ tomoSTED shows at least the same resolving capability in all spatial directions as the conventional 3D STED while exhibiting at least the same signal. From this results, the expected light dose for 3D tomoSTED via the Richardson-Lucy deconvolution compared to the conventional $3 \mathrm{D}$ variant is discussed.

To exhibit at least the same image quality, 3D tomoSTED should at least resolve the same object as the conventional 3D STED. Thus, in the first step, the behaviour of the conventional 3D STED is examined which is presented in section 5.6.1. As described in section 3.3.2, the Richardson-Lucy deconvolution method iteratively estimates the object underlying the image. Thereby, the estimation procedure is limited by noise. Thus, the 3D STED is investigated at a certain signal to noise level. For this specific level, it is examined if the object is correctly estimated by the algorithm after the reconstruction. Here, the similarity between the estimate and the object is viewed at each iteration step in terms of normalised cross-correlation. Due to noise, which is additionally increased in each iteration step [15], the similarity increases until a certain number of iterations, where the estimate becomes dissimilar again (see section 3.3.2.4). Thus, the estimate with the highest normalised cross correlation is then compared to the object to see if it is a correct representation of the object. If this is the case, the object can be resolved by 3D STED. 3D STED exhibits laterally a higher resolution than axially. Thus, two ellipsoid shells were chosen as the object to probe the resolution in all spatial directions. By varying the diameters of the ellipsoid shells, it is investigated at which placed distances, the reconstruction method is still able to reconstruct the object correctly. The in this way obtained object is then in the following considered as the object which has to be as well correctly displayed by 3D tomoSTED in order to exhibit at least the same resolving performance. Thus, after having identified the object, it is examined whether $3 \mathrm{D}$ tomoSTED is as well capable to estimate the object correctly. The results 
are presented in section 5.6 .2 .

\subsubsection{Characterisation of the performance of classical 3D STED}

In a first step, the principal axes of the inner ellipsoid were chosen slightly larger than the 3D STED PSF's FWHMs. Thus, the diameter of the inner ellipsoid shell was set to $67 \mathrm{~nm}$ in lateral direction and to $200 \mathrm{~nm}$ in axial direction. The diameter of the outer shell was chosen as $207 \mathrm{~nm}$ in lateral direction and as $606 \mathrm{~nm}$ in axial direction. A y-zcross section of the object is presented in figure 5.16 (a).

To examine the influence of noise, data were simulated with and without noise, using a signal to noise ratio (SNR of 9 . In confocal microscopy, the noise can be modelled by Poisson noise [18, 62]. Thus, in the case of noise the data were simulated according to equation (103). ${ }^{25}$ where $\mathcal{P}$ represents the Poisson distribution [18, 62].

$$
I=\mathcal{P}(o * \mathrm{PSF})
$$

The data in the noise-free case were simulated only by the convolution between object o and PSF. Additionally, the background was assumed to be negligible. Thus, no background term was included into equation (103).

The similarity between the generated estimates and the object were mapped via the normalised cross-correlation. The normalised cross-correlation for the noiseless and noise containing case is shown in figure 5.16 (b). The curves follow the theoretical prediction. In the noiseless case, the similarity continuously increases and the normalised crosscorrelation converges to one. When considering the exemplarily represented line profile along the z-axis, it can be seen that the resolution increases per iterations step and if the number of iterations is high enough the estimate displays the object at its correct distances. Thus, in a noise free case the object can be recovered in any case.

In the considered case with noise, the similarity in terms of the normalised crosscorrelation increased until a value of approximately 0.94 and started then to decreases again. The maximum normalised cross-correlation was obtained at an iteration of 4000, corresponding to the highest similarity with the object. However, when observing the line-profile along the z-direction, it is noticeable that the object is not displayed correctly

${ }^{25}$ The Poisson noise was simulated applying the Matlab function poissrnd which randomly generates numbers from the Poisson distribution. 
(a)
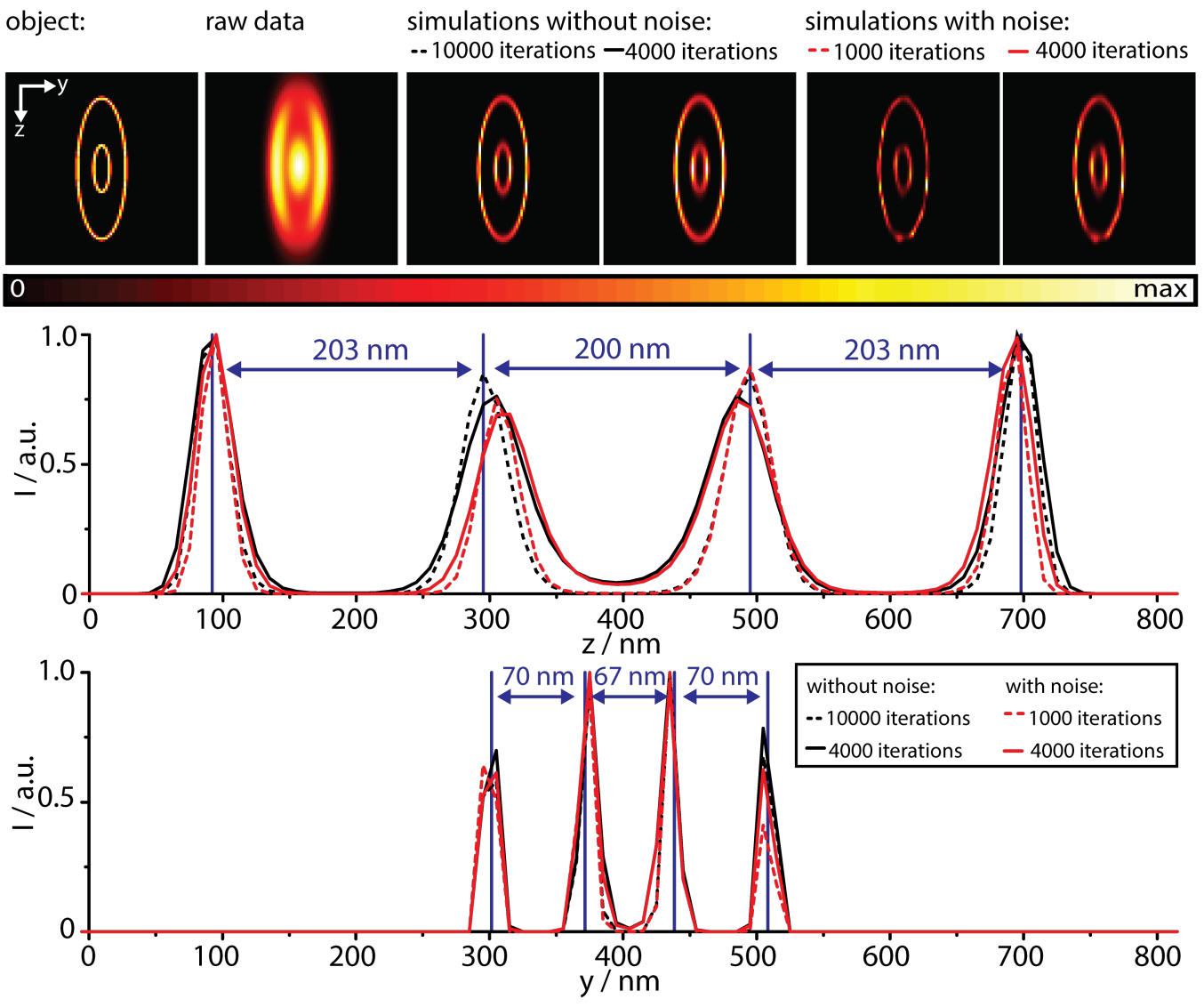

(b)

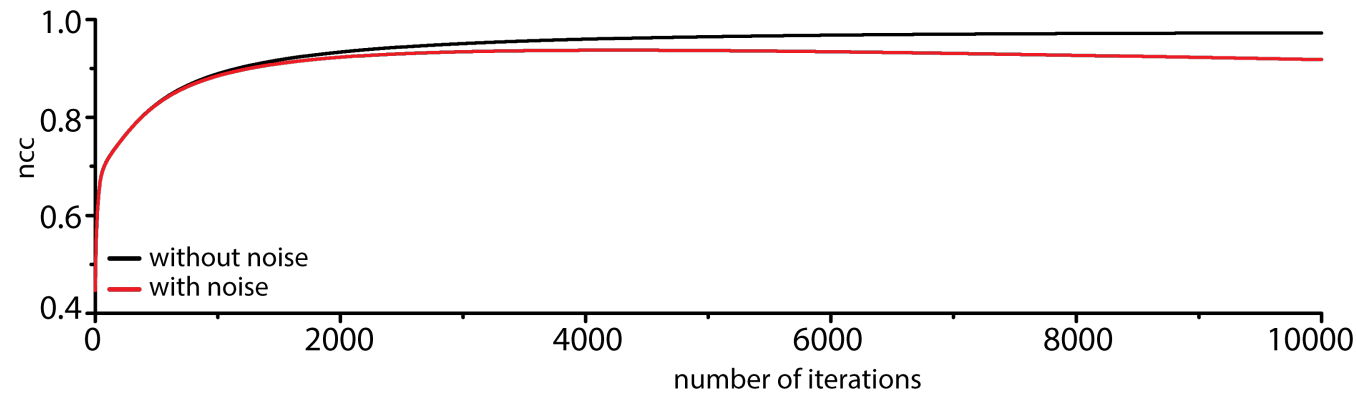

Figure 5.16: Investigation of the conventional 3D STED via the Richardson-Lucy deconvolution with and without noise. (a) Comparison of $y-z$ cross sections and line profiles along the z-axis of estimates obtained after 10000 iterations and 4000 iteration for the case with and without noise. (b) Maximum value of the normalised cross correlation as a function of the number of iterations for data simulated with and without noise

at this number of iterations. Hence, this number of iteration is not sufficient to recovery the object completely. At the SNR of 9 considered here, the distances between the ellipsoid shells are therefore too close to be fully recovered. Furthermore, the object is also not correctly represented at a higher iteration number in the case of noise present. In addition, the image quality degrades which agrees with the observations of the normalised cross-correlation. 
The choice of ellipsoid diameters in the previous section did not result in a spatially accurate estimate of the object. Therefore, the principle axis of the two ellipsoids were further increased and an object consisting of an inner ellipsoid shell with a diameter of $77 \mathrm{~nm}$ in lateral direction and of $210 \mathrm{~nm}$ in axial direction was investigated. The diameter of the outer ellipsoid shell was chosen to be $241 \mathrm{~nm}$ laterally and $640 \mathrm{~nm}$ axially. Using a SNR of about 9, the object could be fully recovered after the reconstruction procedure. The object, the estimates as well as line profiles in the y-and z-directions are presented in figure 5.17 .
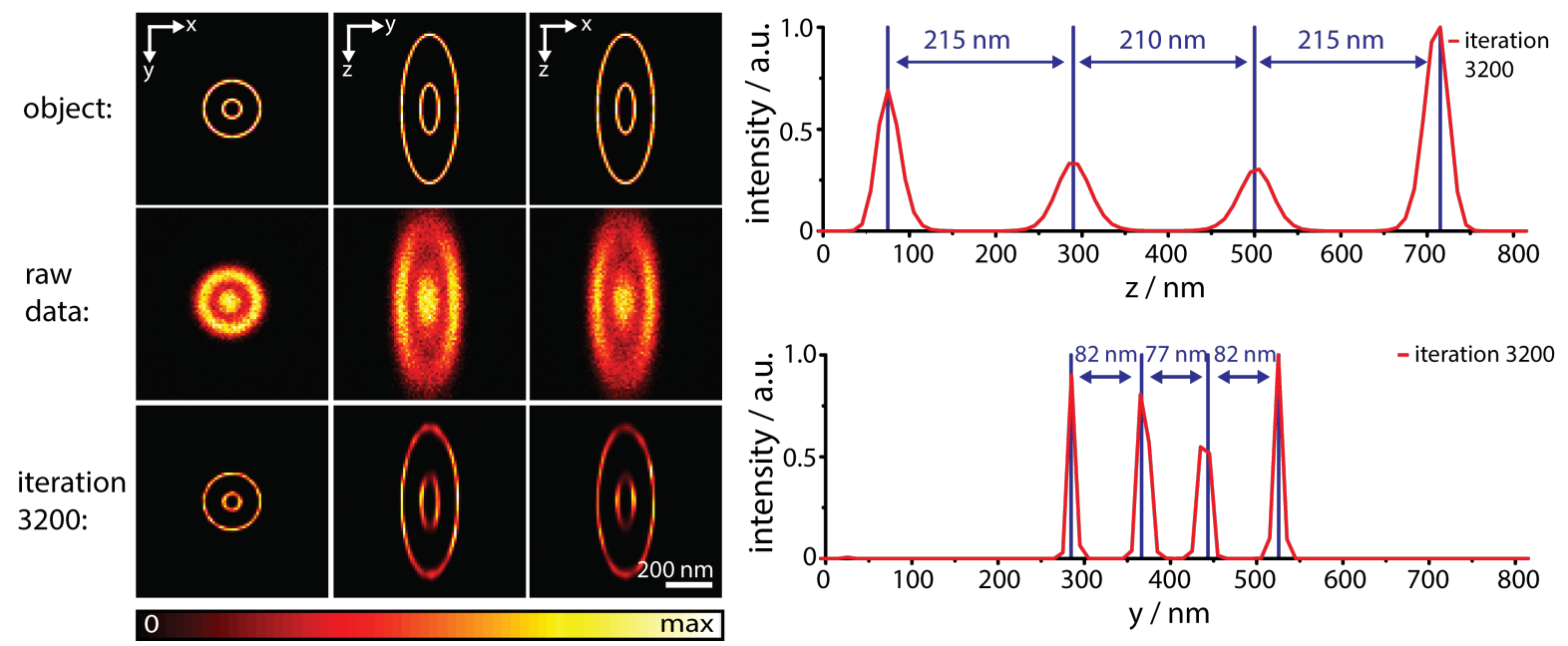

Figure 5.17: Illustration of the object identified as correctly recoverable for 3D STED using Richardson-Lucy deconvolution, in the form of cross-sections through the object, through the estimate with the highest similarity, and through the raw data containing noise. Exemplary line profiles along the y-and z-directions show that the distances of the object are preserved by the obtained estimate.

\subsubsection{Characterisation of the performance of 3D tomoSTED}

The object that has been identified as completely reconstructable by 3D STED (see section 5.6.1 was in the following examined by 3D tomoSTED. In order to show the same performance as 3D STED, the Richardson-Lucy deconvolution of 3D tomoSTED data should as well lead to estimates displaying this object's distances correctly. In a first step to investigate the performance of 3D tomoSTED via the Richardson-Lucy deconvolution, neither the laser power nor the signal was reduced compared to the performed characterisation of the conventional 3D STED. ${ }^{26}$ Therefore, all individual images ex-

\footnotetext{
${ }^{26}$ Thus, all individual tomoSTED images were calculated with the same saturation factor as used for simulating the conventional 3D STED (a saturation factor of 120).
} 
hibited a higher resolution as well as a higher signal. ${ }^{27}$ As for the characterisation of the conventional 3D STED (see section 5.6.1), the similartiy between the estimates and the object was mapped via the determination of the normalised cross-correlation. The estimates showing the maximum normalised cross-correlation were afterwards selected to be compared to 3D STED. Cross sections through the estimates for a 3D tomoSTED reconstruction with 9 and 19 pattern orientation are presented in figure 5.18, together with line profiles along the z-direction. To compare the performance with 3D STED, the line profile of the 3D STED reconstruction is additionally displayed in figure 5.18 .

3D tomoSTED: $\mathrm{N}=9$; iteration 24100:
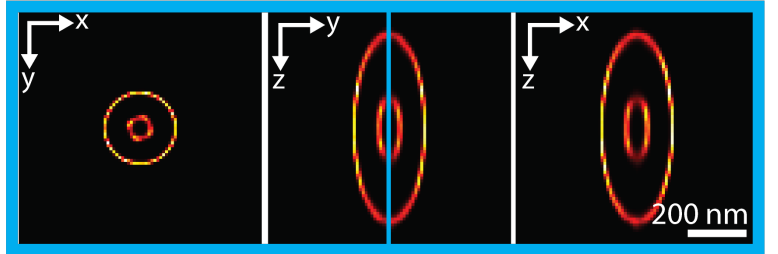

0
3D tomoSTED: $\mathrm{N}=19$; iteration 15600 :

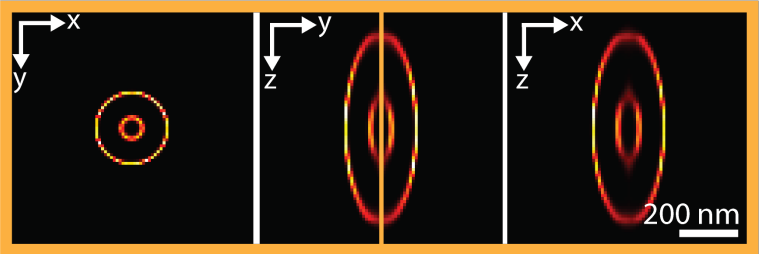

$\max$

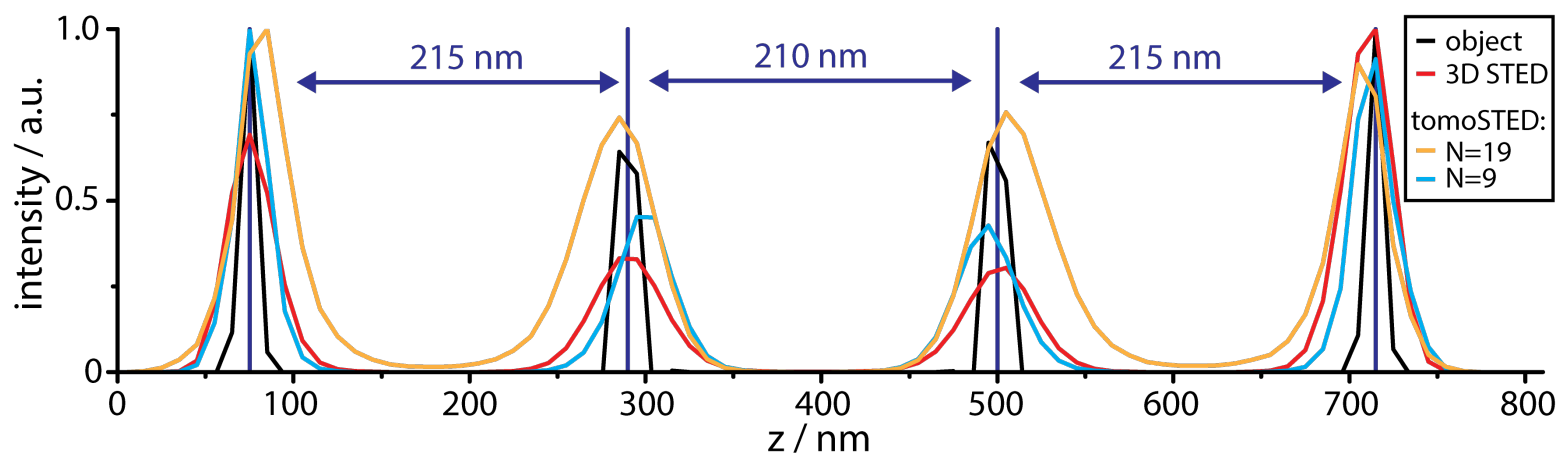

Figure 5.18: Reconstruction of 3D tomoSTED images with 9 pattern orientations and with 19 orientations via the Richardson-Lucy deconvolution with a saturation factor of 120 . Neither the laser power nor the signal was reduced for the reconstruction. Thus, the individual tomoSTED images exhibited a higher resolution than the conventional 3D STED as well as a higher signal. Line profiles along the z-direction show that the distances of the estimated ellipsoid shells deviates slightly for 3D tomoSTED from the distances of the underlying object.

3D tomoSTED requires a higher number of iterations to reach the point of maximum normalised cross-correlation than the classical 3D STED. An explanation for this observation might be that the information about the object for 3D tomoSTED is obtained by averaging the information from all individual orientations. Additionally, the individual tomoSTED images contain more low resolution content (more blur) that needs to be

${ }^{27}$ The lowest signal ratio was determined analogously to section 5.3 to 2.7 times the signal of the conventional 3D STED. 
got rid of. Thus, a higher number of iterations steps is required to reconstruct the object.

Comparing the line profiles in z-direction, it can be stated that the distances of the object in the reconstructed 3D tomoSTED data are not completely preserved. The distances for 3D tomoSTED deviate slightly by approximately $12 \mathrm{~nm}$. However, the intensities of the reconstruction results coincides better with the ground truth for 3D tomoSTED than for the conventional 3D STED. 


\section{Conclusion and Outlook}

The main objectives of this thesis were to investigate to what extend 2D tomoSTED can be improved with respect to sample gentleness, in terms of required light dose, by its combination with ISM, as well as the extension of the tomoSTED principle to threedimensional super-resolution imaging. A prerequisite for the experimental validation of these two objectives was the design and construction of a suitable microscope. This step was successfully accomplished by implementing the following modifications as compared to previous experimental realizations of tomoSTED [59, 97]: First, a sCMOS camera was utilized as a detector to enable array detection of the fluorescence signal in the vicinity of the excitation spot. Second, an additional SLM was added to provide another degree of freedom for STED phase mask generation. Third, the formerly used Pockels cells were replaced by LCRs to create arbitrary polarisation states of the STED beam without having to use high voltages. The characterization of the LCRs showed that all conceivable polarization states can be generated and that their switching time is comparable to those of the SLMs (see section 3.2.

Light dose reduction is achieved by reducing the number of highly resolved individual tomoSTED images along different directions which are required to achieve an isotropic resolution. Since this can only be accomplished if the resolution of the 1D STED-PSFs is increased in the diffraction-limited direction, tomoSTED was combined with ISM. Theoretical considerations as well as experiments proved that this enhances the resolution in the diffraction limited direction of the 1D STED-PSFs by about a factor of $\sqrt{2}$, while the resolution in the high-resolution direction is mainly maintained (see section 4.2). Furthermore, the number of required pattern orientations can be reduced by the same factor (see sections 4.3 and 4.4).

In future, the resolution in the diffraction-limited direction of the 1D STED-PSFs could be additionally improved by applying a Fourier filter during ISM pixel reassignment, as presented in [70]. This would allow for a further reduction of the number of required pattern orientations, leading to an even lower light dose. The pixel reassignment process could also be improved by using multi-image deconvolution, as demonstrated in [14]. Using a sCMOS camera for array detection is extremely slow, as the camera can be only read out with a maximum speed of $15 \mathrm{kHz}$. This was not a limiting factor for the comparison of ISM-tomoSTED with tomoSTED, since only fixed samples were used for validation. However, if living cells are to be observed, fast movements could lead 
to image artefacts. In this case, image acquisition can easily be accelerated to that of conventional STED microscopy by using a SPAD array [10, 14].

The second main objective of this thesis was the extension of the tomoSTED principle to three-dimensional super-resolution imaging. Therefore, it was first investigated theoretically which properties phase masks must possess to allow for STED resolution increase along an arbitrary spatial direction and how these can be adequately parametrized for a later experimental implementation. The derived class of phase masks consist of two disjoint regions exhibiting a phase delay of zero in one and $\pi$ in the other. The shape of the dividing line as well as its orientation in the $x-y$ plane determine the direction in which the high resolution is achieved. Expectedly, the phase masks known from literature for an optimal resolution enhancement in $\mathrm{x}-, \mathrm{y}$ - and $\mathrm{z}$-direction are included in this class. Measurements on gold microspheres proved that the depletion patterns, which correspond to this class of phase masks, can be generated in the experiment (see section 5.1).

In order to investigate whether the tomoSTED principle can be applied to three-dimensional super-resolution imaging by utilizing the beforehand identified depletion patterns for one-dimensional resolution enhancement, imaging was investigated by means of simulations for three exemplary arrangements (low, medium and high number) of pattern orientations. The arrangements with a low and a medium number of pattern orientations were optimized such that the achieved resolution represents a compromise between a high resolution in lateral as well as axial direction in such a way that a minimum focal volume is achieved (see section 5.4). When the individual tomoSTED images were reconstructed using the maximum value method, no light dose reduction can be achieved for any of the three arrangements while maintaining the image quality (see section 5.5). Nevertheless, parallel reconstruction of multiple individual 3D tomoSTED images using Richardson-Lucy deconvolution was analyzed. Since a reduction of the STED laser power and the exposure time is only reasonable if an approximately equal reconstruction result as for conventional 3D-STED microscopy can be achieved, the object reconstruction quality was investigated without any reduction of the STED laser power and the exposure time. It could be identified that the distances between the object structures in the deconvolved 3D STED data are preserved, while the deconvolved 3D tomoSTED data exhibit small deviations in the order of $12 \mathrm{~nm}$. However, the intensity distribution of the reconstructed object coincides better with the ground truth for 3D tomoSTED than for the conventional 3D STED (see section 5.6.). 
Based on the arrangements investigated in the context of this thesis, no possibility could be found to reduce the light dose required for high-resolution imaging by means of 3DtomoSTED. However, this investigation is not conclusive. Future research directions might be to explore other possibilities of arranging the class of 1D STED-PSFs identified in the context of this work. Since a high light dose is required in particular for 1D-PSFs whose high-resolution direction points clearly in the direction of the optical axis, and these are almost diffraction limited in the lateral direction, a significant reduction of the required light dose could possibly be achieved by their combination with ISM. Furthermore, the combination with ISM might also be advantageous with regard to aberration corrections, as estimating the shifts within the pixel reassignment enables aberration identification [56]. Moreover, this would have the advantage that the detector array used in ISM could be also employed to eliminate the out of focus signal present in the individual 1D STED-PSFs (see section 5.3), as demonstrated for STED microscopy by the authors of [69, 96. Another approach to 3D tomoSTED could also be to use an incoherent combination of two depletion patterns, e.g. the $1 \mathrm{D}$ patterns of $2 \mathrm{D}$ tomoSTED and the pattern of resolution increase in axial direction. This would transfer the light dose advantage of 2D tomoSTED to 3D imaging. Another possibility for improvement could be to explore other methods for the reconstruction of 3D tomoSTED data. One alternative could possibly be the use of a deep-learning algorithm [49, 100]. With sufficient training, such an algorithm might be able to generate a high-resolution image from a smaller number of orientations at an image quality which is comparable to that of the conventional 3D STED. In this context, however, it is also important to examine to what extent the reconstruction preserves distances between structures and their brightness, criteria, which have not been given much attention in the literature so far.

The 1D depletion patterns created by the derived class of phase masks, allow to obtain high resolution in any arbitrary spatial direction. Therefore, they could be used for applications beyond tomoSTED such as the targeted detection of biological structures, which allows for selective and therefore extremely fast and sample protective imaging. As concluded in [97], automatic selection of $1 \mathrm{D}$ depletion patterns according to the direction of the structure under investigation enables, in the ideal case, imaging to be restricted to the immediate vicinity of that structure and thus avoids imaging of areas that are of no or minor interest. 


\section{References}

[1] E. Abbe. Beiträge zur Theorie des Mikroskops und der mikroskopischen Wahrnehmung. Archiv für Mikroskopische Anatomie, 9:413-468, 1873.

[2] H. M. Adorf, R. N. Hook, L. B. Lucy, and F. D. Murtagh. Accelerating the Richardson-Lucy Restoration Algorithm. In European Southern Observatory Conference and Workshop Proceedings, volume 41 of European Southern Observatory Conference and Workshop Proceedings, page 99, 1992.

[3] Andor. Zyla sCMOSSpeed and Sensitivity for Physical Science Imaging and Spectroscopy. Technical report, Andor, opened May 2021. https://andor.oxinst.com/products/scmos-camera-series/zyla-4-2-scmos.

[4] T. Baur. Nematic Liquid Crystal Response Time. Meadowlark Optics Inc., 2008. Technical Note.

[5] M. Bertero, P. Brianzi, and E. Pike. Super-resolution in confocal scanning microscopy. Inverse Problems, 3:195-212, 1987.

[6] N. Bissantz, B. Mair, and A. Munk. A multi-scale stopping criterion for MLEM reconstructions in PET. 2006 IEEE Nuclear Science Symposium Conference Record, 6:3376-3379, 2006.

[7] M. Born and E. Wolf. Principles of Optics. Cambridge University Press, 7 edition, 1999.

[8] G. J. Brakenhoff, H. T. M. van der Voort, E. A. van Spronsen, and N. Nanninga. Three-Dimensional Imaging by Confocal Scanning Fluorescence Microscopy. Annals of the New York Academy of Sciences, 483(1):405-415, 1986.

[9] L. G. Brown. A Survey of Image Registration Techniques. ACM Computing Surveys, 24(4):325-376, 1992.

[10] M. Buttafava, F. Villa, M. Castello, G. Tortarolo, E. Conca, M. Sanzaro, S. Piazza, P. Bianchini, A. Diaspro, F. Zappa, G. Vicidomini, and A. Tosi. SPAD-based asynchronous-readout array detectors for image-scanning microscopy. Optica, 7 (7):755-765, 2020. 
[11] M. B. Cannell, A. McMorland, and C. Soeller. Image Enhancement by Deconvolution. In J. B. Pawley, editor, Handbook of Biological Confocal Microscopy, chapter 25, pages 488-500. Springer Science+Business Media, 2006.

[12] M. Castello, C. J. R. Sheppard, A. Diaspro, and G. Vicidomini. Image scanning microscopy with a quadrant detector. Optics Letters, 40(22):5355-5358, 2015.

[13] M. Castello, G. Tortarolo, M. Buttafava, T. Deguchi, F. Villa, S. Koho, P. Bianchini, C. J. R. Sheppard, A. Diaspro, A. Tosi, and G. Vicidomini. Image Scanning Microscopy with Single-Photon Detector Array. bioRxiv, 2018.

[14] M. Castello, G. Tortarolo, M. Buttafava, T. Deguchi, F. Villa, S. Koho, L. Pesce, M. Oneto, S. Pelicci, L. Lanzanó, P. Bianchini, C. J. R. Sheppard, A. Diaspro, A. Tosi, and G. Vicidomini. A robust and versatile platform for image scanning microscopy enabling super-resolution FLIM. Nature Methods, 16(2):175-178, 2019.

[15] J.-A. Conchello. Superresolution and convergence properties of the expectationmaximization algorithm for maximum-likelihood deconvolution of incoherent images. Journal of the Optical Society of America A, 15(10):2609-2619, 1998.

[16] J.-A. Conchello and J. G. McNally. Fast regularization technique for expectation maximization algorithm for optical sectioning microscopy. In C. J. Cogswell, G. S. Kino, and T. Wilson, editors, Three-Dimensional Microscopy: Image Acquisition and Processing III, volume 2655, pages 199 - 208. International Society for Optics and Photonics, SPIE, 1996.

[17] J. G. Danzl, S. C. Sidenstein, C. Gregor, N. T. Urban, P. Ilgen, S. Jakobs, and S. W. Hell. Coordinate-targeted fluorescence nanoscopy with multiple off states. Nature Photonics, 10(9):122-128, 2016.

[18] N. Dey, L. Blanc-Féraud, C. Zimmer, P. Roux, Z. Kam, and et al. 3D Microscopy Deconvolution using Richardson-Lucy Algorithm with Total Variation Regularization. RR-5272, INRIA.,pp.71, 2004. Research Report.

[19] A. Diaspro, G. Chirico, C. Usai, P. Ramoino, and J. Dobrucki. Photobleaching. In J. B. Pawley, editor, Handbook of Biological Confocal Microscopy, chapter 39, pages 690-701. Springer Science+Business Media, 2006.

[20] G. Donnert, J. Keller, R. Medda, M. A. Andrei, S. O. Rizzoli, R. Lührmann, R. Jahn, C. Eggeling, and S. W. Hell. Macromolecular-scale resolution in biological 
fluorescence microscopy. Proceedings of the National Academy of Sciences, 103(31): 11440-11445, 2006.

[21] M. Dyba and S. W. Hell. Focal Spots of Size $\lambda / 23$ Open Up Far-Field Florescence Microscopy at $33 \mathrm{~nm}$ Axial Resolution. Physical Review Letters, 88(16):163901, 2002.

[22] M. Dyba and S. W. Hell. Photostability of a fluorescent marker under pulsed excited-state depletion through stimulated emission. Applied Optics, 42(25):51235129, 2003.

[23] A. Egner, C. Geisler, C. von Middendorff, H. Bock, D. Wenzel, R. Medda, M. Andresen, A. C. Stiel, S. Jakobs, C. Eggeling, A. Schönle, and S. W. Hell. Fluorescence Nanoscopy in Whole Cells by Asynchronous Localization of Photoswitching Emitters. Biophysical Journal, 93(9):3285-3290, 2007.

[24] A. Egner, C. Geisler, and R. Siegmund. STED Nanoscopy. In T. Salditt, A. Egner, and D. R. Luke, editors, Nanoscale Photonic Imaging, volume 134 of Topics in Applied Physics. Springer Open, 2020.

[25] H. Foroosh, J. Zerubia, and M. Berthod. Extension of phase correlation to subpixel registration. IEEE Transactions on Image Processing, 11(3):188-200, 2002.

[26] C. Geisler, T. Hotz, A. Schönle, S. W. Hell, A. Munk, and A. Egner. Drift estimation for single marker switching based imaging schemes. Optics Express, 20(7): 7274-7289, 2012.

[27] F. Göttfert, T. Pleiner, J. Heine, V. Westphal, D. Görlich, S. J. Sahl, and S. W. Hell. Strong signal increase in STED fluorescence microscopy by imaging regions of subdiffraction extent. Proceedings of the National Academy of Sciences, 114(9): 2125-2130, 2017.

[28] M. Gu. Advanced Optical Imaging Theory. Springer-Verlag Berlin Heidelberg, 2000.

[29] M. Guizar-Sicairos, S. T. Thurman, and J. R. Fienup. Efficient subpixel image registration algorithms. Optics Letters, 33(2):156-158, 2008.

[30] M. Gustafsson, D. Agard, and J. Sedat. I5M: 3D widefield light microscopy with better than $100 \mathrm{~nm}$ axial resolution. Journal of Microscopy, 195(1):10-16, 1999. 
[31] M. G. Gustafsson, L. Shao, P. M. Carlton, C. J. R. Wang, I. N. Golubovskaya, W. Z. Cande, D. A. Agard, and J. W. Sedat. Three-Dimensional Resolution Doubling in Wide-Field Fluorescence Microscopy by Structured Illumination. Biophysical Journal, 94(12):4957 - 4970, 2008.

[32] M. G. L. Gustafsson. Nonlinear structured-illumination microscopy: Wide-field fluorescence imaging with theoretically unlimited resolution. Proceedings of the National Academy of Sciences, 102(37):13081-13086, 2005.

[33] B. Harke, J. Keller, C. K. Ullal, V. Westphal, A. Schönle, and S. W. Hell. Resolution scaling in STED microscopy. Optics Express, 16(6):4154-4162, 2008.

[34] B. Harke, C. K. Ullal, J. Keller, and S. W. Hell. Three-Dimensional Nanoscopy of Colloidal Crystals. Nano Letters, 8(5):1309-1313, 2008. PMID: 18166070.

[35] J. Heine, M. Reuss, B. Harke, E. D'Este, S. J. Sahl, and S. W. Hell. Adaptiveillumination STED nanoscopy. Proceedings of the National Academy of Sciences, 114(37):9797-9802, 2017.

[36] S. Hell and M. Kroug. Ground-state-depletion fluorscence microscopy: A concept for breaking the diffraction resolution limit. Applied Physics B, 60:495-497, 1995.

[37] S. Hell and E. H. K. Stelzer. Properties of a 4Pi confocal fluorescence microscope. Journal of the Optical Society of America A, 9(12):2159-2166, 1992.

[38] S. Hell, G. Reiner, C. Cremer, and E. H. K. Stelzer. Aberrations in confocal fluorescence microscopy induced by mismatches in refractive index. Journal of Microscopy, 169(3):391-405, 1993.

[39] S. W. Hell. Toward fluorescence nanoscopy. Nature Biotechnology, 21(11):1347$1355,2003$.

[40] S. W. Hell. Strategy for far-field optical imaging and writing without diffraction limit. Physics Letters A, 326(1-2):140-145, 2004.

[41] S. W. Hell. Far-Field Optical Nanoscopy. Science, 316(5828):1153-1158, 2007.

[42] S. W. Hell. Microscopy and its focal switch. Nature Methods, 6(1):24-32, 2009.

[43] S. W. Hell. Nanoscopy with focused light. Annalen der Physik, 527(7-8):423-445, 2015. 
[44] S. W. Hell and J. Wichmann. Breaking the diffraction resolution limit by stimulated emission: stimulated-emission-depletion fluorescence microscopy. Optics Letters, 19(11):780-782, 1994.

[45] S. W. Hell, M. Dyba, and S. Jakobs. Concepts for nanoscale resolution in fluorescence microscopy. Current Opinion in Neurobiology, 14(5):599-609, 2004.

[46] S. W. Hell, K. I. Willig, M. Dyba, S. Jakobs, L. Kastrup, and V. Westphal. Nanoscale Resolution with Focused Light: Stimulated Emission Depletion and Other Reversible Saturable Optical Fluorescence Transitions Microscopy Concepts. In Handbook Of Biological Confocal Microscopy, pages 571-579. Springer US, 2006.

[47] Z. Hrazdíra, M. Druckmüller, and S. Habbal. Iterative Phase Correlation Algorithm for High-precision Subpixel Image Registration. The Astrophysical Journal Supplement Series, 247(1):8, 2020.

[48] J. Huff. The Airyscan detector from ZEISS: confocal imaging with improved signalto-noise ratio and super-resolution. Nature Methods, 12:i-ii, 2015.

[49] M. Kawulok, P. Benecki, S. Piechaczek, K. Hrynczenko, D. Kostrzewa, and J. Nalepa. Deep Learning for Multiple-Image Super-Resolution. IEEE Geoscience and Remote Sensing Letters, 17(6):1062-1066, 2020.

[50] J. Keller. Optimal de-excitation patterns for RESOLFT-Microscopy. PhD thesis, Ruperto-Carola University of Heidelberg,, 2006.

[51] J. Keller, A. Schönle, and S. W. Hell. Efficient fluorescence inhibition patterns for RESOLFT microscopy. Optics Express, 15(6):3361-3371, 2007.

[52] I.-C. Khoo and S.-T. Wu. Optics and Nonlinear Optics of Liquid Crystals. WORLD SCIENTIFIC, 1993.

[53] T. A. Klar, S. Jakobs, M. Dyba, A. Egner, and S. W. Hell. Fluorescence microscopy with diffraction resolution barrier broken by stimulated emission. Proceedings of the National Academy of Sciences, 97(15):8206-8210, 2000.

[54] T. A. Klar, E. Engel, and S. W. Hell. Breaking Abbe's diffraction resolution limit in fluorescence microscopy with stimulated emission depletion beams of various shapes. Physical Review E, 64(6):066613, 2001. 
[55] A. Knigge, M. Schulz, C. Knothe, and U. Oechsner. Polarization analyzer for fiber optics and free beam applications. Technical report, Schäfter+Kirchhoff GmbH, 2021.

[56] S. Koho, F. Fersini, G. Tortarolo, M. J. Booth, and G. Vicidomini. Decoding Optical Aberrations in Laser Scanning Microscopy via a Detector-Array: Towards a New Adaptive Optics Architecture. In Focus on Mircoscopy 2021, Online, 2021.

[57] J. Kratz. Characterisation of Nanogel Particles Loaded with Proteins Employing Fluorescence-Based Techniques. Master's thesis, RWTH Aachen University, 2017.

[58] U. Krizic. Mulitple-View Microscopy with Light-Sheet Based Fluorescent Microscope. PhD thesis, University Heidelberg, 2009.

[59] J. Krüger. Tomographic STED Microscopy. PhD thesis, Georg August Universität Göttingen, 2017.

[60] J.-R. Krüger, J. Keller-Findeisen, C. Geisler, and A. Egner. Tomographic STED microscopy. Biomedical Optics Express, 11(6):3139-3163, 2020.

[61] C. D. Kuglin and D. C. Hines. The phase correlation image alignment method. Proc. Int'l Conf. Cybernetics and Society, 1975, pages 163-165, 1975.

[62] M. Laasmaa, M. Vendelin, and P. Peterson. Application of regularized Richardson-Lucy algorithm for deconvolution of confocal microscopy images. Journal of Microscopy, 243(2):124-140, 2011.

[63] J. R. Lakowicz. Principles of fluorescence spectroscopy. Springer, 3rd edition, 2006.

[64] J. W. Lichtman and J.-A. Conchello. Fluorescence microscopy. Nature Methods, 2 (12):910-919, 2005.

[65] L. B. Lucy. An iterative technique for the rectification of observed distributions. The Astronomical Journal, 79:745, 1974.

[66] L. B. Lucy and R. N. Hook. Co-adding Images with different PSF's. In D. M. Worrall, C. Biemesderfer, and J. Barnes, editors, Astronomical Data Analysis Software and Systems I, volume 25 of Astronomical Society of the Pacific Conference Series, page 277, 1992.

[67] J.-M. Masch. STED nanoscopy of synaptic substructures in living mice. PhD thesis, Georg-August-University Göttingen, 2017. 
[68] E. S. Meinel. Origins of linear and nonlinear recursive restoration algorithms. Journal of the Optical Society of America A, 3(6):787-799, 1986.

[69] M. Meschkat, M. G. Velasco, G. Schloetel, J. Rehman, F. Weidling, J. Heine, B. Thiel, C. A. Wurm, and M. Reuss. MATRIX STED - Many eyes see clearer: enhancing signal quality and brightness. In Focus on Mircoscopy 2021, Online, 2021.

[70] C. B. Müller and J. Enderlein. Image Scanning Microscopy. Physical Review Letters, 104(19):198101, 2010.

[71] B. Reddy and B. Chatterji. An FFT-based technique for translation, rotation, and scale-invariant image registration. IEEE Transactions on Image Processing, 5(8): 1266-1271, 1996.

[72] S. Remmele, B. Oehm, F. Staier, H. Eipel, C. Cremer, and J. Hesser. Reconstruction of high-resolution fluorescence microscopy images based on axial tomography. In B. M. Dawant and D. R. Haynor, editors, Medical Imaging 2011: Image Processing, volume 7962, pages 1468 - 1477. International Society for Optics and Photonics, SPIE, 2011.

[73] B. Richards, E. Wolf, and D. Gabor. Electromagnetic diffraction in optical systems, II. Structure of the image field in an aplanatic system. Proceedings of the Royal Society of London. Series A. Mathematical and Physical Sciences, 253(1274):358379, 1959.

[74] W. H. Richardson. Bayesian-Based Iterative Method of Image Restoration*. Journal of the Optical Society of America, 62(1):55-59, 1972.

[75] A. Rosenfeld and A. C. Kak. Digital Picture Processing (Second Edition), volume 2. Academic Press, 2 edition, 1982. p. 45-46.

[76] S. Roth, C. J. Sheppard, K. Wicker, and R. Heintzmann. Optical photon reassignment microscopy (OPRA). Optical Nanoscopy, 2(1):5, 2013.

[77] S. Roth, C. J. R. Sheppard, and R. Heintzmann. Superconcentration of light: circumventing the classical limit to achievable irradiance. Optics Letters, 41(9): 2109-2112, 2016.

[78] S. J. Sahl and S. W. Hell. High-Resolution 3D Light Microscopy with STED and RESOLFT, pages 3-32. Springer International Publishing, Cham, 2019. 
[79] S. J. Sahl, S. W. Hell, and S. Jakobs. Fluorescence nanoscopy in cell biology. Nature Reviews Molecular Cell Biology, 18(11):685-701, 2017.

[80] B. E. A. Saleh and M. C. Teich. Fundamentals of Photonics. John Wiley \& Sohn, Inc, 1991.

[81] K. Sätzler and R. Eils. Resolution improvement by 3-D reconstructions from tilted views in axial tomography and confocal theta microscopy. Bioimaging, 5(4):171$182,1997$.

[82] M. Sauer, J. Hofkens, and J. Enderlein. Handbook of Fluroescence Spectroscopy and Imaging. WILEY-VCH Verlag GmbH and Co, 2011.

[83] R. Schmidt, C. A. Wurm, S. Jakobs, J. Engelhardt, A. Egner, and S. W. Hell. Spherical nanosized focal spot unravels the interior of cells. Nature Methods, 5(6): 539-544, 2008.

[84] O. Schulz, C. Pieper, M. Clever, J. Pfaff, A. Ruhlandt, R. H. Kehlenbach, F. S. Wouters, J. Grosshans, G. Bunt, and J. Enderlein. Resolution doubling in fluorescence microscopy with confocal spinning-disk image scanning microscopy. Proceedings of the National Academy of Sciences, 110(52):21000-21005, 2013.

[85] P. J. Shaw. Comparison of Widefield/Deconvolution and Confocal Microscopy for Three- Dimensional Imaging. In J. B. Pawley, editor, Handbook of Biological Confocal Microscopy, chapter 23, pages 453-467. Springer Science+Business Media, 2006.

[86] L. A. Shepp and Y. Vardi. Maximum Likelihood Reconstruction for Emission Tomography. IEEE Transactions on Medical Imaging, 1(2):113-122, 1982.

[87] C. Sheppard. Super-resolution in confocal imaging. Optik - International Journal for Light and Electron Optics, 80:53, 1988.

[88] C. J. R. Sheppard. Pixel Reassignment in Image Scanning Microscopy. In K. Singh, A. K. Gupta, S. Khare, N. Dixit, and K. Pant, editors, ICOL-2019, pages 11-14, Singapore, 2021. Springer Singapore.

[89] C. J. R. Sheppard, X. Gan, M. Gu, and M. Roy. Signal-to-Noise Ratio in Confocal Microscopes. In J. B. Pawley, editor, Handbook of Biological Confocal Microscopy, chapter 22, page 442. Springer Science+Business Media, 2006. 
[90] C. J. R. Sheppard, S. B. Mehta, and R. Heintzmann. Superresolution by image scanning microscopy using pixel reassignment. Optics Letters, 38(15):2889-2892, 2013.

[91] C. J. R. Sheppard, M. Castello, G. Tortarolo, G. Vicidomini, and A. Diaspro. Image formation in image scanning microscopy, including the case of two-photon excitation. Journal of the Optical Society of America A, 34(8):1339-1350, 2017.

[92] C. J. R. Sheppard, M. Castello, G. Tortarolo, T. Deguchi, S. V. Koho, G. Vicidomini, and A. Diaspro. Pixel reassignment in image scanning microscopy: a re-evaluation. Journal of the Optical Society of America A, 37(1):154-162, 2020.

[93] C. J. R. Sheppard, M. Castello, G. Tortarolo, E. Slenders, T. Deguchi, S. V. Koho, G. Vicidomini, and A. Diaspro. Image scanning microscopy with multiphoton excitation or bessel beam illumination. Journal of the Optical Society of America A, 37(10):1639-1649, 2020.

[94] M. K. Singh, U. S. Tiwary, and Y.-H. Kim. An Adaptively Accelerated LucyRichardson Method for Image Deblurring. EURASIP Journal on Advances in Signal Processing, 2008(1):365021, 2007.

[95] T. Staudt, A. Engler, E. Rittweger, B. Harke, J. Engelhardt, and S. W. Hell. Far-field optical nanoscopy with reduced number of state transition cycles. Optics Express, 19(6):5644-5657, 2011.

[96] G. Tortarolo, S. Piazza, A. Bucci, P. Bianchini, C. J. Sheppard, A. Diaspro, E. Slenders, S. Koho, M. Castello, and G. Vicidomini. TIME-RESOLVED STED MICROSCOPY WITH SINGLE-PHOTON DETECTOR ARRAY: A PERFECT SYNERGY. In Focus on Mircoscopy 2021, Online, 2021.

[97] B. Vinçon. Adaptive Scanning for STED Microscopy. PhD thesis, Georg-August Universtität Göttingen, 2020.

[98] B. Vinçon, C. Geisler, and A. Egner. Pixel hopping enables fast STED nanoscopy at low light dose. Optics Express, 28(4):4516-4528, 2020.

[99] H. Wang, T. X. Wu, X. Zhu, and S.-T. Wu. Correlations between liquid crystal director reorientation and optical response time of a homeotropic cell. Journal of Applied Physics, 95(10):5502-5508, 2004. 
[100] J. Wang, J. Liang, J. Cheng, Y. Guo, and L. Zeng. Deep learning based image reconstruction algorithm for limited-angle translational computed tomography. PLOS ONE, 15(1):1-20, 2020.

[101] K. Weisshart. The Basic Principle of Airyscanning. Zeiss, 2014. Technology note.

[102] V. Westphal and S. W. Hell. Nanoscale Resolution in the Focal Plane of an Optical Microscope. Physical Review Letters, 94(14):143903, 2005.

[103] S. Wilhelm. Confocal Laser Scanning Microscopy. Technical report, Carl Zeiss Microscopy GmbH Jena, 2015.

[104] T. Wilson. Optical sectioning in fluorescence microscopy. Journal of Microscopy, 242(2):111-116, 2011.

[105] T. Wilson. Resolution and optical sectioning in the confocal microscope. Journal of Microscopy, 244(2):113-121, 2011.

[106] T. Wilson and C. Sheppard. Theory And Practice Of Scanning Optical Microscopy. London: Academic Press, -1, 1984.

[107] E. Wolf and D. Gabor. Electromagnetic diffraction in optical systems - I. An integral representation of the image field. Proceedings of the Royal Society of London. Series A. Mathematical and Physical Sciences, 253(1274):349-357, 1959.

[108] C. A. Wurm, K. Kolmakov, F. Göttfert, H. Ta, M. Bossi, H. Schill, S. Berning, S. Jakobs, G. Donnert, V. N. Belov, and S. W. Hell. Novel red fluorophores with superior performance in STED microscopy. Optical Nanoscopy, 1(1):7, 2012.

[109] R. Zanella, G. Zanghirati, R. Cavicchioli, L. Zanni, P. Boccacci, M. Bertero, and G. Vicidomini. Towards real-time image deconvolution: application to confocal and STED microscopy. Scientific Reports, 3(1):2523, 2013.

[110] B. Zhang, J. Zerubia, and J.-C. Olivo-Marin. Gaussian approximations of fluorescence microscope point-spread function models. Applied Optics, 46(10):1819-1829, 2007. 


\section{Appendices}

\section{A. Supplement to phase masks to STED microscopy}

To obtain a high resolution enhancement in a certain direction, the STED intensity distribution has to be as narrow as possible around the focal point along this direction. Thus, the steepness along the respective direction at the focal point has to be as maximal as possible. To get an idea of the behaviour of the steepness in the $\mathrm{x}-$, $\mathrm{y}$ - and $\mathrm{z}$-direction, the steepness is considered for these directions. The steepness is proportional the second derivative along the respective direction. This yields

$$
\left.\frac{\partial^{2} I(n)}{\partial n^{2}}\right|_{n=0} \propto 2 k^{2}\left(\int_{0}^{\alpha} \int_{0}^{2 \pi} P(\theta, \phi) \vec{c}(\theta, \phi) d_{n}(\theta, \phi) \sin (\theta) \mathrm{d} \theta \mathrm{d} \phi\right)^{2} \stackrel{!}{=} \max
$$

where $\mathrm{n}$ denominates the $\mathrm{x}-,-\mathrm{y}$, and $\mathrm{z}$-direction $(n=x, y, z)$. In analogy to section 5.1 . by neglecting the amplitude $\vec{c}(\theta, \phi)$, the steepness is determined by the contribution of the phase mask and additionally by the path length difference $d_{n}(\theta, \phi)$ :

$$
d_{\theta, \phi}= \begin{cases}-\sin (\theta) \cos (\phi) & \text { for } n=x \\ -\sin (\theta) \sin (\phi) & \text { for } n=y \\ \cos (\theta) & \text { for } n=z\end{cases}
$$

Equation 105 illustrates that $d(\theta, \phi)$ behaves complementary for the different directions. The complementary behaviour can also be seen in figure A.1, where $d(\theta, \phi)$ is illustrated with respect to the contributions from the aperture with aperture coordinates $\theta$ and $\phi$. 

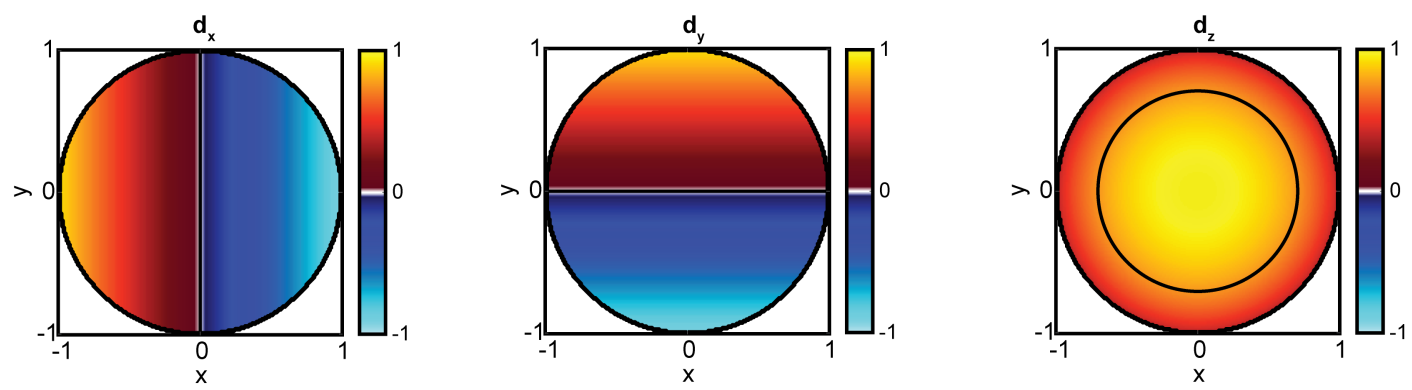

Figure A.1: Illustration of $d(\theta, \phi)$ for the $\mathrm{x}-, \mathrm{y}$ - and z-direction together with its median shown in black.

The highest steepness in each single direction would be obtained by applying a phase mask with the shape of the median (marked in black in figure A.1). However, this would result in a steepness of zero along the other directions. Thus, due to the complementary behaviour of $d(\theta, \phi)$ in the single directions, it is not possible to obtain a steep intensity distribution in all spatial directions. Furthermore, for a resolution enhancement in more than one direction by employing only one phase mask, a compromise between the different steepness behaviour has to be made resulting in a less steep intensity distribution. An example is the doughnut intensity distribution. The doughnut intensity distribution achieves an isotropic resolution enhancement in two dimensions. However, the steepness of the doughnut intensity distribution is lower than the one of the one-dimensional depletion pattern leading to a higher resolution enhancement for the one-dimensional case comparing the same laser power. 


\section{B. Supplement to validation of the pixel reassignment of ISM-tomoSTED via simulated data}
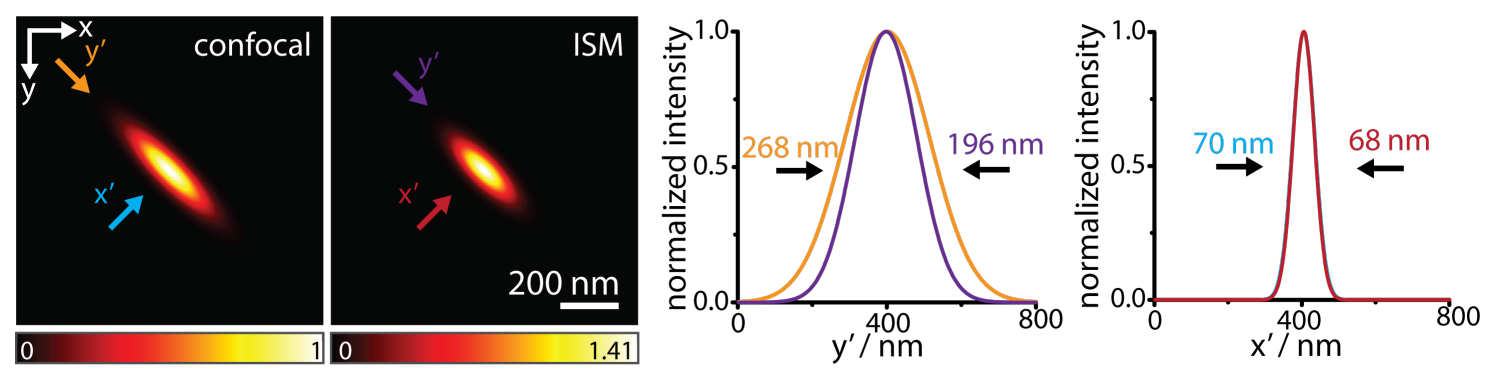

Figure B.1: Comparison of the $45^{\circ}$ tomoSTED and ISM-tomoSTED PSF including line profiles drawn along the diffraction-limited and high resolution direction 


\section{Supplement to 3D tomoSTED using the maximum-value reconstruction}

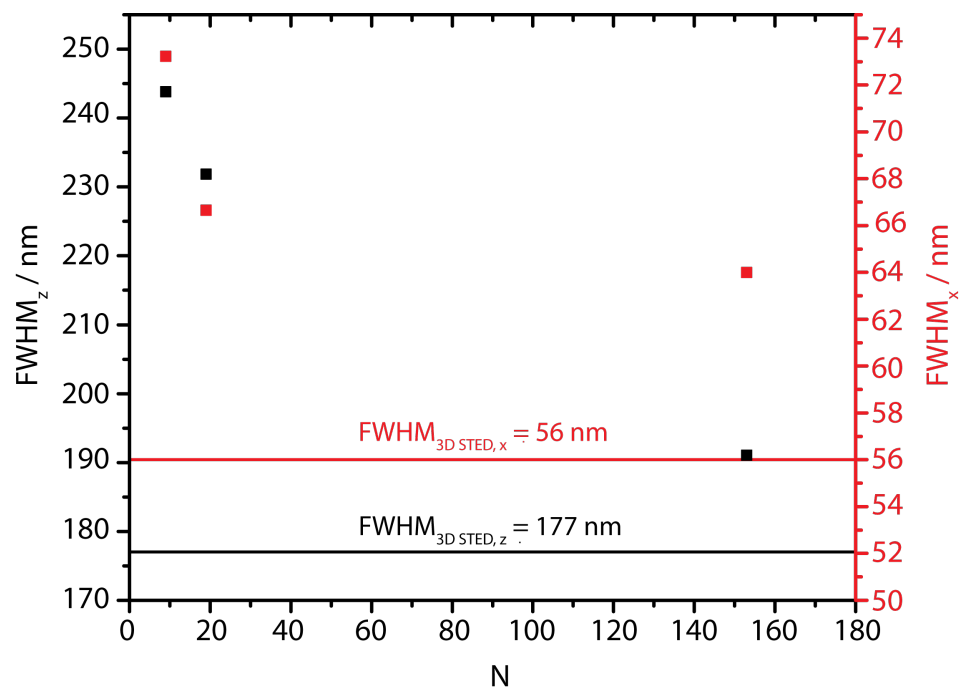

Figure C.1: Comparison of the resolution of the final 3D tomoSTED PSFs reconstructed with 9, 19 and 153 orientations using the maximum value reconstruction method with the resolution of conventional 3D STED. The resolution was quantified in terms of the FWHMs in the $\mathrm{x}$ - and z-directions, which was determined from line profiles drawn through the respective directions.

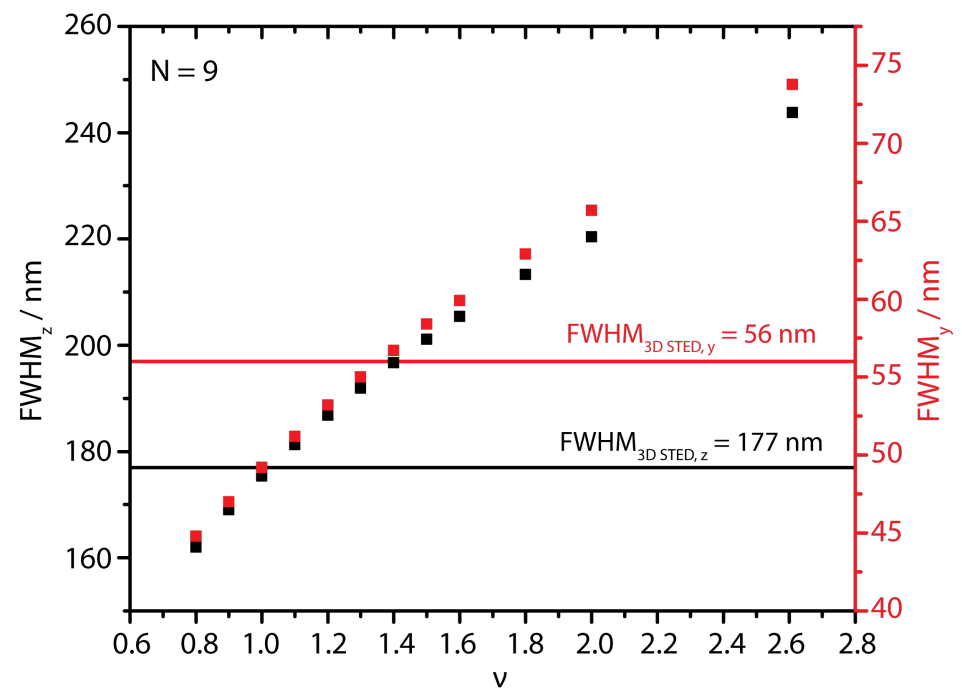

Figure C.2: Resolution in y-and z-direction of the effective PSF reconstructed with 9 orientations as a function of laser power reduction factor $\nu$ compared to the conventional $3 \mathrm{D}$ STED. 


\section{Acknowledgements}

After years of work and effort, it is time to acknowledge all the help and support. I would like to thank everyone who has contributed in different ways to the outcome of this thesis.

First of all, I would like to thank apl. Prof. Dr. Alexander Egner for giving me the opportunity to work on this thesis in his group and for supervision. I owe many thanks for his support in programming as well as the fruitful and constructive discussions.

Additionally, I thank Prof. Dr. Jörg Enderlein and Prof. Dr. Sarah Köster for acting as thesis advisors and for the constructive discussions during the advisory committee meetings. They have helped me to get one step further each time.

Furthermore, I owe thanks to Prof. Dr. Andreas Janshoff, apl. Prof. Dr. Michael Seibt, and Prof. Dr. Stefan Klumpp for spending the time and effort to complete my thesis committee.

Many special thanks go to Dr. Jan Keller-Findeisen for supporting my work by multiple fruitful discussions. In particular, I'm deeply grateful to him for his valuable contributions to the 3D phase masks and for proof-reading.

Moreover, I would like to thank all former and present members of the Optical Nanoscopy group for their useful help. In particular, I owe many thanks to Dr. Britta Vinçon and Dr. Francesco Rocca for their enriching help, the multiple discussions, their friendship as well as for proof-reading. Additionally, I would like to thank Dr. Kareem Soliman for providing me fixed cells and Dr. Claudia Geisler for her supervision.

In addition, I wish to express many thanks to all other colleagues at the IFNANO, especially the administration for a professional handling of all bureaucratic matters as well as the mechanical workshop for realising all adapters and mounting constructions. 


\section{Eidesstattliche Erklärung}

Hiermit erkläre ich, dass ich die vorliegende Arbeit selbstständig angefertigt, nicht anderweitig zu Prüfungszwecken vorgelegt und keine anderen als die angegebenen Hilfsmittel verwendet habe. Sämtliche wissentlich verwendeten Textausschnitte, Zitate oder Inhalte anderer Verfasser wurden ausdrücklich als solche gekennzeichnet.

Julia Kratz 